\title{
Understanding Cyclical Thrombocytopenia: A Mathematical Modeling Approach
}

\author{
Raluca Apostu
}

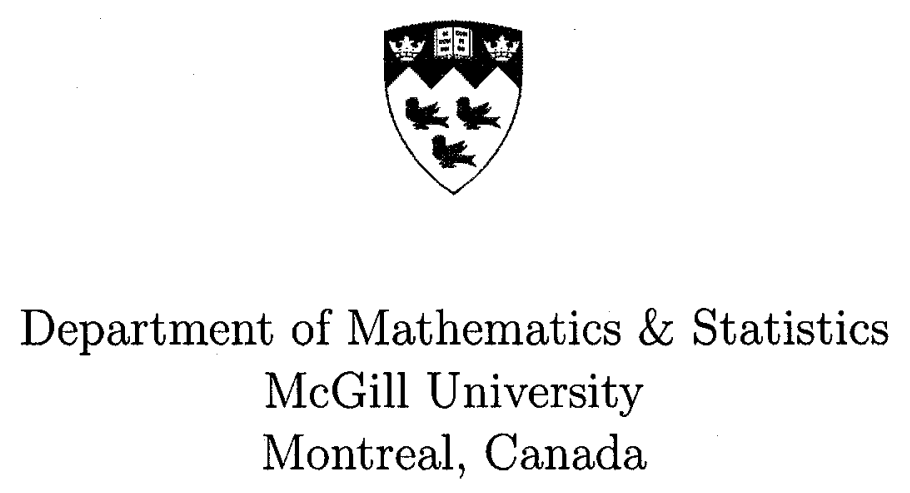

August 2007

A thesis submitted to McGill University in partial fulfilment of the requirements of the degree of Master of Science.

(C) 2007 Raluca Apostu 
Library and

Archives Canada

Published Heritage

Branch

395 Wellington Street

Ottawa ON K1A 0N4

Canada
Bibliothèque et

Archives Canada

Direction du

Patrimoine de l'édition

395 , rue Wellington

Ottawa ON K1A ON4

Canada

Your file Votre référence

ISBN: 978-0-494-38383-4

Our file Notre référence

ISBN: 978-0-494-38383-4

NOTICE:

The author has granted a nonexclusive license allowing Library and Archives Canada to reproduce, publish, archive, preserve, conserve, communicate to the public by telecommunication or on the Internet, loan, distribute and sell theses worldwide, for commercial or noncommercial purposes, in microform, paper, electronic and/or any other formats.

The author retains copyright ownership and moral rights in this thesis. Neither the thesis nor substantial extracts from it may be printed or otherwise reproduced without the author's permission.
AVIS:

L'auteur a accordé une licence non exclusive permettant à la Bibliothèque et Archives Canada de reproduire, publier, archiver, sauvegarder, conserver, transmettre au public par télécommunication ou par l'Internet, prêter, distribuer et vendre des thèses partout dans le monde, à des fins commerciales ou autres, sur support microforme, papier, électronique et/ou autres formats.

L'auteur conserve la propriété du droit d'auteur et des droits moraux qui protège cette thèse. $\mathrm{Ni}$ la thèse ni des extraits substantiels de celle-ci ne doivent être imprimés ou autrement reproduits sans son autorisation.
In compliance with the Canadian

Privacy Act some supporting forms may have been removed from this thesis.

While these forms may be included in the document page count, their removal does not represent any loss of content from the thesis.
Conformément à la loi canadienne sur la protection de la vie privée, quelques formulaires secondaires ont été enlevés de cette thèse.

Bien que ces formulaires aient inclus dans la pagination, il n'y aura aucun contenu manquant.

\section{Canada}




\section{Abstract}

Cyclical thrombocytopenia (CT) is a rare hematological disease characterized by periodic oscillations in the platelet count. Although first reported in 1936, the pathogenesis and an effective therapy remain to be identified. Since besides fluctuations in platelet levels the patients hematological profile have been consistently normal, a destabilization of a peripheral control mechanism might play an important role in the genesis of this disorder. In this thesis, we investigate through computer simulations the mechanisms underlying the platelet oscillations observed in CT. First, we collected the data published in the last 40 years and quantified the significance of the platelet fluctuations using Lomb-Scargle periodograms. Our analysis reveals that the incidence of the statistically significant periodic data is equally distributed in men and women. The mathematical model proposed in this thesis captures the essential features of hematopoiesis and successfully duplicates the characteristics of CT. With the same parameter changes, the model is able to fit the platelet counts and to qualitatively reproduce the TPO oscillations (when data is available). Our results indicate that a variation in the megakaryocyte maturity, a slower relative growth rate of megakaryocytes, as well as an increased random destruction of platelets are the critical elements generating the platelet oscillations in CT. 


\section{Résumé}

La thrombocytopénie cyclique (TC) est une rare maladie hématologique caracterisée par des oscillations périodiques dans les plaquettes sanguines. Bien qu'elle fût évoquée pour la première fois en 1936, la maladie et une thérapie efficace restent à trouver. Puisque malgré les fluctuations au niveau des plaquettes, les profiles hématologiques des patients restent toujour normaux, une destabilisation du méchanisme de contrôle périphérique peut jouer un rôle important dans la formation de ce maladie. Dans cette thèse, nous recherchons à travers des simulations informatiques les mechanismes sousjacent aux oscillations des plaquettes observées dans TC. En premier lieu, nous avons collecté les données publiées ces 40 dernière années et quantifié l'importance des fluctuations des plaquettes en utilisant les périodograms Lomb-Scargle. Notre analyse statistique révèle que les données périodiques sont équitablement distribuée chez les hommes et les femmes. Le modèle mathématique proposé dans cette thèse prend en compte les caractéristiques essentielles de la production des cellules sanguines et reproduit avec succès les charactéristiques de TC. Avec les même changement de parametèrs, le modèle reproduit bien le comportement des plaquettes sanguines et donne qualitativement les même oscillations que TPO (quand les données sont disponibles). Nos résultats indiquent que les éléments critiques générant les oscillations des plaquettes dans TC sont une variation dans la maturité du mégakaryocytes, un taux de croissance relativement lent des mégakaryocytes, ainsi que une augmentation aléatoire de destruction des plaquettes. 


\section{Acknowledgments}

I would like to thank my supervisor Dr. Michael Mackey for introducing me to this field and for bringing a new perspective to my life. I deeply appreciated his continuous guidance, support and inspiration.

I would also like to thank the following people: David Wolfson and Dima Jackobson for encouragement and advice in the first weeks in the master program, Moisés Santillán for suggestions in modeling the platelet dynamics, Caroline Colijn for help with the simulated annealing algorithm, Romain Yvinec for the French translation of the abstract, the friendly staff from Centre for Nonlinear Dynamics and Physiology Department, and all my friends for dragging me out from the office in Friday nights.

Most of all, I thank my parents for their love and support through the best and the worst days of this work.

Financial support for this research project was provided by MITACS (Mathematics of Information Technology and Complex Systems), and an NSERC (Natural Sciences and Engineering Research Council of Canada) grant to Dr. Michael Mackey. 


\section{Contents}

1 Introduction $\quad 1$

1.1 Hematopoiesis . . . . . . . . . . . . . . . . . . . 1

1.1.1 Blood cells development . . . . . . . . . . . . . 2

1.1.2 Platelet biogenesis ................. . . 3

1.1.3 Hematopoietic cytokines ............... 4

1.2 Cyclical thrombocytopenia . . . . . . . . . . . . 6

1.2.1 Cyclical thrombocytopenia as a dynamical disease . . . . . . 6

1.2 .2 Clinical profile. . . . . . . . . . . . . 6

1.2.3 Therapeutic attempts to treat cyclical thrombocytopenia . . . 8

1.2.4 Thrombocytopenia associated with other syndromes and as a side effect of chemotherapy . . . . . . . . . . . . 9

1.3 Organization of the thesis ................ 10

2 Spectral analysis of platelet data $\quad 11$

2.1 Lomb-Scargle periodogram . . . . . . . . . . . . . . . . 11

2.2 Results . . . . . . . . . . . . . . . . . . . . . 13

3 A mathematical model of hematopoiesis $\quad 25$

3.1 Differential delay equations . . . . . . . . . . . . . . 25

3.2 Model development . . . . . . . . . . . . . . . . . . . . 28

3.3 Parameter estimation . . . . . . . . . . . . . . . . . 35

4 Mathematical analysis of the model $\quad 38$

4.1 Model properties . . . . . . . . . . . . . . . 38 
4.2 Existence of steady states . . . . . . . . . . . . . . . 40

4.3 Linear stability analysis . . . . . . . . . . . . . . . . 43

4.3.1 Basic mathematical concepts . . . . . . . . . . . . . . 43

4.3.2 Linearized system and characteristic equation . . . . . . . 45

4.3.3 Subspaces of the stability region . . . . . . . . . . . . . 48

5 Simulating Cyclical Thrombocytopenia $\quad 63$

5.1 Parameters influence on the model dynamics . . . . . . . . . . . . 64

5.2 Simulated annealing method: description and advantages . . . . . . 68

5.3 Fitting the model simulations to the platelet data . . . . . . . . . . 71

5.4 Published TPO levels in cyclical thrombocytopenia patients . . . . . 79

5.5 Hypothesis for the origin of oscillations in cyclical thrombocytopenia 80

6 Conclusions $\quad 82$

6.1 Human TPO levels: megakaryocyte deficiency versus increased platelet destruction . . . . . . . . . . . . . . . . . 82

6.1.1 TPO kinetics in our model . . . . . . . . . . . . . . . . . . 84

6.1.2 Are the TPO levels exclusively dependent on the platelet number? 86

6.2 A short review . . . . . . . . . . . . . . 88

6.3 Discussion and future directions . . . . . . . . . . . . . . . 89

A Detailed calculations for the steady state(s) of the model (3.2.5) 91

B Predicted thrombopoietin levels in CT patients $\quad 94$

$\begin{array}{ll}\text { C XPP code } & 96\end{array}$

$\begin{array}{lr}\text { References } & 98\end{array}$ 


\section{List of Figures}

1.1 Hematopoietic regulation architecture . . . . . . . . . . . 2

1.2 Resting and activated platelets ............... 4

1.3 Bleeding symptoms in $\mathrm{CT} \ldots \ldots \ldots \ldots$

2.1 Drugs influence on platelet fluctuations . . . . . . . . . . . . . 14

2.2 Effect of various doses of prednisone on platelet cycles . . . . . . . . 15

2.3 Bar charts of the significant periods found in CT patients . . . . . . . 16

2.4 Data from Rice et al. (81) . . . . . . . . . . . . . . . 17

2.5 Published platelet counts of patients diagnosed with autoimmune CT 18

2.6 Published platelet counts of patients diagnosed with autoimmune CT 19

2.7 Published platelet counts of patients diagnosed with autoimmune CT

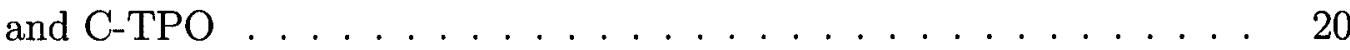

2.8 Published platelet counts of patients diagnosed with amegakaryocytic CT ............................... 21

2.9 Published platelet counts of patients diagnosed with amegakaryocytic $\mathrm{CT} \ldots \ldots \ldots \ldots \ldots 22$

2.10 Published platelet counts of patients diagnosed with amegakaryocytic

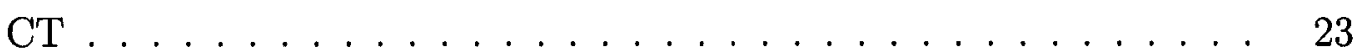

2.11 Published platelet counts of asymptomatic patients diagnosed with CT 24

3.1 The mathematic model of hematopoiesis . . . . . . . . . . . 29

4.1 Graphical argument for the existence and the uniqueness of the positive steady state . . . . . . . . . . . . . . . . . . 
4.2 Stability region bounded by the curves $\tau=\tau_{\text {crit }}$ and $A=-B . \quad \ldots \quad . \quad 57$

5.1 Highly sensitive model response to an increased $\gamma_{P} \ldots \ldots 4$

5.2 Evolution of periodicity in platelet oscillations when the maturation time of megakaryocytes $\tau_{P M}$ is modified. . . . . . . . . . . . . 65

5.3 Period and amplitude evolution when $\gamma_{P}$ and $\tau_{P M}$ are varied simultaneously . . . . . . . . . . . . . . . . . . . 66 66

5.4 Period evolution of platelet oscillations when $\tau_{P M}$ and $\mu$ are varied

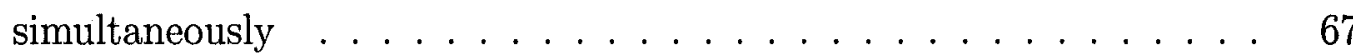

5.5 Period evolution of platelet oscillations when $\gamma_{P}$ and $\tau_{P M}$ are varied simultaneously

5.6 The significant periods found in reported cases of CT are linear combinations of the model delays. . . . . . . . . . . . . . . . . 68

5.7 3D representation of a typical energy landscape . . . . . . . . . 72

5.8 Fitting of CT data . . . . . . . . . . . . . . . 75

5.9 Fitting of CT data . . . . . . . . . . . . . . 76

5.10 Fitting of CT data . . . . . . . . . . . . . 77

5.11 Fitting of CT data . . . . . . . . . . . . . . . 78

5.12 Predicted and published TPO levels . . . . . . . . . . . . . 79

5.13 Photomicrographs of the bone marrow biopsy for an amegakaryocytic patient ....................... 81

6.1 TPO levels in patients with ITP and AA . . . . . . . . . . 83

6.2 TPO concentration as a function of platelet count . . . . . . . . . 84

6.3 TPO and platelet dynamics after a bolus intramuscular injection of thrombopoietin in healthy humans .............. 85

6.4 TPO response related to the megakaryocyte number rather than platelet counts. . . . . . . . . . . . . . . . . . . 87

B.1 Prediction of the thrombopoietin levels for amegakaryocytic patients . 94

B.2 Prediction of the thrombopoietin levels for patients diagnosed with C-TPO and autoimmune CT . . . . . . . . . . . . . . . . 995 


\section{List of Tables}

2.1 Inventory of the CT patients . . . . . . . . . . 17

3.1 Estimated equilibrium values for normal subjects. . . . . . . . . . 37

5.1 The significant periods found in cyclical thrombocytopenia patients are linear combinations of the model delays . . . . . . . . . . . . . . 69

5.2 Parameter estimates for the CT patients based on simulated annealing method. .................... 74 


\section{Chapter 1}

\section{Introduction}

This chapter presents the physiological framework of the research project and introduces the clinical features of cyclical thrombocytopenia (CT). CT is an unusual hematological disorder with dynamical character, and represents the object of our investigation.

\subsection{Hematopoiesis}

Over the last four decades available clinical data has allowed experimental hematologists to develop a model of hematopoiesis (blood cell production). Although the architecture of this process is continually evolving, the general outlines are already established. There is compelling evidence that all the mature blood cells are generated from a relatively small population of morphologically undifferentiated cells called hematopoietic stem cells (HSC). In humans, the HSC originate from the yolk sac in the first weeks of embryonic development. At about week six, the stem cells migrate to the substance of the embryo where they proliferate first in the fetal liver and next in the spleen. From about the fifteenth week of gestation through the death of the individual the bone marrow becomes the major site of hematopoiesis. In the marrow of a healthy person, most of the HSC are quiescent in the $G_{0}$ phase of the cell cycle and constitutes the reserve pool which is called into differentiation in the event of a hematopoietic stress. The stem cells have the capacity of self-renewal and differentiation. A small part of the total population from HSC is dedicated to peripheral blood 
cell formation and another fraction of it maintains the reserve pool through division (12).

\subsubsection{Blood cells development}

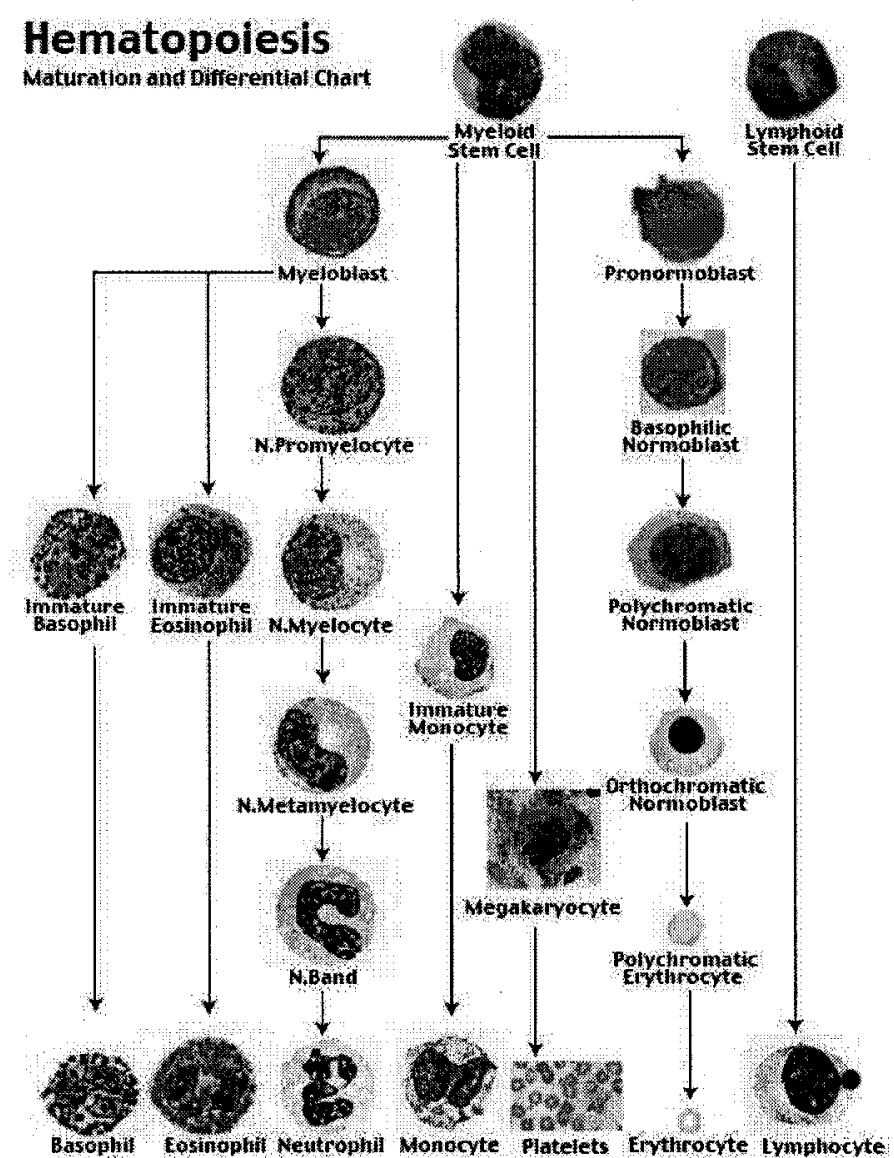

Fig. $\quad 1.1$ Hematopoietic regulation architecture (from http://www.userlogic.com/anll/studyaids/maturationchart/maturation_chart.html).

The differentiation of pluripotential stem cells is an hierarchical multi-step process involving many intermediate cell-types. The first step involves the commitment of the stem cells into one of the two major pathways, lymphoid or myeloid (Figure 1.1). The common lymphoid progenitors can generate only T, B, or NK lymphocytes, which take specific functions in the immune system. The myeloid lineage is 
more complicated. Three major branches can be distinguished: erythroid, megakaryocytic and phagocytic. The erythroid and megakaryocytic do not undergo further subdivision and give rise to platelets and respectively, red blood cells (RBC). The platelets have a major role in blood clotting and blood vessel repair, and $\mathrm{RBC}$ carry oxygen from the lungs to the tissues. The phagocytic pathway branches into monocytic and granulocytic lineages. The latter divides into neutrophilic, eosinophilic and basophilic lines. Neutrophils fight against bacterial infection, eosinophils destroy various parasites and reduce allergic inflammations. Basophils release histamine and serotonin, and modulate some inflammatory responses. Lymphocytes, neutrophils, monocytes, eosinophils, and basophils are constitutive parts of white blood cells (also called leukocytes).

\subsubsection{Platelet biogenesis}

Megakaryopoiesis incorporates the commitment of the pluripotential hematopoietic stem cells to the megakaryocytic differentiation, the proliferation, the maturation, and the terminal differentiation of the megakaryocyte progenitors. The megakaryocyte transition from immature cells to platelets starts with endoreduplication (duplication of the genome without mitosis), organelle synthesis, cytoplastic expansion, and formation of a microtubule array emanating from the centrosome (77). As the megakaryocyte matures, the nucleus becomes large and lobulated. DNA replication usually occurs at least three times during the complete sequence of endoreduplication to yield a mature megakaryocyte of ploidy 16 capable of platelet production (91). In addition to DNA and cytoplasmic expansion, an internal demarcation membrane, a dense tubular network, an open canalicular system, and a channeled system for granule release are formed (77). The internal demarcation membrane is connected to the plasma membrane and will become the external membrane of the proplatelets, the platelets precursors. The entire megakaryocyte cytoplasm is converted into a mass of proplatelets, the nucleus is extruded, and individual platelets are released from proplatelets ends (77).

Platelets (or thrombocytes), the smallest corpuscular components of the human blood ( $2-4 \mu m$ diameter), are important for blood coagulation and for haemosta- 
sis (arrest of bleeding). They adhere to the sites of damaged tissue to produce a hemostatic plug, and represent the surface on which the coagulation factors are activated to form a fibrin clot. The typical shape of resting platelets is discoid, but upon activation it changes to globular form with pseudopodia (up to $5 \mu \mathrm{m}$ long).
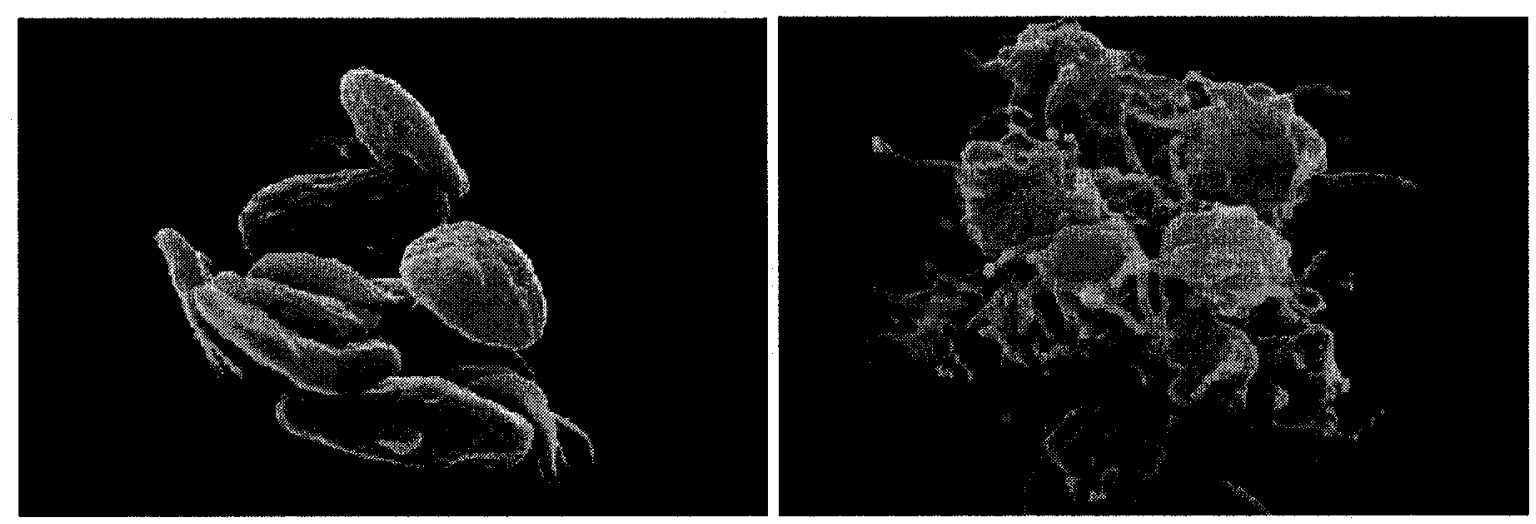

Fig. 1.2 Resting (left) and activated platelets (right). Platelets circulate in a dormant state. Endothelial damage in vivo and the absence of the vessel wall in in vitro experiments determine a platelet responsiveness to activating stimuli (2). Photos from http://www.perfusion.com/perfusion/articles/general/9905-plateletanatomy/

\subsubsection{Hematopoietic cytokines}

The proliferation of the stem cells and progenitor cells is controlled by a negative feedback system mediated by hematopoietic cytokines, which are hormones that regulate this process through endocrine and paracrine mechanisms.

Erythropoietin (EPO), which is the hormone that mediates the RBC production, is produced primarily in the kidney. A decrease in the number of $\mathrm{RBC}$ leads to lower oxygen levels in the tissue, which in turn trigger an increased production and release of EPO. Erythropoietin circulates in the blood to the bone marrow where it binds to the receptors on the surface of erythroid precursor cells, inducing their division and maturation. After a time delay due to division and maturation, a higher number of $\mathrm{RBC}$ are released into circulation, and the oxygen levels in the tissue increase accordingly. This cytokine has therapeutic applications: it is used to treat a variety 
of types of anemia.

Granulocyte colony stimulating factor (G-CSF) controls a similar negative feedback loop in the regulation of neutrophils. It has been shown that a decrease/increase in the circulating neutrophil counts leads to an increase/decrease in the production of G-CSF. G-CSF is widely used clinically to treat neutropenic patients (i.e. with low levels of neutrophils).

Thrombopoietin (TPO), known as C-Mpl ligand or megakaryocyte growth and development factor, is the primary regulator of thrombopoiesis (platelet production). Human TPO is encoded by a single gene, located on the chromosome 3 (q26.3-q27). Although TPO is produced constitutively by the liver, m-RNA specific for TPO is also found in the kidney, marrow stroma and other tissues (22). It was found experimentally that blood and marrow levels of TPO are inversely correlated to platelet count. This fact led to the hypothesis that thrombopoietin is mainly regulated by a consumption process in which TPO binds and activates the C-Mpl receptor on megakaryocytes and platelets, and then is removed from circulation $((32),(33),(60),(89))$. Although the understanding of platelet regulation is rapidly evolving, the molecular basis of this process is not completely elucidated. Substantial progress was achieved by the discovery and the cloning of TPO and its receptor in 1994 (49). This fact allowed the "simulation" of platelet formation in the laboratory for a better visualization and understanding of the process. It is believed that TPO stimulates hematopoietic stem cells to enter the cell cycle from their $G_{0}$ phase, accelerates proliferation and differentiation of megakaryocyte precursors, decreases precursor apoptosis, promotes megakaryocyte maturation, increases megakaryocyte ploidy and stimulates the release of platelets via the fragmentation of mature megakaryocytes (83). Moreover, TPO can act in synergy with other cytokines, and shares a high homology with EPO (22). Additionally, it plays an important role in myelopoiesis and erythropoiesis, acting on both lineage committed cells and on HSC (83). 


\subsection{Cyclical thrombocytopenia}

\subsubsection{Cyclical thrombocytopenia as a dynamical disease}

Hematopoiesis is a homeostatic system, and consequently most disorders of its regulation lead to chronic failures in the production of either all or only one blood cell type. Among the wide rage of diseases affecting the blood cells, there are some which are characterized by predictable oscillations in one or more cellular elements of the blood. They are called periodic or dynamical diseases (38). Cyclical neutropenia (CN), periodic chronic myelogenous leukemia (PCML), periodic auto-immune hemolytic anemia and cyclical thrombocytopenia (CT) are some classical examples. The investigation of their dynamic character offers an opportunity to enrich our knowledge about some regulating processes of blood cell production and has a clinical significance. The insights offered by a qualitative approach might help for a correct diagnosis and suggest therapeutic strategies.

In this work we focus on cyclical thrombocytopenia, specifically on its dynamic features. To provide a better understanding of the CT clinical profile, we present a comprehensive review of the literature and describe the experimental findings, treatment outcome, and current understanding of the pathophysiology.

\subsubsection{Clinical profile}

Cyclical thrombocytopenia is a rare hematological disorder described mostly in adults and characterized by periodic platelet count fluctuations of unknown etiology. It occurs predominantly in women and it was rarely observed in males. Sometimes this disease is associated with bleeding symptoms which have no apparent cause other than thrombocytopenia (low platelet count): purpura, petechiae, epitaxis, gingival bleeding, menorrhagia, easy bruising and gastrointestinal bleeding (see Figure 1.2.2).

For humans, the normal range of circulating platelets is $150 \times 10^{9}-450 \times 10^{9}$ platelets/L with an average of $290 \times 10^{9}$ platelets/L. Newborn babies have slightly a lower level, but they are usually within the adult range by the three months of age. Although, in general, human platelet levels remain relatively stable for years, many factors can influence an individual's platelet count (e.g. exercise, racial origin, 


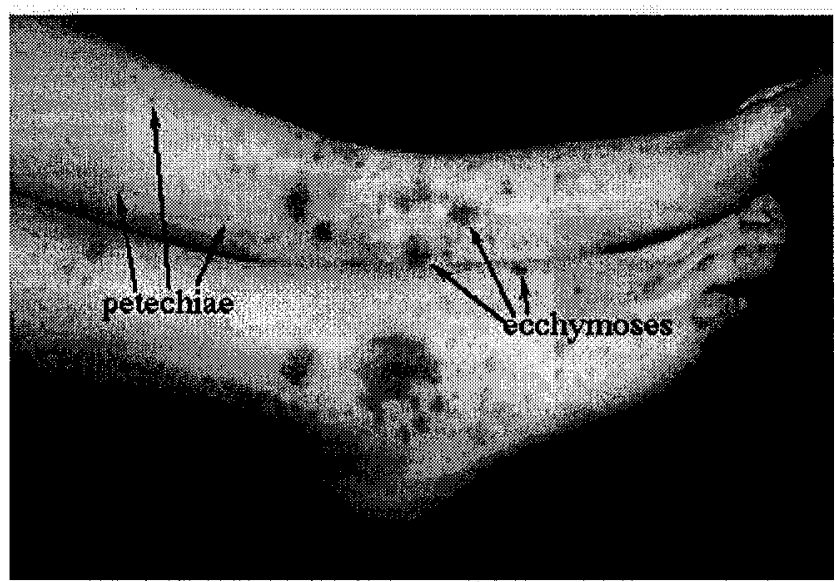

Fig. 1.3 Bleeding symptoms in CT

(from http://heme-coag.uthscsa.edu/wwwbleed97/00quantplt.html)

some diseases, pregnancy). In CT the platelet counts oscillate from very low $\left(1 \times 10^{9}\right.$ platelets/L) to normal or very high levels $\left(2000 \times 10^{9}\right.$ platelets/L). The literature contains 38 well-documented cases of platelet fluctuations (34 putative patients and 4 healthy individuals) published between 1962 and 2005. All these reports have been sporadic, except for an apparently unique family described by Aranda and Dorantes (3), in which the platelet cycling was observed in 4 out of 9 siblings and their father. Although CT is manifested mostly in adults with bleeding symptoms, our data collection includes the platelet count of two children $((15),(37))$ and three cases of asymptomatic individuals ((71), (92)).

The pathogenesis of CT is poorly understood and various mechanism have been proposed. The clinical findings suggest at least two pathways: immune-mediated platelet destruction (autoimmune cyclical thrombocytopenia) and megakaryocyte deficiency and cyclical failure in platelet production (amegakaryocytic cyclical thrombocytopenia). Autoimmune CT is thought to be an unusual form of immune thrombocytopenia purpura (ITP) and is more common in females. The hematological profile of most of these patients reveals high levels of antiplatelet antibodies, shorter platelet lifespan at the platelet nadir and normal to high levels of marrow megakaryocytes. Amegakaryocytic CT is postulated to be a variant of acquired amegakaryocytic throm- 
bocytopenic purpura and is mainly characterized by the absence of megakaryocytes in the thrombocytopenia phase and increased megakaryocyte number during thrombocytosis. Serial tests for serum antiplatelet antibodies are negative and the platelet lifespan is normal.

To determine the cause of cyclic megakaryocytopenia, Nagasawa et al. (74) examined the integrity of the megakaryocyte progenitor compartment just prior to the nadir and peak of platelet cycle. They noticed that in the autoimmune case the mean size of megakaryocytes does not change with the cyclic variations in the platelet count, while in patients with amegakaryocytic variety the number of colony-forming unitmegakaryocyte (CFU-Meg), the megakaryocyte number and the cytoplasmic area fluctuated in synchrony with the platelet cycle.

In all the reported cases, except for (36) and (70), besides oscillations in platelet count, the patient's hematological profile has been consistently normal. The peripheral red and white blood cell counts were within the normal range and the blood smear shows no morphological abnormalities or platelet clumps. In the case presented by Füreder et al. (36), erythropoiesis and granulopoiesis were slightly affected. The Menitove et al. (70) patient manifested a severe iron deficiency with anemia and reticulocytosis.

A synchronization between the fluctuations in the platelet count and menses has been reported in some female patients $((46),(72),(94))$. The fact that CT occurs also in men and women after menopause indicates that the pathogenesis of this disorder is not necessarily related to the menstrual cycle. For example, Cohen and Cooney (17) observe that platelet cycles are in phase with their patient's menstruation only when she was under exogenous hormone therapy, while other groups of investigators $((47),(70),(99))$ report no correlation between platelet oscillations and menses.

\subsubsection{Therapeutic attempts to treat cyclical thrombocytopenia}

Over time, various therapeutic measures have been tried. Since the general features of cyclical thrombocytopenia and immune thrombocytopenia purpura are very similar, many of the CT patients were misdiagnosed as ITP. Consequently, they received the typical ITP treatment like corticosteroids ((14), (15), (17), (29), (88), (95), (96)), in- 
travenous globulin ((4), (47), (56), (70), (93), (100)), plasma infusions (15), colchicine ((70), (81), (86)), and were subjected to splenectomy unnecessarily ((3), (4), (21), (46), (56), (58), (70), (81), (82), (86), (88), (93), (94), (96)). Despite their limitations, the case reports published in the literature suggest that traditional treatment appropriate for ITP does not have a benefic effect in CT cases (39). Up to now, no reliable therapy has been established, but danazol ((82), (94), (99)), cyclosporin A ((93), (100)), azathioprine (21), lynestrenol (95), glucocorticoids ((17), (29), (88), (94)) or hormonal contraception (46) have been shown to be effective in some cases.

\subsubsection{Thrombocytopenia associated with other syndromes and as a side effect of chemotherapy}

Although thrombocytopenia is common in HIV infection, it appears in association with other disorders, and it is an well-known side effect of chemotherapy, our analysis does not focus on these cases. When discussing about the causes and the management of cyclical thrombocytopenia it is important to distinguish between $\mathrm{CT}$ as an individual disorder and thrombocytopenia resulting from other causes. For completeness we present some examples.

Chemotherapy-induced thrombocytopenia is a problem oncologists have been fighting for years mostly because it represents one of the primary causes of morbidity and mortality in the treatment of cancer. It occurs because the chemotherapy drugs destroy rapidly growing cells, such as cells in the bone marrow that generate platelets. Due to the low number of circulating platelets, the chemotherapy treatments need to be delayed or dose-reduced, diminishing the patients chances for the most optimal result.

At the same time, the literature brings to attention cases of thrombocytopenia as a manifestation of myelodysplastic syndrome (79) or associated with other diseases like polycythemia vera (54), lymphatic-venous malformation (97) and Anaplasma spp. infection (39). 


\subsection{Organization of the thesis}

The first chapter presents the biological background of the project and introduces the clinical features of cyclical thrombocytopenia (CT). CT is a hematological disease with dynamic character and represents the object of our study. The first step in our investigation is the spectral data analysis of the platelet counts published in the literature (Chapter 2). Using periodogram analysis we test all data sets for the presence of statistically significant periodicity. To provide a better understanding of the mechanisms underlying the rhythmic fluctuations observed in this disorder, we employ a mathematical model of hematopoiesis. The model development and the parameter estimation based on clinical measurements are presented in Chapter 3. The model is consistent with the physiological framework and is mathematically correct. Chapter 4 tackles problems connected to the mathematical study of the model: the existence and the uniqueness of solutions (Section 4.1), the investigation of the steady states (Section 4.2), and the linear stability analysis (Section 4.3). The remainder of the thesis is original work. Our goal is to explain through computer simulations the onset of platelet oscillations which characterize CT. A detailed numerical analysis of the model dynamics allows us to zoom in the parameter space, and to identify the parameters with essential role in generating a model response similar with CT data (Section 5.1). The hematopoietic model successfully duplicates the platelet counts of CT patients, and provides a qualitative fitting of the thrombopoietin levels (when data available). In the case of some hematological disorders caused by either megakaryocyte deficiency or increased platelet destruction, the thrombopoietin levels are not accurately predicted by the circulating platelet counts. Since the understanding of the molecular basis of thrombopoiesis is not completely elucidated, we compare the TPO production in autoimmune CT and amegakaryocytic CT using the predictions of our model (Section 6.1). The biological interpretation of the results, the comparison with the clinical findings, and the conclusions drawn from the previous modeling effort are synthesized in the last chapter (Chapter 6). 


\section{Chapter 2}

\section{Spectral analysis of platelet data}

The focus of this chapter is to extract information about the oscillatory components of CT data. Searching the English literature from 1962 to 2006 we found 38 different clinical studies which report CT cases and publish the corresponding platelet counts. Although the data is presented either as cells $/ \mathrm{L}$ or as cells $/ \mathrm{kg}$ we maintain the same unit (cells $/ \mathrm{kg}$ ) and we use for conversion the fact that $70 \mathrm{~kg}$ adult has $6 \mathrm{~L}$ blood (18).

\subsection{Lomb-Scargle periodogram}

Many natural processes are periodic and the best way to describe this periodicity is through Fourier analysis. Since the experimental measurements cannot be fully controlled, the data sequence to be analyzed is often unevenly spaced in time and may contain random observational errors. Interpolating the unevenly sampled data to equally spaced time intervals and applying direct Fourier techniques may alter the perceived frequency and the significance of the periodic signal. In 1976, Lomb (65) proposed an extension of the Fourier power spectrum to deal with even and unevenly sampled data. His worked was continued a few years later by Scargle (85) who improved the periodogram and clarified the statistical assessment of a peak in the periodogram as a noise or signal.

Specifically, let $x_{j}$ be the concentration of the platelets as measured at time $t_{j}$, $j=\overline{1, N}$ where $N$ is the number of total data points. As usual, the mean and variance 
of data values are defined by

$$
\bar{x}=\frac{1}{N} \sum_{j=1}^{N} x_{j} \quad \text { and } \quad \sigma^{2}=\frac{1}{N-1} \sum_{j=1}^{N}\left(x_{j}-\bar{x}\right)^{2} .
$$

The Lomb-Scargle periodogram (also called Lomb normalized periodogram) defines the spectral power $P(\omega)$ as a function of the angular frequency $\omega=2 \pi f$ by the following formula:

$$
P(\omega)=\frac{1}{2 \sigma^{2}}\left\{\frac{\left(\sum_{j=1}^{N}\left(x_{j}-\bar{x}\right) \cos \omega\left(t_{j}-\rho\right)\right)^{2}}{\sum_{j=1}^{N} \cos ^{2} \omega\left(t_{j}-\rho\right)}+\frac{\left(\sum_{j=1}^{N}\left(x_{j}-\bar{x}\right) \sin \omega\left(t_{j}-\rho\right)\right)^{2}}{\sum_{j=1}^{N} \sin ^{2} \omega\left(t_{j}-\rho\right)}\right\}
$$

where $\rho$ is a constant defined implicitly by

$$
\tan (2 \omega \rho)=\frac{\sum_{j=1}^{N} \sin \left(2 \omega t_{j}\right)}{\sum_{j=1}^{N} \cos \left(2 \omega t_{j}\right)}
$$

The particular choice of $\rho$ has a double significance. It makes the periodogram invariant to time translations and equivalent to a least square fitting of sine curves to data. The second aspect partially explains the efficiency of the Lomb normalized periodogram with respect to Fourier transform methods: the data is weighted per point instead of per time interval. To determine the presence or absence of a periodic signal we need to quantify the significance of a peak in the periodogram. Consider the null hypothesis that the values $x_{j}$ are independent Gaussian random values. Scargle (85) normalized the Lomb periodogram by the total variance of the data $\sigma^{2}$. In this way, at any particular $\omega, P(\omega)$ has an exponential probability distribution with unit mean, i.e. the probability that $P(\omega)$ lies in a positive interval $(z, z+d z)$ is $e^{-z} d z$. Therefore, for $M$ independent frequencies, the probability that none give values larger than $z$ is $\left(1-e^{-z}\right)^{M}$. It follows that the false-alarm probability of the null hypothesis, i.e. the significance level $p$ of every peak we see in a periodogram, is given by

$$
p=1-\left(1-e^{-P(\omega)}\right)^{M}
$$


Some crucial ingredients in calculating the false alarm probability are the choice of the frequency range and the number $M$ of independent frequencies. Horne and Baliunas (48) performed numerical experiments for determining $M$ in different situations. In general, the number of independent frequencies depends on the number of data points $N$, their spacing and the number of frequencies sampled. Their numerical experiments showed that $M \approx N$ when the data points are approximately equally spaced (as in the platelet counts collected from the CT patients) or the sample frequencies oversample the frequency range. Press et al. (78) provide an effective way of computing $M$ under the assumption that there is no important clumping.

In determining the periodicity of the CT data we use a Matlab implementation of the algorithm proposed in Press et al. (78). In our work, an individual data set is considered periodic if the significance level $p$ of the principal peak in the periodogram is less than 0.05 .

Remark 1. 1. The presence of a significant peak at one of the frequencies implies that the data set is periodic with a period $P=\frac{2 \pi}{\omega}=\frac{1}{f}$.

2. Horne and Baliunas (48) prove that any other normalization of the Lomb periodogram except for the total variance of data will annihilate the definition of the false alarm probability given by (2.1.2).

3. For series sampled at constant time intervals, the Lomb periodogram yields the standard squared Fourier transformation.

\subsection{Results}

We found in the literature 38 case reports of patients diagnosed with cyclical thrombocytopenia. Using periodogram analysis we tested all data sets for the the presence of statistically significant periodicity in the platelet counts. Some of these studies presented patients without any treatment $((3),(64),(81),(98),(100))$, and others described subjects undergoing various therapies. Although most of the attempted therapeutic strategies were not successful, and the platelet counts continued to fluctuate, the type of treatment and the drug dose might influence the pattern of platelet 


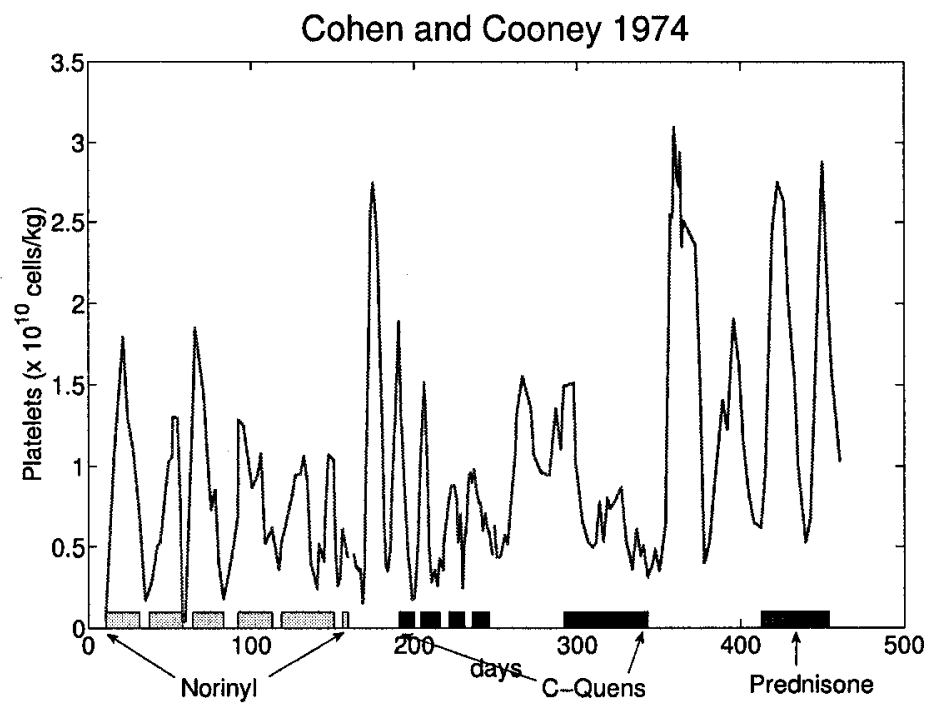

Fig. 2.1 Drugs influence on platelet fluctuations. Cohen and Cooney (17) observed the temporal evolution of the platelet levels for a young female undergoing various therapies (Norinyl, C-Quens, prednisone) as well as off treatment.

oscillations. For example, Cohen and Cooney (17) observed the temporal evolution of platelet levels for a young female over a period of 22 months. First she was treated with Norinyl, then with C-Quens, followed by prednisone (Figure 2.1). As in many other cases, prednisone did not alter the platelet cycles. The time series recorded during treatment with Norinyl or C-Quens display some important differences comparable to the off-therapy periods. The significant period, the amplitude and the mean of the platelet counts change according to the type of treatment. Lomb-Scargle periodogram reveals a period of 24.8 days (significance level 0.5 ) under Norinyl, which changes to 99 days (significance level 0.05) under C-Quens, and to 29 days (significance level 0.05) when the patient was off treatment. Although prednisone does not influence the platelet fluctuations, it was observed that various doses of it over a short time period can modify the oscillatory components of the time series. An example is the case of child with chronic thrombocytopenia purpura reported by Aranda and Dorantes (3). Analyzing the whole data set, regardless the dose of the drug, we find two significant periods ( 28 and 29 days, respectively). Under a constant dose of prednisone the platelet oscillations follow a regulated pattern with a significant period of 

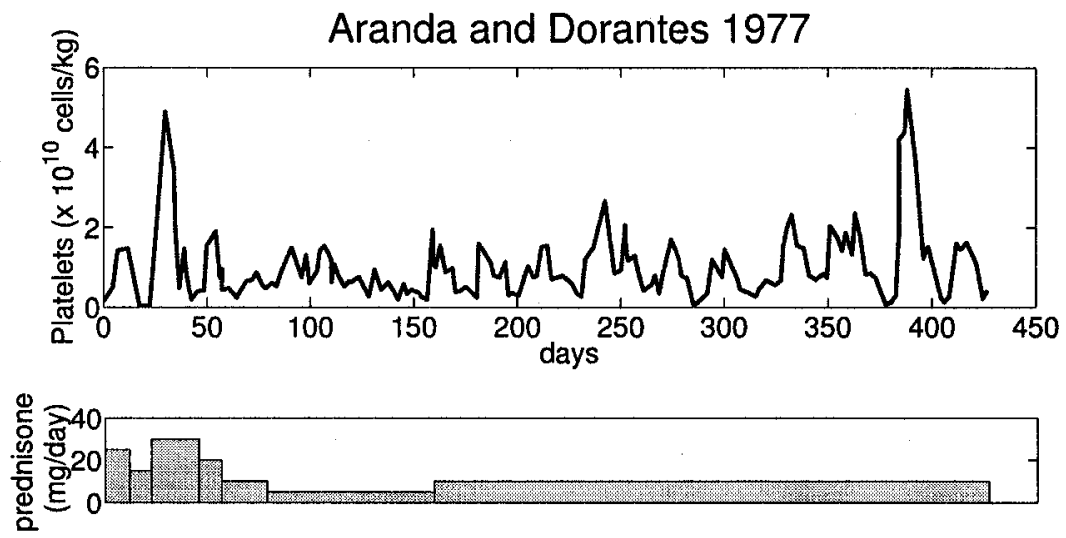

Fig. 2.2 Effect of various doses of prednisone on platelet cycles. The patient reported by Aranda and Dorantes (3) was treated with different doses of prednisone.

approximately 29 days (days 159-427 in Figure 2.2).

Given the variety of the data data published in the literature, we focus firstly on the time series corresponding to the patients without any treatment, secondly on the cases when the therapy does not alter the platelet cycles and, in the end, on the situations when the patient was undergoing different therapies. Our analysis is displayed graphically in Figures 2.5, 2.6, 2.7, 2.8, 2.9, 2.10, 2.11, where each data set is identified as to source. Based on our criterion for statistical significance $(p \leq 0.05)$, only 22 out of 38 data sets are significantly periodic (11 males and 11 females). The period of platelet oscillations varies between individuals, with a shorter average period in female ( $26 \pm 10$ days) comparable to men ( $35 \pm 12$ days). There is a connection between the patient's diagnosis and the range of significant periods of platelet variations. The oscillations in autoimmune data have periods ranging from 13 to 31 days which, on average, are shorter than the periods in amegakaryocytic cases. The latter vary from 19 to 64 days (Figure 2.3). Table 2.1 summarizes the results of our data analysis. For each patient we specify the sex, the diagnosis, and the significant period(s). 

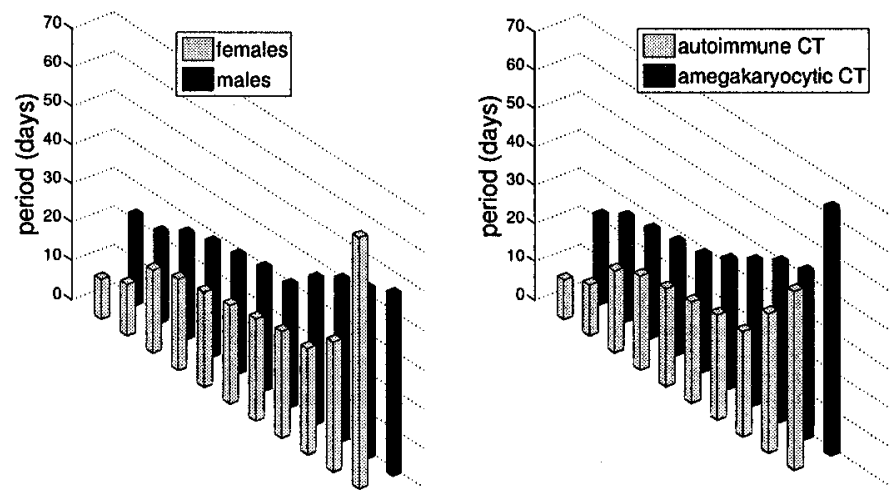

Fig. 2.3 Bar charts of the significant periods found in cyclical thrombocytopenia patients. Shorter oscillation periods in autoimmune CT comparable to amegakaryocytic CT. Similar situation for female versus male cases.

Remark 2. To help in deciphering the pattern of oscillations observed in $C T$, we search for other characteristic elements of the time series. Once a significant periodicity $P$ has been detected through periodogram analysis, we can easily estimate the phase $f$ and amplitude $A$ of a sine wave that best fits the data using the function

$$
X_{j}=A \sin \left(\frac{2 \pi t_{j}}{P}+f\right), \quad j=\overline{1, N}
$$

If the time series has two significant periods $P_{1}$ and $P_{2}$ then we need two sine waves:

$$
X_{j}=A_{1} \sin \left(\frac{2 \pi t_{j}}{P_{1}}+f_{1}\right)+A_{2} \sin \left(\frac{2 \pi t_{j}}{P_{2}}+f_{2}\right), \quad j=\overline{1, N}
$$

There is no apparent relationship between the mean of data, the period and the amplitude of oscillations in the platelet compartment. 


\begin{tabular}{|c|c|c|c|c|}
\hline Source and patient ID & Sex & Diagnosis & $\begin{array}{l}\text { Mean of the data } \\
\left(\times 10^{10} \text { cells } / \mathrm{kg}\right)\end{array}$ & $\begin{array}{c}\text { Significant } \\
\text { period(s) } \\
\text { (days) }\end{array}$ \\
\hline Fogarty et al. (2005) & $\bar{M}$ & $\bar{A} \mathbf{I}$ & 1.7741 & 46 \\
\hline Rice et al. (2001) & $\mathrm{F}$ & AI\&AM & 0.1042 & 27 \\
\hline Kimura et al. (1996) & M & AI\&AM & 1.579 & 37 and 9 \\
\hline Helleberg et al. (1995) & $\mathrm{F}$ & AI & 0.9059 & 27 \\
\hline Kosugi et al. (1994) & $\mathrm{F}$ & AI & 0.3290 & 25 \\
\hline Yanabu et al. (1993) & $\mathrm{F}$ & AI & 1.9507 & 24 \\
\hline Rocha et al. (1991) & $\mathrm{F}$ & AI & 1.0455 & 21 and 10 \\
\hline Menitove et al. (1989) & $\mathrm{F}$ & AI & 1.1452 & 13 \\
\hline Skoog et al. (1957) & $\mathrm{F}$ & AI & 0.5625 & 26 \\
\hline Bruin et al. (2005) & $\mathrm{M}$ & AM & 0.6304 & 27 \\
\hline Füreder et al. (2002) & $\mathrm{F}$ & $\mathrm{AM}$ & 1.7806 & 27 \\
\hline Zent et al. (1999) & $\mathrm{M}$ & $\mathrm{AM}$ & 0.7267 & 30 \\
\hline Hoffman et al. (1989) & $\mathrm{F}$ & AM & 1.1260 & 64 \\
\hline Aranda and Dorantes (1977) & $\mathrm{M}$ & AM & 1.0984 & 29 \\
\hline Cohen and Cooney (1974) & $\mathrm{F}$ & $\mathrm{AM}$ & 0.8064 & 30 \\
\hline Wilkinson and Firkin (1966) & $\mathrm{M}$ & $\mathrm{AM}$ & 1.8701 & 41 \\
\hline Wasastjerna (1967) & $\mathrm{M}$ & AM & 1.1354 & 23 \\
\hline Engstrom et al. (1966) & $\mathrm{M}$ & AM & 1.7984 & 43 \\
\hline von Schulthess and Gesser (1986), case 1 & $\mathrm{M}$ & Healthy & 1.9858 & 23 \\
\hline von Schulthess and Gesser (1986), case 2 & M & Healthy & 2.9230 & 31 and 49 \\
\hline Morley (1969), subject 8 & $\mathrm{M}$ & Healthy & 1.7704 & 31 \\
\hline Lewis (1974) & $\mathrm{F}$ & C-TPO & 0.9180 & 23 \\
\hline
\end{tabular}

Table 2.1 Inventory of the CT patients. AI indicates autoimmune CT, AM denotes amegakaryocytic CT diagnosis, C-TPO stands for cycling TPO levels, and "healthy" are the asymptomatic patients.

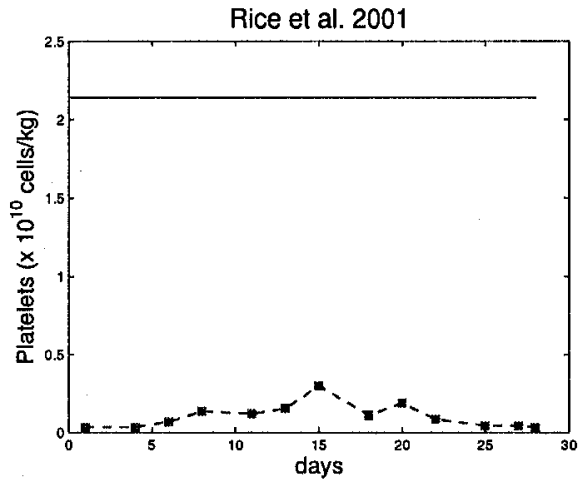

Fig. 2.4 The patient reported by Rice et al. (81) was responsive to thrombopoietic growth factor therapy. In our investigation we use the data collected one cycle prior to treatment. 

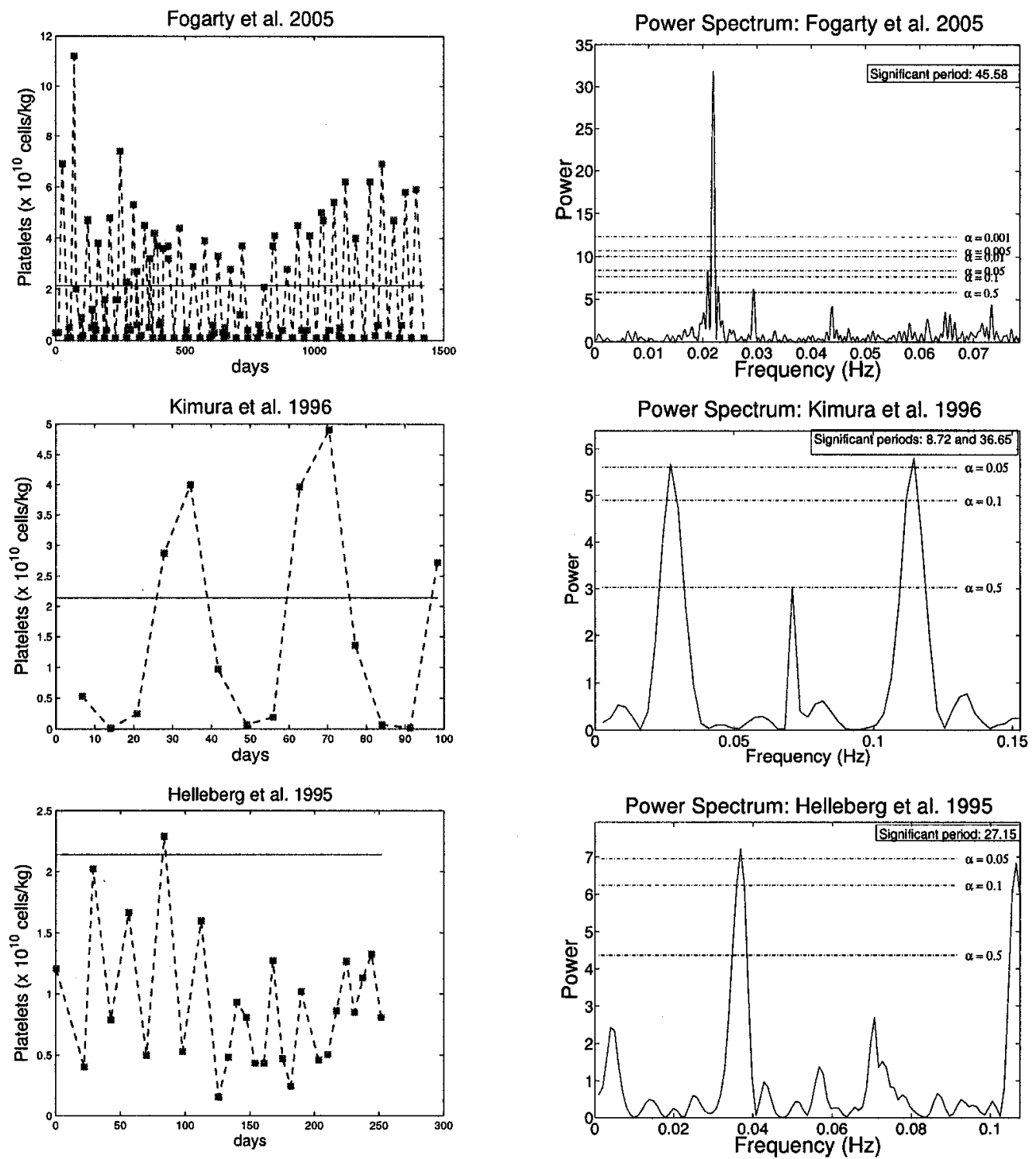

Fig. 2.5 Left-hand panels: Published platelet counts of patients diagnosed with autoimmune CT. The horizontal line shows the normal platelet value in humans $\left(2.14 \times 10^{10}\right.$ cells $\left./ \mathrm{kg}\right)$. Right-hand panels: The corresponding periodograms (power versus frequency). The horizontal lines specify the significance levels. 

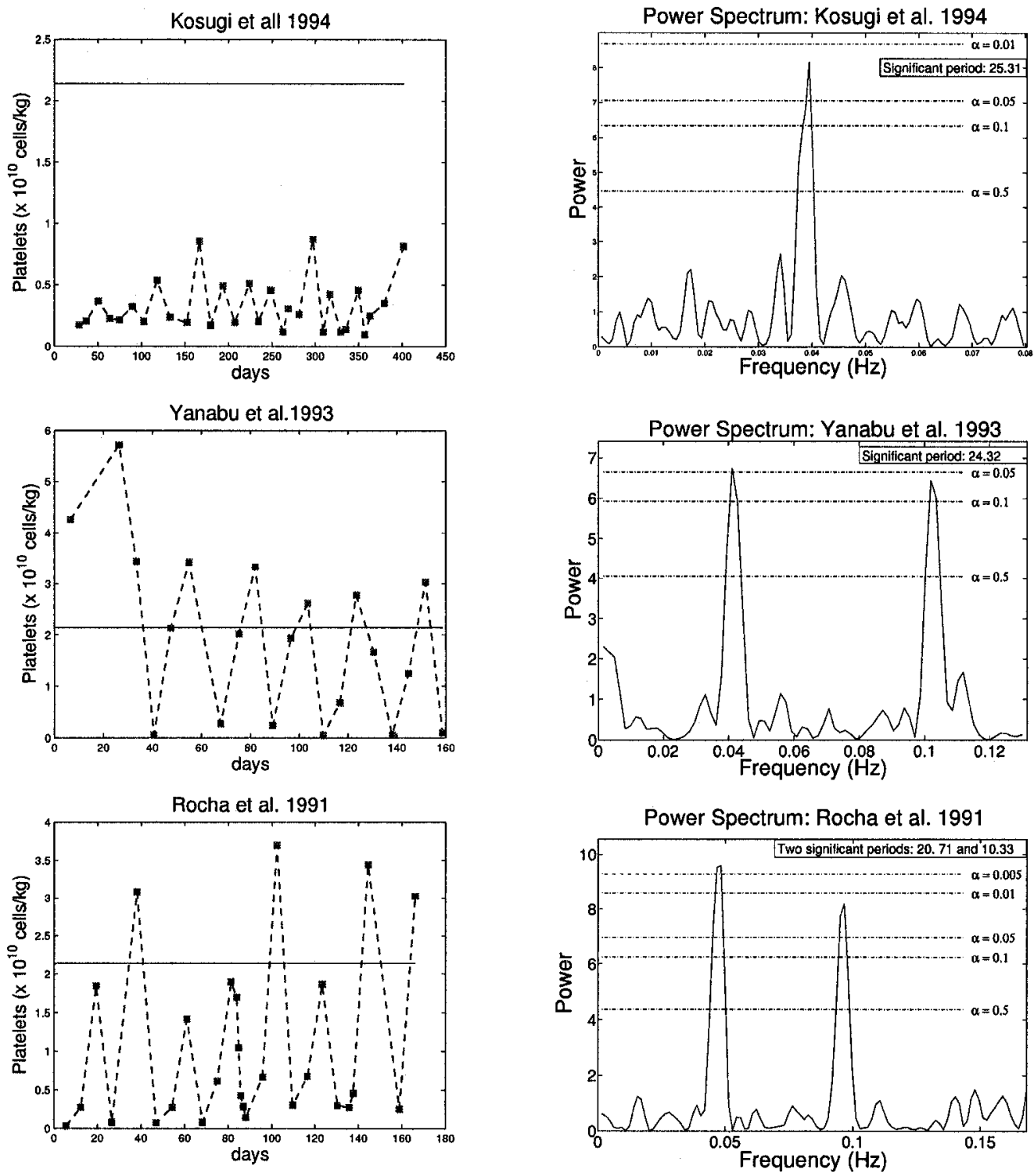

Fig. 2.6 Published platelet counts of patients diagnosed with autoimmune CT. All the other notation as in Figure 2.5. 

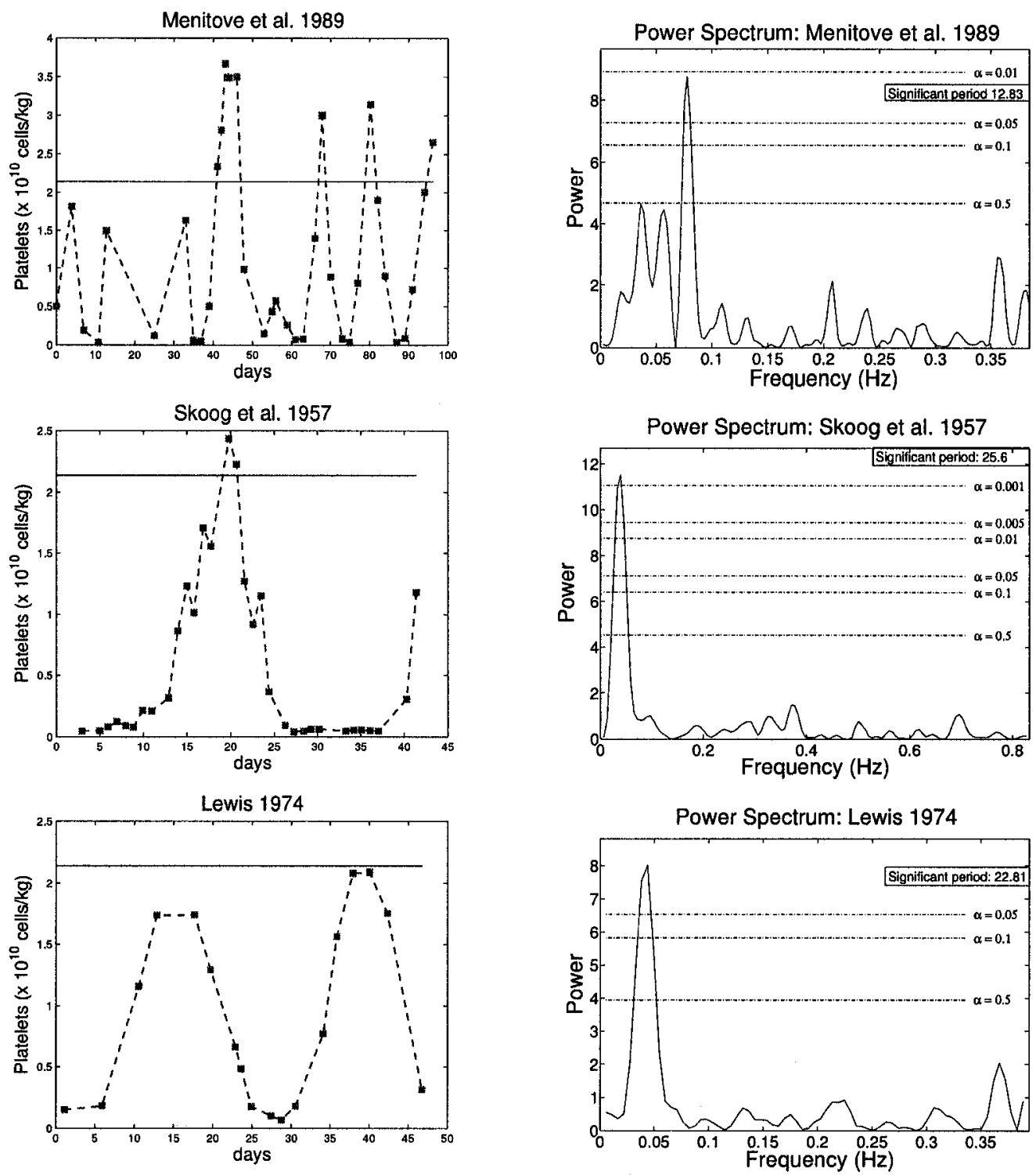

Fig. 2.7 Published platelet counts of patients diagnosed with autoimmune CT (Menitove et al., Skoog et al.) and cyclic TPO levels (Lewis) . All the other notation as in Figure 2.5. 

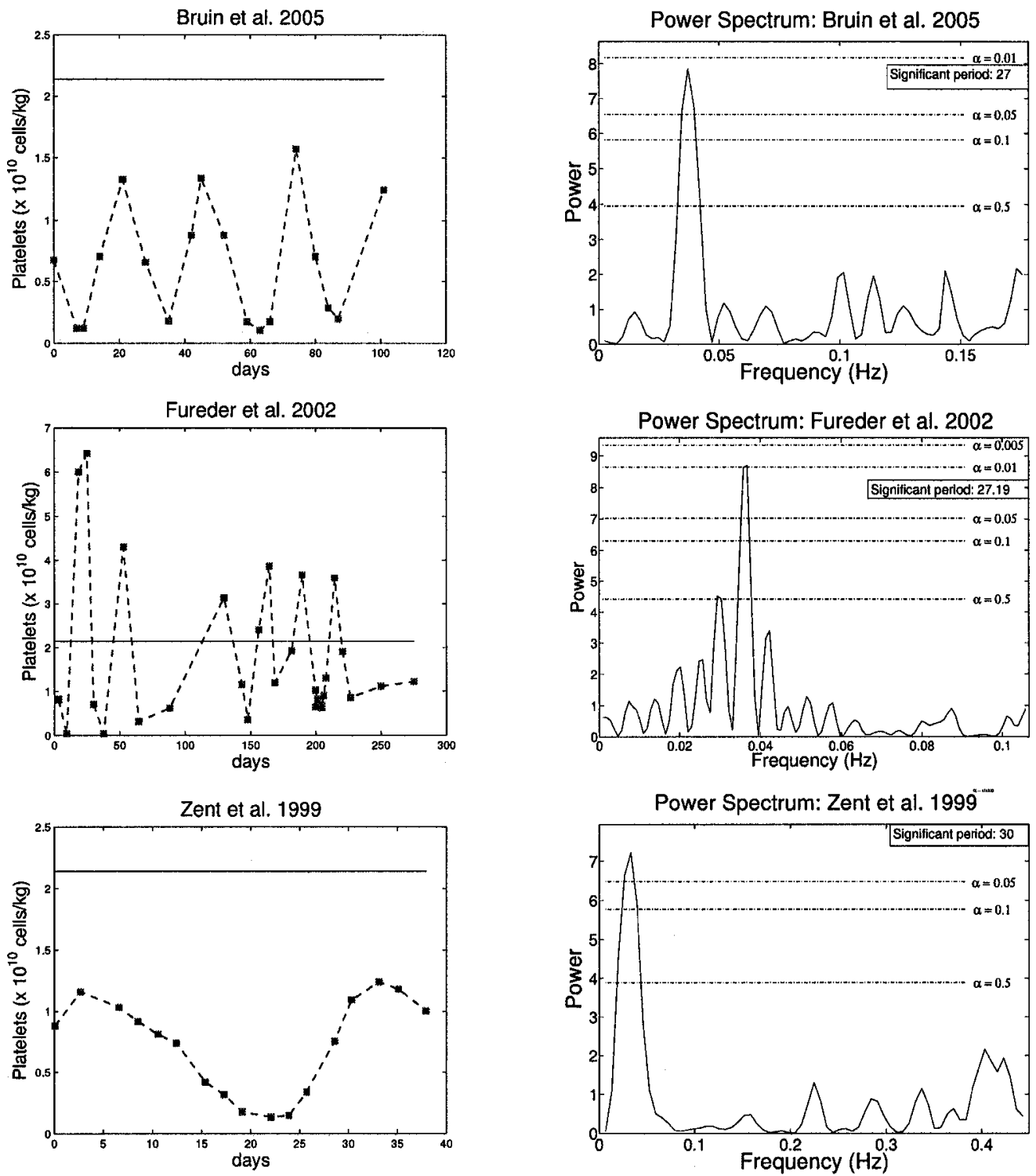

Power Spectrum: Zent et al. $1999^{-0 m}$

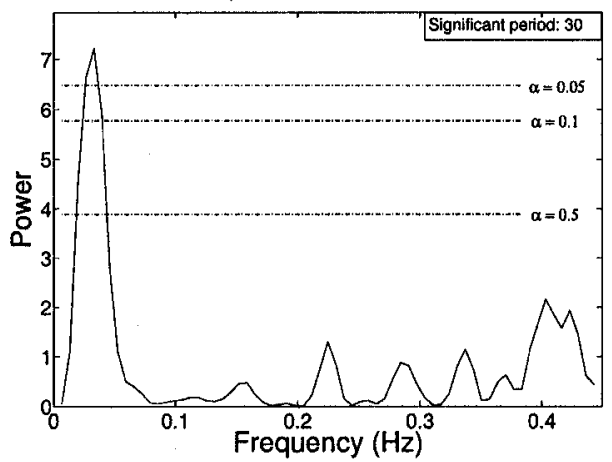

Fig. 2.8 Published platelet counts of patients diagnosed with amegakaryocytic CT. All the other notation as in Figure 2.5. 

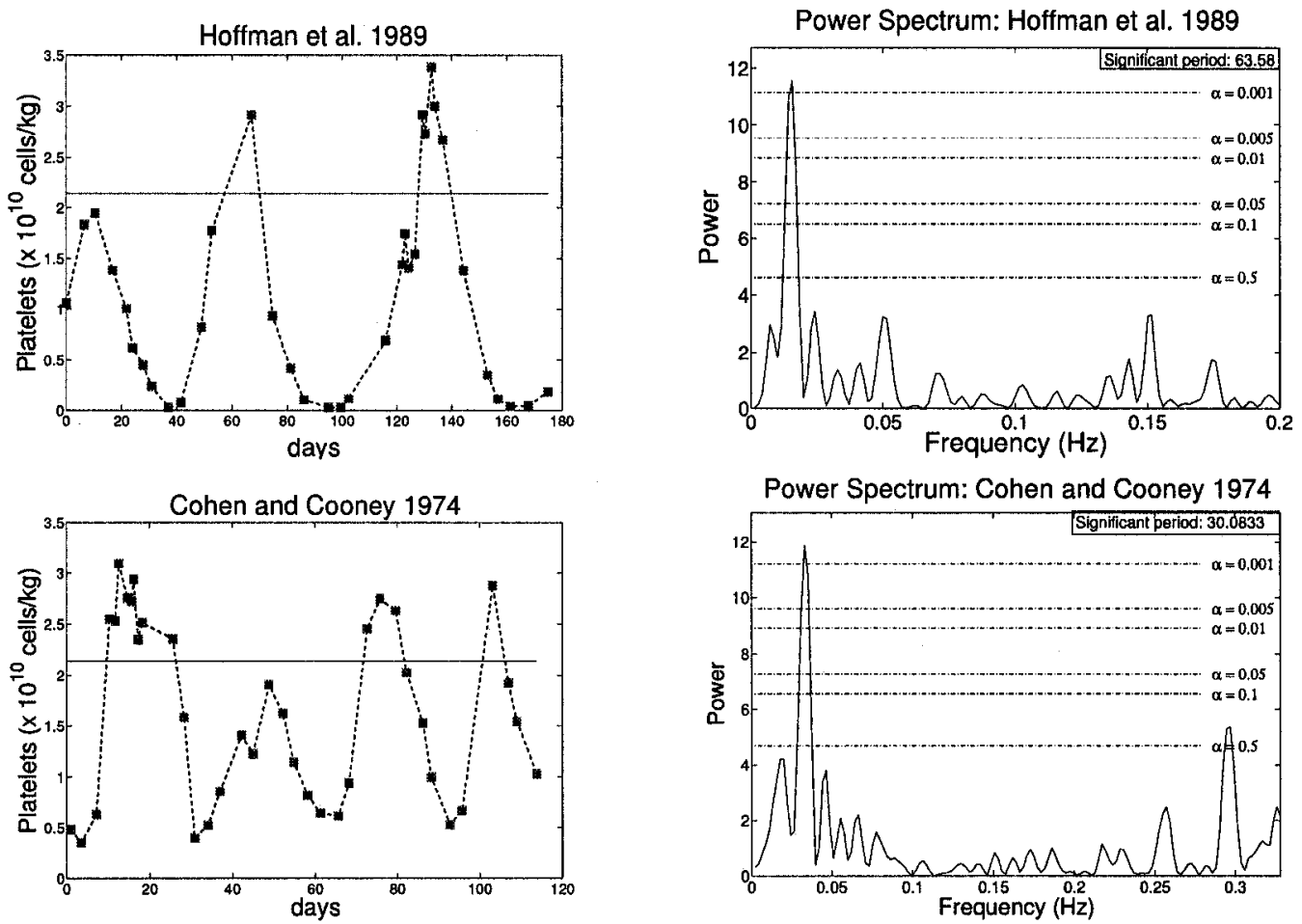

Power Spectrum: Cohen and Cooney 1974
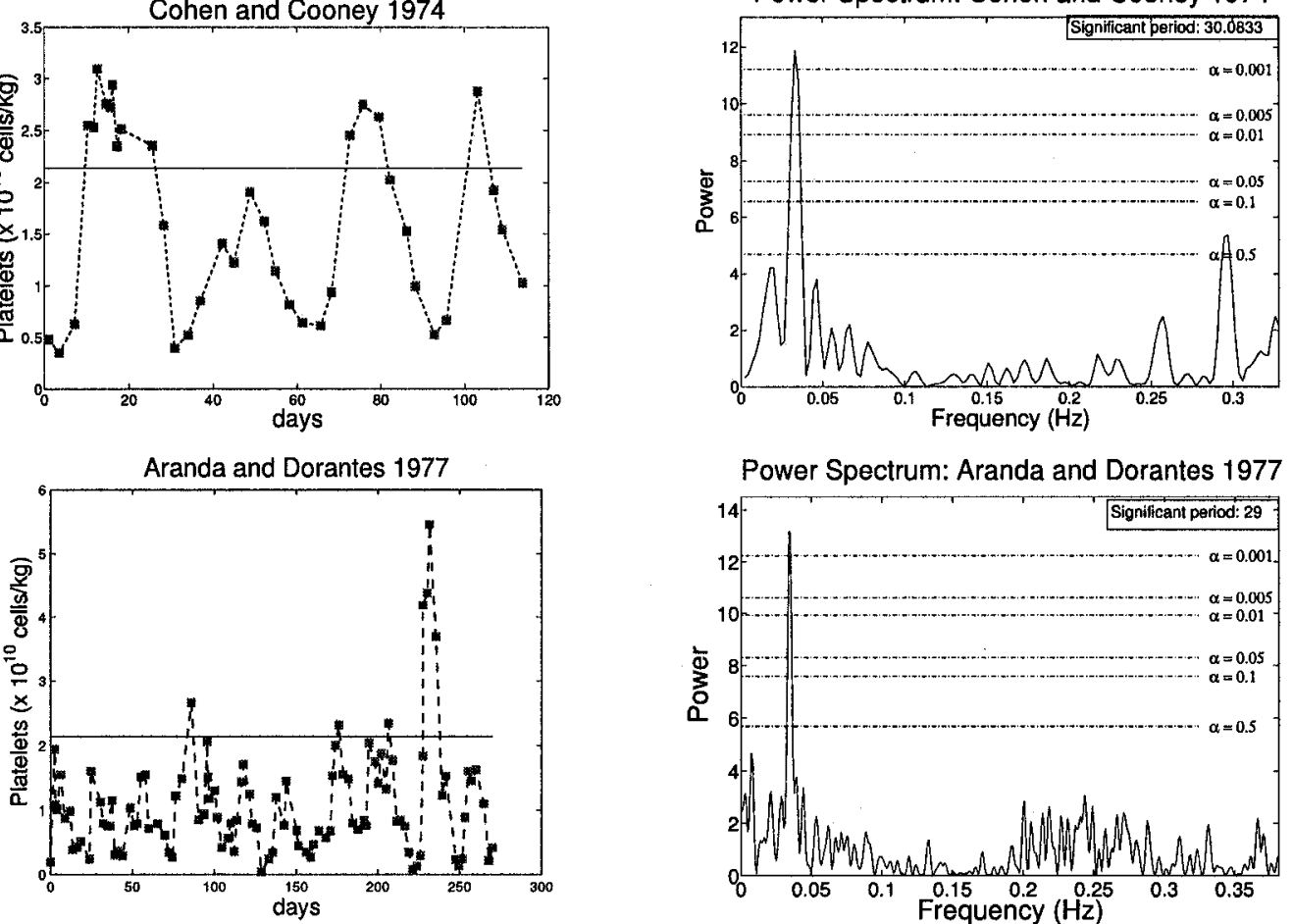

Power Spectrum: Aranda and Dorantes 1977

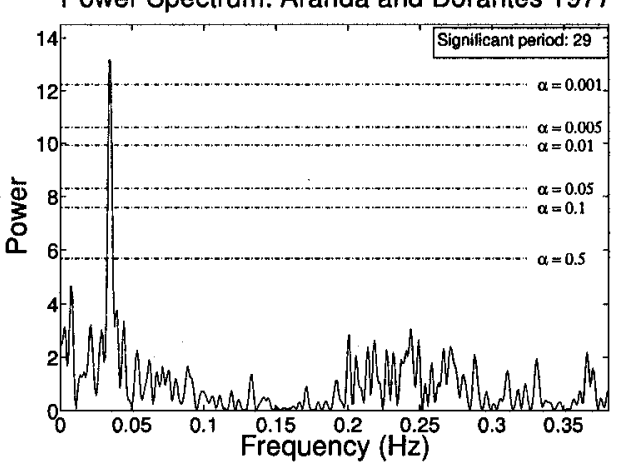

Fig. 2.9 Published platelet counts of patients diagnosed with amegakaryocytic CT. All the other notation as in Figure 2.5. 

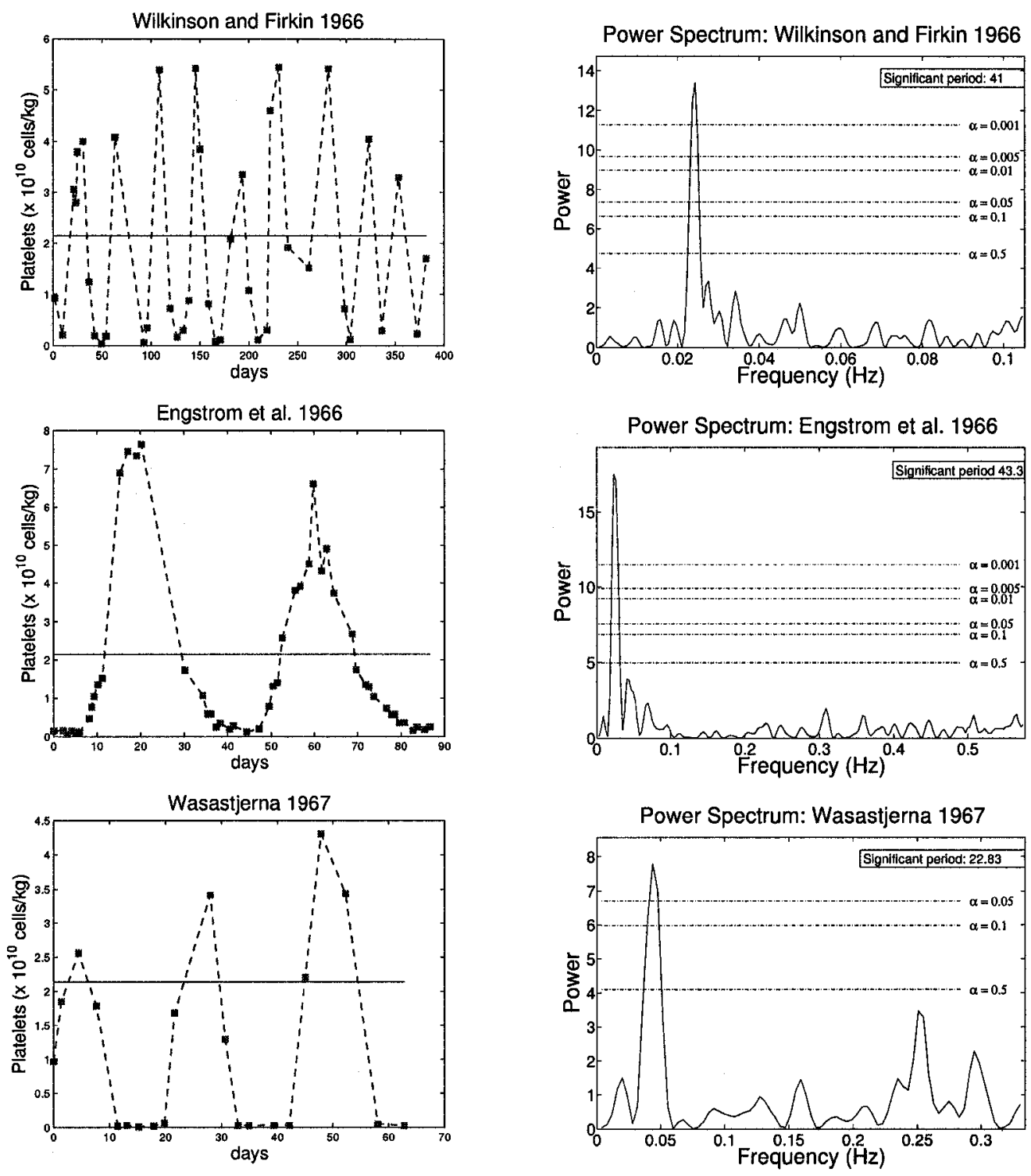

Fig. 2.10 Published platelet counts of patients diagnosed with amegakaryocytic CT. All the other notation as in Figure 2.5. 

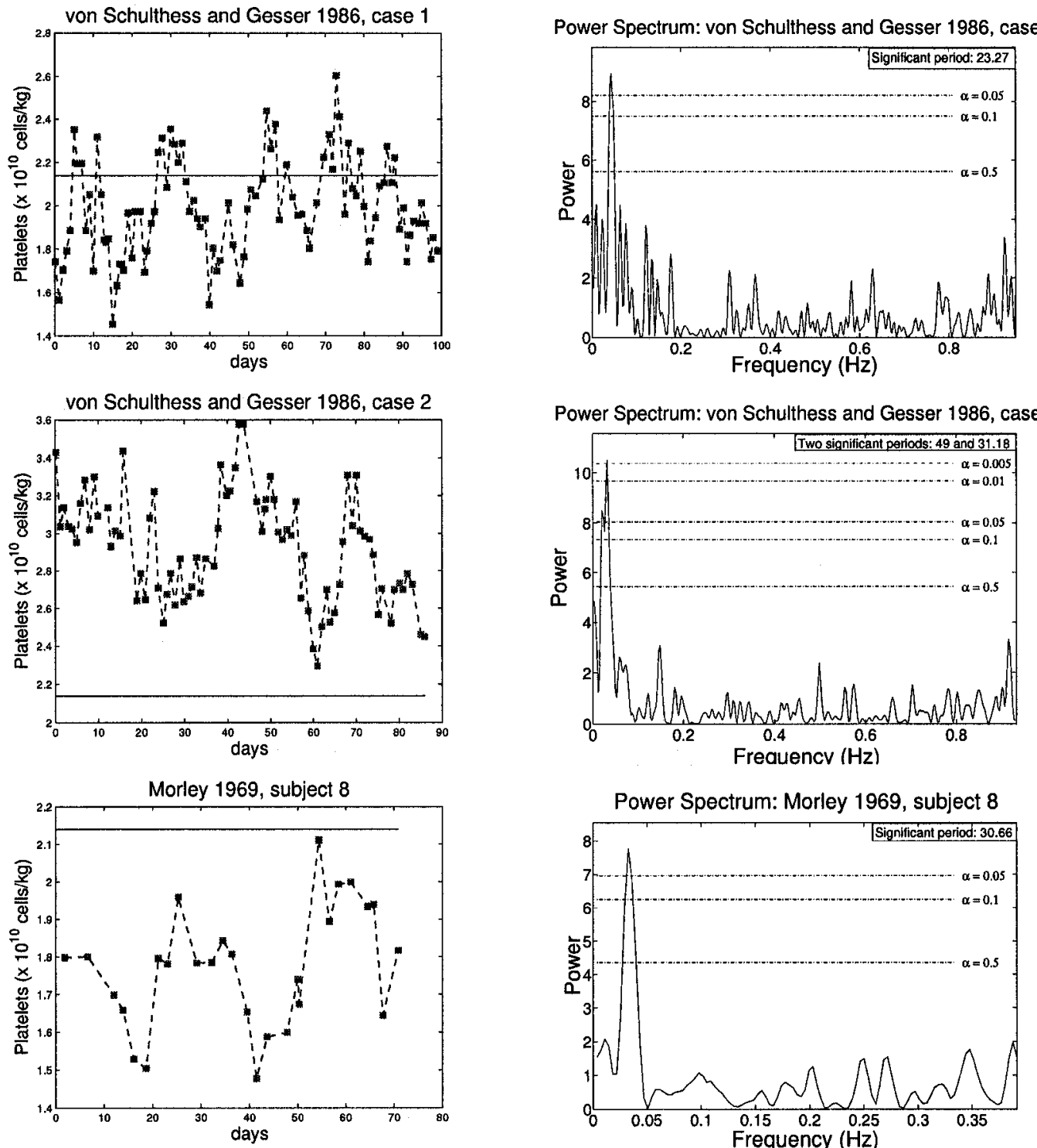

Power Spectrum: Morley 1969, subject 8

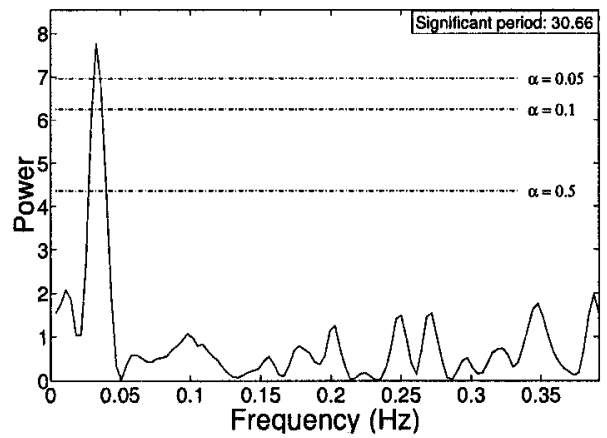

Fig. 2.11 Published platelet counts of asymptomatic patients diagnosed with CT. All the other notation as in Figure 2.5. 


\section{Chapter 3}

\section{A mathematical model of hematopoiesis}

In the last decades mathematical modeling has became a powerful tool in deciphering the mysteries of biological systems. The focus of this chapter is to develop a physiologically realistic model which can help in understanding the onset of oscillations observed in cyclical thrombocytopenia.

\subsection{Differential delay equations}

A variety of mathematical models for biological phenomena are most appropriately framed as differential delay equations (DDE). DDE, also known as differential-difference equations, are a special class of functional delay equations and one of their distinct features is that the evolution rate is described by differential equations which include information on the past history.

DDE were initially introduced in the 18 th century by Laplace and Condorcet and the basic theory concerning the stability of systems described by equations of this type was developed by Pontryagin in 1942. Although now there is a substantial theory available (important works have been written by Bellman and Cooke in 1963, El'sgol'c and Norkin in 1971, Hale in 1977, Kolmanovski and Nosov in 1986, Stépán in 1980), the global knowledge of DDE has not been widely exploited by the scientific community. This is changing with a rapidly growing use of systems with delays in 
applied sciences, mostly in mathematical biology and engineering. The time required for a cell to mature, the time for the nerve impulse to travel along the axon and across the synapse, or the time for the hormonal signals to travel from their site of production to target organs are just few examples of delays in biological systems.

\section{Basic mathematical background}

As usual, $\mathbb{R}^{n}$ denotes the $\mathrm{n}$-dimensional linear vector space over $\mathbb{R}$ with the Euclidian norm $|x|=\sqrt{x_{1}^{2}+\ldots+x_{n}^{2}}$, for $x=\left(x_{1}, \ldots, x_{n}\right) \in \mathbb{R}^{n} . \quad C\left([a, b], \mathbb{R}^{n}\right)$ is the Banach space of continuous functions mapping the interval $[a, b]$ into $\mathbb{R}^{n}$ with the topology of uniform convergence. Let $r$ be a positive real constant. If $[a, b]=[0, r]$ then we will adopt the notation $C=C\left([0, r], \mathbb{R}^{n}\right)$.

For $\sigma \in \mathbb{R}, A \geq 0, x \in C\left([\sigma-r, \sigma+A], \mathbb{R}^{n}\right)$ and $t \in[\sigma, \sigma+A]$ we define $x_{\tau} \in C$ as $x_{\tau}(t):=x(t-\tau)$ for $\tau \in[0, r]$.

Let $\Omega \subset \mathbb{R} \times C$ and $f: \Omega \rightarrow \mathbb{R}^{n}$. An equation of the form

$$
\dot{x}(t)=f\left(t, x_{\tau}(t)\right)
$$

is called a delay differential equation (DDE) on $\Omega$.

If the right hand side of (3.1.1) is independent of $t$ then the system is said to be autonomous. In this case we consider $\sigma=0$. We say that the equation (3.1.1) is linear if $f(t, \phi)=L(t) \phi+h(t)$ with $L(t)$ a linear operator. Equation (3.1.1) is linear homogeneous if $h \equiv 0$ and linear nonhomogeneous if $h \neq 0$.

Definition 1. (Kuang (1993), p.15) A solution of (3.1.1) on $[\sigma-r, \sigma+A]$ is a function $x$ which satisfies simultaneously the following three conditions:

(i) $x \in C\left([\sigma-r, \sigma+A], \mathbb{R}^{n}\right)$,

(ii) $\left(t, x_{\tau}(t)\right) \in \Omega$,

(iii) $x_{\tau}(t)$ satisfies (3.1.1) for $t \in[\sigma, \sigma+A)$.

Definition 2. (Kuang (1993), p.15) For given $\sigma \in \mathbb{R}$ and $\psi \in C$ we say that $x$ is a solution of (3.1.1) with initial value $\psi$ at $\sigma$, or simply a solution through $(\sigma, \psi)$, if there is an $A>0$ such that 
(i) $x$ is a solution of (3.1.1) on $[\sigma-r, \sigma+A]$ in the sense of Definition 1 and

(ii) $x_{\tau}(\sigma)=\psi$.

Theorem 3.1.1. (Kuang (1993), Theorem 2.1, p.19)(Existence) Assume that $\Omega$ is an open subset in $\mathbb{R} \times C$ and $f$ is continuous on $\Omega$. If $(\sigma, \psi) \in \Omega$ then there is a solution of (3.1.1) passing through $(\sigma, \psi)$.

Definition 3. (Kuang (1993), p.19) $f(t, \psi)$ is a Lipschitz function in $\psi$ in a compact set $K$ of $\mathbb{R} \times C$ if there is a constant $k>0$ such that, for any $\left(t, \psi_{i}\right) \in K$, $i \in\{1,2\}$,

$$
\left|f\left(t, \psi_{1}\right)-f\left(t, \psi_{2}\right)\right| \leq\left|\psi_{1}-\psi_{2}\right|
$$

Theorem 3.1.2. (Kuang (1993), Theorem 2.2, p.19)(Uniqueness) Suppose that $\Omega \subset \mathbb{R} \times C$ is open, $f: \Omega \rightarrow \mathbb{R}^{n}$ is continuous, and is $f(t, \psi)$ Lipschitz in $\psi$ in each compact set in $\Omega$. If $(\sigma, \psi) \in \Omega$, then there is a unique solution of (3.1.1) through $(\sigma, \psi)$.

For a careful derivation of the these results the reader is referred to (57).

Remark 3. (3.1.1) is a very general type of equation and includes

(i) ordinary differential equations $(r=0)$

$$
\dot{x}(t)=F(x(t))
$$

(ii) differential difference equations

$$
\dot{x}(t)=f\left(t, x(t), x\left(t-\tau_{1}\right), \ldots, x\left(t-\tau_{m}\right)\right)
$$

Due to the dependency on the past history, a solution is not uniquely defined by the value of $x(t)$ at some specific moment $t$. One has to specify an initial solution over an interval of length $\tau$, where $\tau=\max _{i=\overline{1, m}} \tau_{i}$. 


\subsection{Model development}

To our knowledge, the first mathematical model of hematopoietic regulation had been proposed by Lajtha et al. (63) in 1962 to describe stem cell proliferation and differentiation. This paper stimulated many biologists and biomathematicians, and since then numerous concepts have been cast into the frame of the mathematical models.

Recently, Colijn and Mackey (18) synthesized a $G_{0}$-type model for the HSC dynamics (66) with the mathematical models for leukocytes ((8), (43), (45)), erythrocytes $((6),(67),(69))$, and platelets $((5),(83))$ into a comprehensive model of hematopoiesis. Their work was motivated by the existence of the dynamical diseases which provide the opportunity to understand the regulatory mechanism in early hematopoiesis and its effect on the blood cell lines. The model presented in (18) not only captures the essential characteristics of hematopoiesis but also offers an advantage comparable with the earlier models consisting of one cell line or one line coupled to the stem cells. In the framework offered by Colijn and Mackey it is possible to analyze the effect of stem cell destabilization on the whole system and the echoes of one blood cell line fluctuations on the other compartments. For example, hematological diseases like CN (19) and PCML (18) involve oscillations in all circulating hematological cells and their cause is better understood through a four-compartment model.

\section{Colijn \& Mackey model (18) versus our model}

Since the model of Colijn \& Mackey (18) has been shown to display the features of two dynamical diseases (PCML in (18) and CN in (19)), we chose it as a starting point in our quantitative investigation of CT. Extensive numerical experiments showed that any induced oscillations in the platelet compartment destabilize the neutrophil line. Our successive attempts lead to the conclusion that the hematopoietic model in this form cannot generate oscillatory solutions in platelet compartment while maintaining all the other variables at their steady state levels. The main cause of this phenomenon is the assumed dependence of the platelet differentiation rate on the number of circulating platelets. Since the molecular mechanisms of platelet regula- 


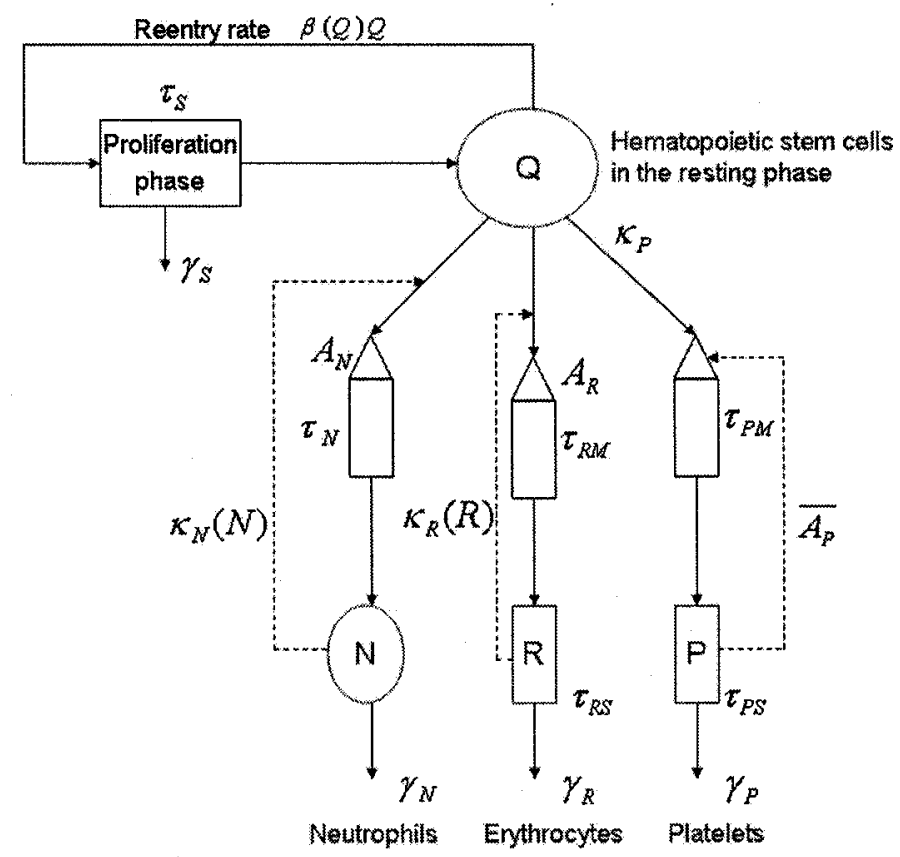

Fig. 3.1 The mathematic model of hematopoiesis. Solid arrows show the movement of cells, while dashed lines reflect the feedback functions.

tion are not completely elucidated, given the similarities between erythropoiesis and thrombopoiesis, the authors (18) assumed that the platelet differentiation rate follows the same mechanism as in the case of erythrocytes, and depends on the number of circulating cells. Searching the literature, we found that experimental data suggests that the megakaryocyte compartment is maintained by an approximately constant influx of progenitor cells (13). Therefore, in our modeling work, we assume that the platelet differentiation rate is constant. For this reason, we will derive a new equation for the platelet dynamics and while maintaining the structure of the stem cell, neutrophil and erythrocyte compartments given in (18) (see Figure 3.1 for a cartoon representation). In the following, we present a brief model development, with a particular emphasis on the platelet compartment. The pluripotential, non-proliferating stem cells, the circulating neutrophils, platelets and erythrocytes are denoted by $Q$, $\mathrm{N}, \mathrm{P}$ and $\mathrm{R}$, respectively. We adopt the notation convention $X_{\tau}(t):=X(t-\tau)$ for any variable $X$. 


\section{Stem cell compartment}

The stem cells in the resting phase (or $G_{0}$ phase) of the cell cycle do not divide. There are only two ways that they can exit the non-proliferating compartment: either enter the proliferating phase at a rate $\beta(Q)$ or differentiate into erythrocytes, neutrophils and platelets at rate $\kappa_{R}, \kappa_{N}$ and $\kappa_{P}$, respectively. After re-entering the proliferating phase, the cells divide, taking a time $\tau_{S}$ to do so. Immediately after, the two daughter cells move into the resting phase. Using this notation, we can write a balance equation stating that the rate of change of HSC number is the difference between their production and their loss:

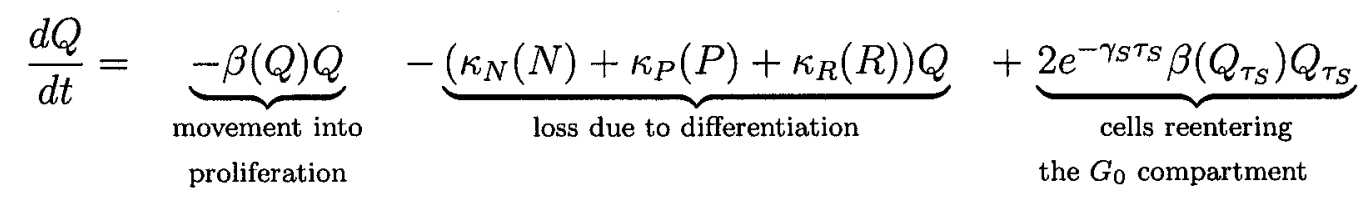

The last term contains the gain due to the movement of cells into the $G_{0}$ phase one generation time ago. The factor 2 describes the division of each cell into two daughter cells and $e^{-\gamma_{S} \tau_{S}}$ corrects for the probability of the loss from the proliferating population.

\section{Platelet compartment}

Once a pluripotential stem cell is committed to this pathway, it undergoes a series of nuclear divisions and enters a maturation phase for $\tau_{P M}$ days before being released into circulation. After $\tau_{P S}$ days the platelets are primarily lost to senescence at a rate of $\gamma_{P}$ per day. Colijn \& Mackey (18) proposed the following DDE to model the platelet variation:

$$
\frac{d P}{d t}=-\gamma_{P} P+A_{P}\left\{\kappa_{P}\left(P_{\tau_{P M}}\right) Q_{\tau_{P M}}-e^{-\gamma_{P} \tau_{P S}} \kappa_{P}\left(P_{\tau_{P M}+\tau_{P S}}\right) Q_{\tau_{P M}+\tau_{P S}}\right\},
$$

where the platelet differentiation rate $\kappa_{P}$ is given by

$$
\kappa_{P}(P)=\frac{\overline{\kappa_{r}}}{1+K_{P} P^{r}} .
$$


We use a similar reasoning but under the approximation that the platelet differentiation rate is constant. Therefore the change in the platelet counts is given by:

$$
\frac{d P}{d t}=-\gamma_{P} P+\bar{A}_{P} \kappa_{P}\left(Q_{\tau_{P M}}-e^{-\gamma_{P} \tau_{P S}} Q_{\tau_{P M}+\tau_{P S}}\right) .
$$

The main agent controlling the the peripheral platelet regulatory system through $\overline{A_{P}}$ (the average number of platelets released per megakaryocyte) is thrombopoietin. Let $V(t)$ denote the megakaryocyte volume and $T(t)$ the TPO concentration at the time $t$. The available experimental data allows us to suppose that

(i) $\overline{A_{P}}(t) \propto V(t)$ and

(ii) $V(t)$ obeys the ordinary differential equation $\frac{d V}{d t}(t)=\mu T(t) V(t)$.

Solving the above equation we get:

$$
V(t)=V\left(t-\tau_{P M}\right) e^{\mu \int_{t-\tau_{P M}}^{t} T\left(t^{\prime}\right) d t^{\prime}}
$$

Since the differentiation rate $\kappa_{P}$ is constant we can consider $V\left(t-\tau_{P M}\right)=V_{0}$. Hence

$$
\overline{A_{P}}(t)=A_{0} e^{\mu \int_{t-\tau_{P M}}^{t} T\left(t^{\prime}\right) d t^{\prime}},
$$

where $A_{0}$ denotes the minimal number of platelets produced per megakaryocyte. But

$$
\bar{T}(t):=\frac{1}{\tau_{P M}} \int_{t-\tau_{P M}}^{t} T\left(t^{\prime}\right) d t^{\prime}
$$

represents the average TPO concentration at the moment $t$. Hence

$$
\overline{A_{P}}(t)=A_{0} e^{\mu \tau_{P M} \bar{T}(t)}
$$

Santillán et al. (83) model the TPO concentration under the assumption that the number of megakaryocytes of age zero entering from the stem cell compartment is directly proportional to the thrombopoietin levels. They find:

$$
\frac{d T}{d t}=\frac{a}{1+K_{P} P^{r}}-\kappa T
$$


Additionally, if we consider that TPO concentration is in dynamic equilibrium with the number of circulating platelets then $\frac{d T}{d t}=0$. Therefore

$$
T \propto \frac{1}{1+K_{P} P^{r}} \quad \text { which implies } \quad T=\frac{T_{\max }}{1+K_{P} P^{r}} .
$$

$T_{\text {max }}$ represents the maximum TPO level in blood.

\section{Erythrocyte compartment}

The erythrocyte and platelet dynamics share common features and display some important differences. The primary difference between erythropoiesis and thrombopoiesis is related to the development of the precursor cells. In erythropoiesis, the stem cells undergo rapid proliferation and differentiation until they become reticulocytes, which mature and become circulating erythrocytes. In thrombopoiesis, the stem cells proliferate until they reach the stage of megakaryocytes, which no longer proliferate but undergo endoreduplication (83). Therefore the total variation of erythrocytes is modeled by:

$$
\frac{d R}{d t}=\underbrace{-\gamma_{R} R}_{\text {random loss }}+\underbrace{A_{R} \kappa_{R}\left(R_{\tau_{R M}}\right) Q_{\tau_{M}}}_{\begin{array}{c}
\text { cells entering from the } \\
\text { stem cell compartment }
\end{array}}-\underbrace{A_{R} e^{-\gamma_{R} \tau_{R S}} \kappa_{R}\left(R_{\tau_{R M}+\tau_{R S}}\right) Q_{\tau_{R M}+\tau_{R S}}}_{\text {loss due to senescence }}
$$

All the notation (except for $A_{R}$ ) is analogous to those in equation (3.2.1). $A_{R}$ is the dimensionless parameter corresponding to the amplification stage due to cell division.

\section{Neutrophil compartment}

As the neutrophil precursors differentiate, their number is amplified by a constant factor $A_{N}$ which accounts for the stages of cell division. After $\tau_{N}$ days they become mature and are released into circulation. The neutrophils are randomly lost at the rate $\gamma_{N}$. Their dynamics is governed by the equation: 


$$
\frac{d N}{d t}=\underbrace{-\gamma_{N} N}_{\text {random loss }}+\underbrace{A_{N} \kappa_{N}\left(N_{\tau_{N}}\right) Q_{\tau_{N}}}_{\begin{array}{c}
\text { cells entering from } \\
\text { the stem cell compartment }
\end{array}}
$$

\section{Forms of the feedback functions and of the stem cells re-entry rate into proliferation}

Hematopoiesis is a homeostatic system and depends on carefully balanced negative feedback loops. The last step of our model construction is to define appropriate forms for the feedback functions and the stem cell re-entry rate into proliferation. Since it takes several days for cells to proliferate or to mature these feedback effects are delayed.

In their age-structured erythropoietic model, Mahaffy et al. (69) assume that the numbers of precursors entering the erythrocytes line is linearly proportional to the erythropoietin concentration $(\mathrm{E})$ and derive the equation:

$$
\frac{d E}{d t}=\frac{a}{1+K R_{r}^{m}}-\kappa E
$$

We preserve this approximation and assume that the erythropoietin levels are in dynamic equilibrium with the erythrocytes numbers. Therefore

$$
\frac{d E}{d t}=0 \quad \text { and } \quad \kappa_{R}(R) \propto E \propto \frac{1}{1+K R_{r}^{m}} .
$$

which implies $\kappa_{R}(R)=\frac{\bar{\kappa}_{r}}{1+K R_{r}^{m}}$.

The leukocyte feedback function $\kappa_{N}$ is derived by Bernard et al. (8) based on enzyme kinetic principles as:

$$
\kappa_{N}(N)=f_{0} \frac{\theta_{1}^{n}}{\theta_{1}^{n}+N^{n}}
$$

The mitotic reentry rate from $G_{0}$ phase into proliferation should smoothly decrease when $\mathrm{Q}$ increases, have a finite maximum, adjustable inflection point and slope. A convenient function that captures this behavior and has helpful analytic properties is 
the Hill function. Mackey et al. (67) write the stem cells re-entry rate to proliferative phase as:

$$
\beta(Q)=k_{0} \frac{\theta_{2}^{s}}{\theta_{2}^{s}+Q^{s}} .
$$

$k_{0}$ is the maximum rate of cell movement from the resting phase into proliferation, $\theta_{2}$ represents the $G_{0}$ stem cell population at which the rate of the cell movement from the resting phase into proliferation is half of its maximal value $k_{0}$, and $s$ is the sensitivity rate of reintroduction. Obviously, $\beta^{\prime}(t)<0$ for $t>0$ and $\lim _{t \rightarrow \infty} \beta(t)=0$.

Summarizing, the equations comprising the model are:

$$
\left\{\begin{array}{l}
\frac{d Q}{d t}=-\left(\beta(Q)+\kappa_{N}(N)+\kappa_{P}+\kappa_{R}(R)\right) Q+2 e^{-\gamma_{S} \tau_{S}} \beta\left(Q_{\tau_{S}}\right) Q_{\tau_{S}} \\
\frac{d N}{d t}=-\gamma_{N} N+A_{N} \kappa_{N}\left(N_{\tau_{N}}\right) Q_{\tau_{N}} \\
\frac{d R}{d t}=-\gamma_{R} R+A_{R}\left(\kappa_{R}\left(R_{\tau_{R M}}\right) Q_{\tau_{R M}}-e^{\left.-\gamma_{R} \tau_{R S} \kappa_{R}\left(R_{\tau_{R M}+\tau_{R S}}\right) Q_{\tau_{R M}+\tau_{R S}}\right)}\right. \\
\frac{d P}{d t}=-\gamma_{P} P+\bar{A}_{P} \kappa_{P}\left(Q_{\tau_{P M}}-e^{-\gamma_{P} \tau_{P S}} Q_{\tau_{P M}+\tau_{P S}}\right)
\end{array}\right.
$$

where

$$
\begin{aligned}
\beta(Q) & =k_{0} \frac{\theta_{2}{ }^{s}}{\theta_{2}{ }^{s}+Q^{s}} \\
\kappa_{N}(N) & =f_{0} \frac{\theta_{1}{ }^{n}}{\theta_{1}{ }^{n}+N^{n}} \\
\kappa_{R}(R) & =\frac{\kappa_{r}}{1+K_{r} R^{m}} \\
T & =\frac{T_{\max }}{1+K_{P} P^{r}} \\
\bar{T}(t) & =\frac{1}{\tau_{P M}} \int_{t-\tau_{P M}}^{t} T\left(t^{\prime}\right) d t^{\prime} \\
\overline{A_{P}}(t) & =A_{0} e^{\mu \tau_{P M} \bar{T}(t)}
\end{aligned}
$$




\subsection{Parameter estimation}

The parameter estimation is one of the most important aspects of our modeling work since the biologically relevant choice of the parameters is crucial to establish the onset of oscillations observed in CT. Using experimental data published in the literature we can evaluate all the parameters outside the negative feedback functions.

Bernard et al. (8) derived all the values corresponding to the stem cell and leukocyte compartments. We use their evaluations except for the dimensionless amplification parameter $A_{N}$ and the Hill coefficient $f_{0} . A_{N}$ has been estimated by Mackey (68) as 300,000 and we use this value. The normal range for $f_{0}$ is $0.4-1.5$ (43). In (8) the authors choose $f_{0}=0.8$ only to make their model fit the data. Since our model estimates

$$
f_{0}=\kappa\left(N_{*}\right) \frac{\theta_{1}+N_{*}}{\theta_{1}}=\frac{\gamma_{N} N_{*}}{A_{N} Q_{*}} \times \frac{\theta_{1}+N_{*}}{\theta_{1}}=0.4
$$

we set $f_{0}=0.4$.

The erythrocyte parameters are evaluated by Mahaffy et al. (69) based on experimental human data. To their information we add the dimensionless amplification parameter $A_{R}=563,000((12),(18))$ and the Hill coefficient $\bar{\kappa}_{r}$ mathematically estimated in (18) as 1.1 days $^{-1}$.

Santillán et al. (83) give the normal values of $\gamma_{P}, \tau_{P M}, \tau_{P S}$ and the mean platelet count $P_{*}=2.5 \times 10^{8}$ cells $/ \mathrm{ml}$. Using the fact that $70 \mathrm{~kg}$ adult has $6 \mathrm{~L}$ of blood we find $P_{*}=2.14 \times 10^{10}$ cells $/ \mathrm{kg}$. For the platelet control dynamics we need to estimate few more parameters: the effective growth rate of megakaryocytes $(\mu)$, the minimum number of platelets produced per megakaryocyte $\left(A_{0}\right)$, the differentiation rate $\left(\kappa_{P}\right)$ and the maximum TPO concentration $\left(T_{m a x}\right)$. The experimental measurements show that one mature megakaryocyte can give rise to $1,000-5,000$ platelets (12). Therefore we choose $A_{0}=1000$ platelets/megakaryocyte. By fitting the TPO concentrations versus the platelet count, Santillán et al. (83) evaluate

$$
T_{*}=0.005 \mathrm{U} / \mathrm{ml}, \quad T_{\max }=32.18 T_{*}, \quad K_{P}=\frac{31.18}{\left(P_{*}\right)^{r}}, \quad \text { and } \quad r=1.29 \text {. }
$$


Converting to cells $/ \mathrm{kg}$ we get

$$
T_{*}=0.428 \mathrm{U} / \mathrm{kg}, \quad T_{m a x}=13.773 \mathrm{U} / \mathrm{kg} \quad \text { and } \quad K_{P}=11.66\left(\times 10^{10} \mathrm{cells} / \mathrm{kg}\right)^{-r} .
$$

Deriving the model parameters we have to make sure that there is a balance between the influx and the efflux from the resting phase of the stem cell compartment. Mathematically, this means that at steady state the following relation should be satisfied:

$$
-\left(\kappa_{R}^{*}+\kappa_{N}^{*}+\kappa_{P}\right)+2 e^{-\gamma_{S} \tau_{S}} \beta_{*}=0 .
$$

The above equation determines the normal value of the platelet differentiating rate:

$$
\kappa_{P}=\left(2 e^{-\gamma_{S} \tau_{S}}-1\right) \beta_{*}-\kappa_{R}^{*}-\kappa_{N}^{*}=0.028 \text { days }^{-1} .
$$

Once all the other parameters are estimated, it is easy to derive the effective growth rate of megakaryocytes:

$$
\mu=\frac{1}{T_{*} \tau_{P M}} \ln \frac{{\overline{A_{P}}}^{*}}{A_{0}}=\frac{1}{T_{*} \tau_{P M}} \ln \frac{\gamma_{P} P_{*}}{\kappa_{P} A_{0} Q_{*}\left(1-e^{\left.-\gamma_{P} \tau_{P S}\right)}\right.}=1.7836 \quad(\mathrm{U} \text { days } / \mathrm{kg})^{-1}
$$




\begin{tabular}{|c|c|c|c|c|}
\hline Parameter Name & Value Used & Unit & Source & Physiological signification \\
\hline \multicolumn{5}{|l|}{$\begin{array}{c}\text { Stem Cell } \\
\text { Compartment }\end{array}$} \\
\hline$Q_{*}$ & 1.1 & $10^{6}$ cells $/ \mathrm{kg}$ & $(8)$ & \multirow{6}{*}{$\begin{array}{c}\text { normal stem cell count } \\
\text { apoptosis rate } \\
\text { stem cell proliferation time } \\
\text { maximum rate of cell movement } \\
\text { from the } G_{0} \text { into proliferation } \\
G_{0} \text {-stem cell population at which the rate of cell } \\
\text { movement from } G_{0} \text { into proliferation is } \frac{k_{0}}{2} \\
\text { controls the sensitivity of the mitotic } \\
\text { reentry rate } \beta \text { to changes in the size of } G_{0}\end{array}$} \\
\hline$\gamma_{S}$ & 0.07 & days $^{-1}$ & (8) & \\
\hline$\tau_{S}$ & 2.8 & days & (8) & \\
\hline$k_{0}$ & 8.0 & days $^{-1}$ & (8) & \\
\hline$\theta_{2}$ & 0.095 & $10^{6}$ cells $/ \mathrm{kg}$ & (8) & \\
\hline NT & 2 & (none) & (8) & \\
\hline \multicolumn{5}{|l|}{$\begin{array}{c}\text { Neutrophil } \\
\text { Compartment }\end{array}$} \\
\hline$N_{*}$ & 6.9 & $10^{8}$ cells $/ \mathrm{kg}$ & $(8),(18)$ & \multirow{7}{*}{$\begin{array}{c}\text { normal neutrophil count } \\
\text { random loss of circulating neutrophils } \\
\text { leukocytes maturation time } \\
\text { dimensionless amplification parameter }\end{array}$} \\
\hline$\gamma_{N}$ & 2.4 & days $^{-1}$ & $(8),(18)$ & \\
\hline$\tau_{N}$ & 3.5 & days & $(8),(18)$ & \\
\hline$A_{N}$ & $3000 \times 10^{2}$ & (none) & $(68)$ & \\
\hline$f_{0}$ & 0.4 & days $^{-1}$ & (18) & \\
\hline$\theta_{1}$ & 0.36 & $10^{8}$ cells $/ \mathrm{kg}$ & $(8),(18)$ & \\
\hline$n$ & 1 & (none) & $(8),(18)$ & \\
\hline \multicolumn{5}{|l|}{$\begin{array}{l}\text { Erythrocyte } \\
\text { Compartment }\end{array}$} \\
\hline$R_{*}$ & 3.5 & $10^{11}$ cells $/ \mathrm{kg}$ & $(69),(18)$ & \multirow{8}{*}{$\begin{array}{l}\text { normal erythrocyte count } \\
\text { random loss of circulating erythrocytes } \\
\text { erythrocytes maturation time } \\
\text { erythrocytes aging time to senescence } \\
\text { dimensionless amplification parameter }\end{array}$} \\
\hline$\gamma_{R}$ & 0.001 & days $^{-1}$ & $(69),(18)$ & \\
\hline$\tau_{R M}$ & 6 & days & $(69),(18)$ & \\
\hline$\tau_{R S}$ & 120 & days & $(69),(18)$ & \\
\hline$A_{R}$ & $5.63 \times 10^{5}$ & (none) & $(12),(18)$ & \\
\hline $\bar{\kappa}_{r}$ & 0.5 & days $^{-1}$ & (18) & \\
\hline$K_{r}$ & 0.0382 & $\left(10^{11} \text { cells } / \mathrm{kg}\right)^{-m}$ & $(69),(18)$ & \\
\hline$m$ & 6.96 & (none) & $(69),(18)$ & \\
\hline \multicolumn{5}{|l|}{$\begin{array}{c}\text { Platelet } \\
\text { Compartment }\end{array}$} \\
\hline$P_{*}$ & 2.14 & $10^{10}$ cells $/ \mathrm{kg}$ & $(83),(18)$ & normal platelet count \\
\hline$\gamma_{P}$ & 0.15 & days $^{-1}$ & $(83),(18)$ & random loss of circulating platelets \\
\hline$\tau_{P M}$ & 7 & days & $(83),(18)$ & platelet maturation time \\
\hline$\tau_{P S}$ & 9.5 & days & $(83),(18)$ & platelet aging time to senescence \\
\hline$\mu$ & 1.7836 & $(\mathrm{U} \text { days } / \mathrm{kg})^{-1}$ & calculated & effective growth rate of megakaryocytes \\
\hline$A_{0}$ & $0.1 \times 10^{4}$ & (none) & (12) & $\begin{array}{l}\text { minimum number of platelets produced } \\
\text { per megakaryocyte when TPO is } \simeq 0\end{array}$ \\
\hline$\kappa_{P}$ & 0.028 & days $^{-1}$ & calculated & platelet differentiating rate \\
\hline$K_{P}$ & 11.68 & $\left(10^{10} \text { cells } / \mathrm{kg}\right)^{-r}$ & $(83),(18)$ & \\
\hline$r$ & 1.29 & (none) & $(83),(18)$ & \\
\hline$T_{*}$ & 0.428 & $\mathrm{U} / \mathrm{kg}$ & $(83)$ & normal TPO concentration \\
\hline$T_{\max }$ & 13.773 & $\mathrm{U} / \mathrm{kg}$ & calculated & maximum possible TPO concentration \\
\hline
\end{tabular}

Table 3.1 Estimated equilibrium values for normal subjects. 


\section{Chapter 4}

\section{Mathematical analysis of the model}

In the previous chapter we developed a mathematical model of hematopoiesis consistent with the biological framework and evaluated the parameters based on the clinical findings published in the literature. For correct predictions, the DDE system (3.2.5) should also satisfy the mathematical requirements. This chapter is devoted to the analytical study of the mathematical model. The first natural steps are the existence and uniqueness of solutions, followed by the investigation of steady states. Since linear stability analysis reveals the dynamical behavior of the system in the neighborhood of a stationary solution, we extract the all the possible information from the characteristic equation.

\subsection{Model properties}

Let $\tau:=\max \left\{\tau_{S}, \tau_{N}, \tau_{R M}, \tau_{R S}, \tau_{P S}, \tau_{P M}\right\}$. Given the dependence on the past history, the equations (3.2.5) should be defined for $t \geq \tau$ and the initial conditions should be given on the interval $[0, \tau]$. For our work, the most two important model properties are illustrated in the following propositions:

Proposition 4.1.1. The DDE model (3.2.5) has a unique continuous solution defined for all $t \geq \tau$ and for a continuous initial condition.

Proof. Direct consequence of Theorems 3.1.1 and 3.1.2. 
Since we study a biological population, it is necessary for the system (3.2.5) to generate positive solutions.

Proposition 4.1.2. For nonnegative initial conditions, the solutions $Q(t)$ and $N(t)$ of (3.2.5) remain nonnegative for all $t \geq \tau$.

Proof. Claim 1: $Q(t) \geq 0, \quad \forall t \geq \tau$

Suppose by contradiction that $\exists t_{0}>\tau$ and $\exists \varepsilon>0$ such that $Q(t)>0$ for $t<t_{0}$, $Q\left(t_{0}\right)=0$, and $Q(t)<0$ for $t_{0} \in\left(t_{0}, t_{0}+\varepsilon\right)$. Evaluating the equation (3.2) at $t_{0}$ we get:

$$
\frac{d Q}{d t}\left(t_{0}\right)=2 e^{-\gamma_{S} \tau_{S}} \beta\left(Q\left(t_{0}-\tau_{S}\right)\right) Q\left(t_{0}-\tau_{S}\right) \geq 0 .
$$

At the same time,

$$
\frac{d Q}{d t}\left(t_{0}\right)=\lim _{\varepsilon \rightarrow 0} \frac{Q\left(t_{0}+\varepsilon\right)-Q\left(t_{0}\right)}{\varepsilon}=\lim _{\varepsilon \rightarrow 0} \frac{Q\left(t_{0}+\varepsilon\right)}{\varepsilon}<0 .
$$

Contradiction. Therefore $Q(t) \geq 0$ for all $t \geq \tau$.

Claim 2: $N(t) \geq 0, \quad \forall t \geq \tau$

Multiplying each term of equation (3.2) by $e^{\gamma_{N} t}$, we obtain:

$$
\frac{d}{d t}\left(N(t) e^{\gamma_{N} t}\right)=A_{N} e^{\gamma_{N} t} \kappa_{N}\left(N_{\tau_{N}}\right) Q_{\tau_{N}}
$$

Exactly as before, suppose by contradiction that $\exists t_{0}>\tau$ and $\exists \varepsilon>0$ such that $N(t)>0$ for $t<t_{0}, N\left(t_{0}\right)=0$, and $N(t)<0$ for $t_{0} \in\left(t_{0}, t_{0}+\varepsilon\right)$. For $t=t_{0}$,

$$
\left.\frac{d}{d t}\left(N(t) e^{\gamma_{N} t}\right)\right|_{t_{0}}=A_{N} e^{\gamma_{N} t} \kappa_{N}\left(N\left(t_{0}-\tau_{N}\right)\right) Q\left(t_{0}-\tau_{N}\right) \geq 0
$$

On the other hand,

$$
\left.\frac{d}{d t}\left(N(t) e^{\gamma_{N} t}\right)\right|_{t_{0}}=\lim _{\varepsilon \rightarrow 0} \frac{N\left(t_{0}+\varepsilon\right) e^{\gamma_{N}\left(t_{0}+\varepsilon\right)}-N\left(t_{0}\right) e^{\gamma_{N} t_{0}}}{\varepsilon}=\lim _{\varepsilon \rightarrow 0} \frac{N\left(t_{0}+\varepsilon\right) e^{\gamma_{N}\left(t_{0}+\varepsilon\right)}}{\varepsilon}<0
$$

which leads to a contradiction. Hence $N(t) \geq 0$ for all $t \geq \tau$. 


\subsection{Existence of steady states}

Mathematically, the steady states are obtained by setting the rates of change in (3.2.5) to zero:

$$
\frac{d Q}{d t}=\frac{d N}{d t}=\frac{d R}{d t}=\frac{d P}{d t}=0
$$

Therefore the steady state values are defined implicitly by the solutions of the following nonlinear system:

$$
\left\{\begin{aligned}
\beta\left(Q_{*}\right) Q_{*} & =\left\{-\left(\kappa_{N}\left(N_{*}\right)+\kappa_{P}+\kappa_{R}\left(R_{*}\right)\right)+2 e^{-\gamma_{S} \tau_{S}} \beta\left(Q_{*}\right)\right\} Q_{*} \\
\gamma_{N} N_{*} & =A_{N} \kappa_{N}\left(N_{*}\right) Q_{*} \\
\gamma_{R} R_{*} & =A_{R} \kappa_{R}\left(R_{*}\right)\left(1-e^{-\gamma_{R} \tau_{R S}}\right) Q_{*} \\
\gamma_{P} P_{*} & =\bar{A}_{P}^{*} \kappa_{P}\left(1-e^{-\gamma_{P} \tau_{P S}}\right) Q_{*}
\end{aligned}\right.
$$

Obviously, the trivial steady state $(0,0,0,0)$ is a solution but is not the most interesting equilibrium point because it corresponds biologically to the extinction of the cell population. We are interested only in positive solutions.

Remark 4. A necessary condition for the existence of positive solutions of (4.2.1) is $2 e^{-\gamma_{S} \tau_{S}}-1>0 \Leftrightarrow \gamma_{S} \tau_{S}<\ln 2$. Therefore, we will always work under this assumption.

Proposition 4.2.1. The nonlinear system (4.2.1) has a unique positive solution.

Proof. A simple calculation (see Appendix A) shows that the system (4.2.1) can be written as

$$
\left\{\begin{aligned}
a_{4} N_{*}\left(\theta_{1}^{n}+N_{*}^{n}\right) & =Q_{*} \\
a_{5} R_{*}\left(1+K_{r} R_{*}^{m}\right) & =Q_{*} \\
a_{8} N_{*}+a_{9} R_{*} & =Q_{*}\left(\frac{1}{\theta_{2}^{s}+Q_{*}^{s}}-a_{2}\right) \\
a_{6} P_{*} \exp \left(-\frac{a_{7}}{1+K_{P} P_{*}^{r}}\right) & =Q_{*}
\end{aligned}\right.
$$

where

$$
\begin{aligned}
a_{2} & =\frac{\kappa_{P}}{k_{0} \theta_{2}^{s}\left(2 e^{-\gamma_{S} \tau_{S}}-1\right)} \\
a_{4} & =\frac{\gamma_{N}}{f_{0} A_{N} \theta_{1}^{n}}
\end{aligned}
$$




$$
\begin{aligned}
a_{5} & =\frac{\gamma_{R}}{A_{R} \overline{k_{r}}\left(1-e^{-\gamma_{R} \tau_{R S}}\right)} \\
a_{6} & =\frac{\gamma_{P}}{A_{0} K_{P}\left(1-e^{-\gamma_{P} \tau_{P S}}\right)} \\
a_{7} & =\mu \tau_{P M} T_{\max } \\
a_{8} & =a_{4} \frac{f_{0} \theta_{1}^{n}}{k_{0} \theta_{2}^{s}\left(2 e^{-\gamma_{S} \tau_{S}}-1\right)}, \\
a_{9} & =a_{5} \frac{{\overline{\kappa_{r}}}_{k_{0} \theta_{2}^{s}\left(2 e^{-\gamma_{S} \tau_{S}}-1\right)}}{k} .
\end{aligned}
$$

The coefficients $a_{i}, i \in\{2,4,5,6,7,8,9\}$ are positive and depend explicitly on the delays.

Claim 1: The existence and the uniqueness of $Q_{*}$ imply the existence and uniqueness of all the other variables $P_{*}, R_{*}, N_{*}$.

Let

$$
\left\{\begin{aligned}
F_{1}\left(N_{*}\right) & :=a_{4} N_{*}\left(\theta_{1}^{n}+N_{*}^{n}\right) \\
F_{2}\left(R_{*}\right) & :=a_{5} R_{*}\left(1+K_{R} R_{*}^{m}\right) \\
F_{3}\left(Q_{*}\right) & :=Q_{*}\left(\frac{1}{\theta_{2}^{s}+Q_{*}^{s}}-a_{2}\right) \\
F_{4}\left(P_{*}\right) & :=a_{6} P_{*} \exp \left(-\frac{a_{7}}{1+K_{P} P_{*}^{r}}\right)
\end{aligned}\right.
$$

The functions $F_{1}, F_{2}, F_{4}$ are continuous differentiable, strictly increasing, convex, and positive, with the property that $F_{i}(0)=0, i \in\{1,2,4\}$. Therefore each of them has an inverse on $[0, \infty)$ with the same characteristics. Let $f_{i}:=F_{i}^{-1}, i \in\{1,2,4\}$. With the notation (4.2.3) the system (4.2.2) can be written as

$$
\left\{\begin{aligned}
N_{*} & =f_{1}\left(Q_{*}\right) \\
R_{*} & =f_{2}\left(Q_{*}\right) \\
a_{8} f_{1}\left(Q_{*}\right)+a_{9} f_{2}\left(Q_{*}\right) & =F_{3}\left(Q_{*}\right) \\
P_{*} & =f_{4}\left(Q_{*}\right)
\end{aligned}\right.
$$

The existence and the uniqueness of $Q_{*}$ is governed by the equation

$$
a_{8} f_{1}\left(Q_{*}\right)+a_{9} f_{2}\left(Q_{*}\right)=F_{3}\left(Q_{*}\right)
$$

For simplicity, let $G\left(Q_{*}\right):=a_{8} f_{1}\left(Q_{*}\right)+a_{9} f_{2}\left(Q_{*}\right)$. Besides differentiability, monotonicity (strictly increasing), and positivity, $\mathrm{G}$ has another important property. 


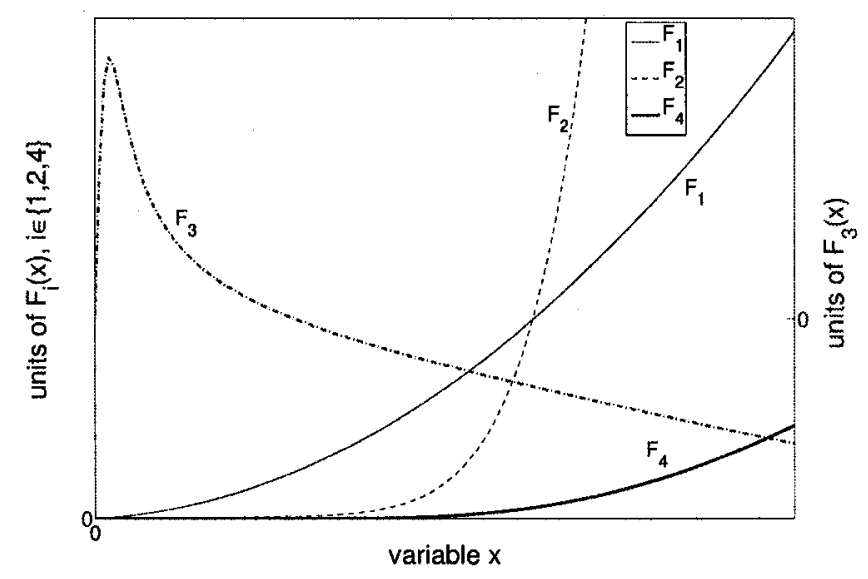

Fig. 4.1 The graphical representation of the functions $F_{i}, i=\overline{1,4}$ which corresponds to the steady state values of the coefficients (calculated using the numerical estimation from Table 3.1). Small perturbations from the equilibrium values do not change qualitatively the graphs of the functions.

Claim 2: $G$ is a concave function.

Indeed,

$$
\begin{aligned}
& f_{1}^{\prime}\left(Q_{*}\right)=\left(F_{1}^{-1}\right)^{\prime}\left(Q_{*}\right)=\frac{1}{F_{1}^{\prime}\left(N_{*}\right)} \\
& f_{2}^{\prime}\left(Q_{*}\right)=\left(F_{2}^{-1}\right)^{\prime}\left(Q_{*}\right)=\frac{1}{F_{2}^{\prime}\left(R_{*}\right)}
\end{aligned}
$$

which implies

$$
\begin{aligned}
& f_{1}^{\prime \prime}\left(Q_{*}\right)=\left(\frac{1}{F_{1}^{\prime}\left(N_{*}\right)}\right)^{\prime}=-\frac{F_{1}^{\prime \prime}\left(N_{*}\right)}{\left(F_{1}^{\prime}\left(N_{*}\right)\right)^{2}}<0 \\
& f_{2}^{\prime \prime}\left(Q_{*}\right)=\left(\frac{1}{F_{2}^{\prime}\left(R_{*}\right)}\right)^{\prime}=-\frac{F_{2}^{\prime \prime}\left(R_{*}\right)}{\left(F_{2}^{\prime}\left(R_{*}\right)\right)^{2}}<0
\end{aligned}
$$

Furthermore,

$$
G^{\prime \prime}\left(Q_{*}\right)=\underbrace{a_{8}}_{>0} f_{1}\left(Q_{*}\right)+\underbrace{a_{9}}_{>0} f_{2}\left(Q_{*}\right)<0
$$

Claim 3: The system (4.2.2) has a unique positive solution.

The solutions $Q_{*}$ of the system (4.2.2) are given by the intersection of the curves $G$ and $F_{3}$. Figure 4.2 captures the qualitative features of the curves described by the functions $F_{i}, i=\overline{1,4}$. Since $\mathrm{G}$ is a concave function, its graph intersects the graph of 
$F_{3}$ only in two distinct points: the origin and another positive point. Consequently, beside the trivial solution, the system (4.2.2) has a unique positive solution.

\subsection{Linear stability analysis}

The primary consideration of this section is the stability of the unique positive steady state defined by Proposition 4.2.1. Due to the nonlinearity of the equations, we cannot discuss this problem in its total generality. Rather, we examine the system behavior in a small neighborhood of the fixed point. The small perturbation assumption allows us to approximate the system (3.2.5) by a linear differential delay equation and to carry out a linear stability analysis.

\subsubsection{Basic mathematical concepts}

We maintain the notation introduced in Section 2.1. The delay differential equation $\dot{x}(t)=f\left(t, x_{\tau}(t)\right)$ with $f: \Omega \rightarrow \mathbb{R}^{n}$ and $\Omega \subset \mathbb{R} \times C$ was denoted (2.1.1).

Definition 4. A steady state of the system (3.1.1) is defined by the requirement that the solution $x$ is constant in time, i.e. $x(t)=x_{\tau}(t)=a$ constant denoted $x_{*}$ (steady state value).

Let $z(t):=x(t)-x_{*}$ be the deviation from the steady state. Therefore $z_{\tau}(t)=$ $x_{\tau}(t)-x_{*}$ and $\dot{z}(t)=\dot{x}(t)=f\left(t, x_{\tau}(t)\right)=f\left(t, z_{\tau}(t)+x_{*}\right)$. If we denote $g\left(t, z_{\tau}(t)\right):=$ $f\left(t, z_{\tau}(t)+x_{*}\right)$ then the system (3.1.1) is equivalent to

$$
\dot{z}(t)=g\left(t, z_{\tau}(t)\right)
$$

Definition 5. Assume that $g$ can be written as $g(t, \varphi)=L(t, \varphi)+F(t, \varphi)$, where $L$ is a continuous linear functional and $F$ has the property that $F(t, 0)=\frac{\partial F}{\partial \varphi}(t, 0)=0$. The system

$$
\dot{z}(t)=L\left(t, z_{\tau}(t)\right)
$$

is called the linearization of (4.3.1) about the trivial solution $z=0$. 
To analyze the stability of the null solution, we make the ansatz $z(t) \simeq a e^{\lambda t}$ with $a \in \mathbb{R}^{n}$. Substituting in (4.3.2) we notice that $a$ has a non-zero value only when

$$
\Delta(\lambda)=\operatorname{det}\left(\lambda \mathbf{I}-L\left(e^{\lambda t} \mathbf{I}\right)\right)=0
$$

Here $\mathbf{I}$ denotes the $n \times n$ identity matrix. Equation (4.3.3) is referred as the characteristic equation of the system (4.3.2) and its solutions are the eigenvalues of the system.

Definition 6. (Kuang (1993), Definition 4.1, p.25) We say that the solution $z=0$ of (4.3.2) is

(i) stable if $\forall \sigma \in \mathbb{R}, \forall \varepsilon \in \mathbb{R}, \exists \delta(\varepsilon, \sigma)$ such that

$$
\|\psi\| \leq \delta \Longrightarrow\left\|z_{\tau}(\sigma, \psi)(t)\right\| \leq \varepsilon, \forall t \geq \sigma
$$

Otherwise $z=0$ is called unstable.

(ii) asymptotically stable if it is stable and $\exists b_{0}=b_{0}(\sigma)$ such that

$$
\|\psi\| \leq b_{0} \Longrightarrow \lim _{t \rightarrow \infty}\left\|z_{\tau}(\sigma, \psi)(t)\right\|=0
$$

(iii) uniformly stable if $\forall \sigma \in \mathbb{R}, \forall \epsilon \in \mathbb{R}, \exists \delta(\epsilon)$ such that

$$
\|\psi\| \leq \delta \Longrightarrow\left\|z_{\tau}(\sigma, \psi)(t)\right\| \leq \varepsilon, \forall t \geq \sigma
$$

(iv) uniformly asymptotically stable if it is uniformly stable and $\exists b>0$ such that $\forall \eta, \exists t_{0}(\eta)$ such that

$$
\|\psi\| \leq b \Longrightarrow\left\|z_{\tau}(\sigma, \psi)(t)\right\| \leq \eta, \forall t \geq \sigma+t_{0}(\eta), \forall \sigma \in \mathbb{R}
$$

The most important stability results used in this chapter are concentrated in the following theorems (for complete proofs see (41) and (57)): 
Theorem 4.3.1. If $\sup \{\Re(\lambda) \mid \Delta(\lambda)=0\}<0$ then the zero solution of (4.3.2) is uniformly asymptotically stable. If $\Re(\lambda)>0$ for some eigenvalue $\lambda$ then $z=0$ is unstable. If $\Delta(\lambda)=0$ has a non-simple pure root then $z=0$ is unstable.

Theorem 4.3.2. If the null solution of (4.3.2) is uniformly asymptotically stable then the zero solution of (4.3.1) is also uniformly asymptotically stable. If $\Re(\lambda)>0$ for some $\lambda$ satisfying (4.3.3) then the zero solution of (4.3.1) is unstable.

In conclusion, carrying out the linear stability analysis of (3.1.1) is equivalent to determining conditions under which all the roots of the corresponding characteristic equation lie in the left half complex plane and are uniformly bounded away from the imaginary axis.

Remark 5. Let $\lambda=\mu+\imath \omega$ be a root of the characteristic equation (4.3.3).

(i) If $\mu=\Re(\lambda)<0$ then the solution decays and approaches zero in an oscillatory fashion.

(ii) If $\mu=\Re(\lambda)>0$ then the solution oscillates and diverges to infinity.

(iii) The boundary between these two situations $(\mu=\Re(\lambda)=0)$ defines a Hopf bifurcation, which is characterized by a pair of complex conjugated eigenvalues crossing the imaginary axis.

\subsubsection{Linearized system and characteristic equation}

In this subsection we write the linearization of the DDE model around the positive steady state $\left(Q_{*}, N_{*}, R_{*}, P_{*}\right)$ and derive the characteristic equation. The roots of this equation give insight into the stability of the system in a neighborhood of the fixed point.

Denote the right-hand side functions of the model equations (3.2.5) by

$$
\left\{\begin{aligned}
H_{1} & :=-\beta(Q) Q-\left(\kappa_{N}(N)+\kappa_{P}+\kappa_{R}(R)\right) Q+2 e^{-\gamma_{S} \tau_{S}} \beta\left(Q_{\tau_{S}}\right) Q_{\tau_{S}} \\
H_{2} & :=-\gamma_{N} N+A_{N} \kappa_{N}\left(N_{\tau_{N}}\right) Q_{\tau_{N}} \\
H_{3} & :=-\gamma_{R} R+A_{R}\left(\kappa_{R}\left(R_{\tau_{R M}}\right) Q_{\tau_{R M}}-e^{-\gamma_{R} \tau_{R S}} \kappa_{R}\left(R_{\tau_{R M}+\tau_{R S}}\right) Q_{\tau_{R M}+\tau_{R S}}\right) \\
H_{4} & :=-\gamma_{P} P+\bar{A}_{P} \kappa_{P}\left(Q_{\tau_{P M}}-e^{-\gamma_{P} \tau_{P S}} Q_{\tau_{P M}+\tau_{P S}}\right)
\end{aligned}\right.
$$


Since $H_{i}, i=\overline{1,4}$ are smooth functions we can write the expansion in Taylor series around $\left(Q_{*}, N_{*}, R_{*}, P_{*}\right)$;

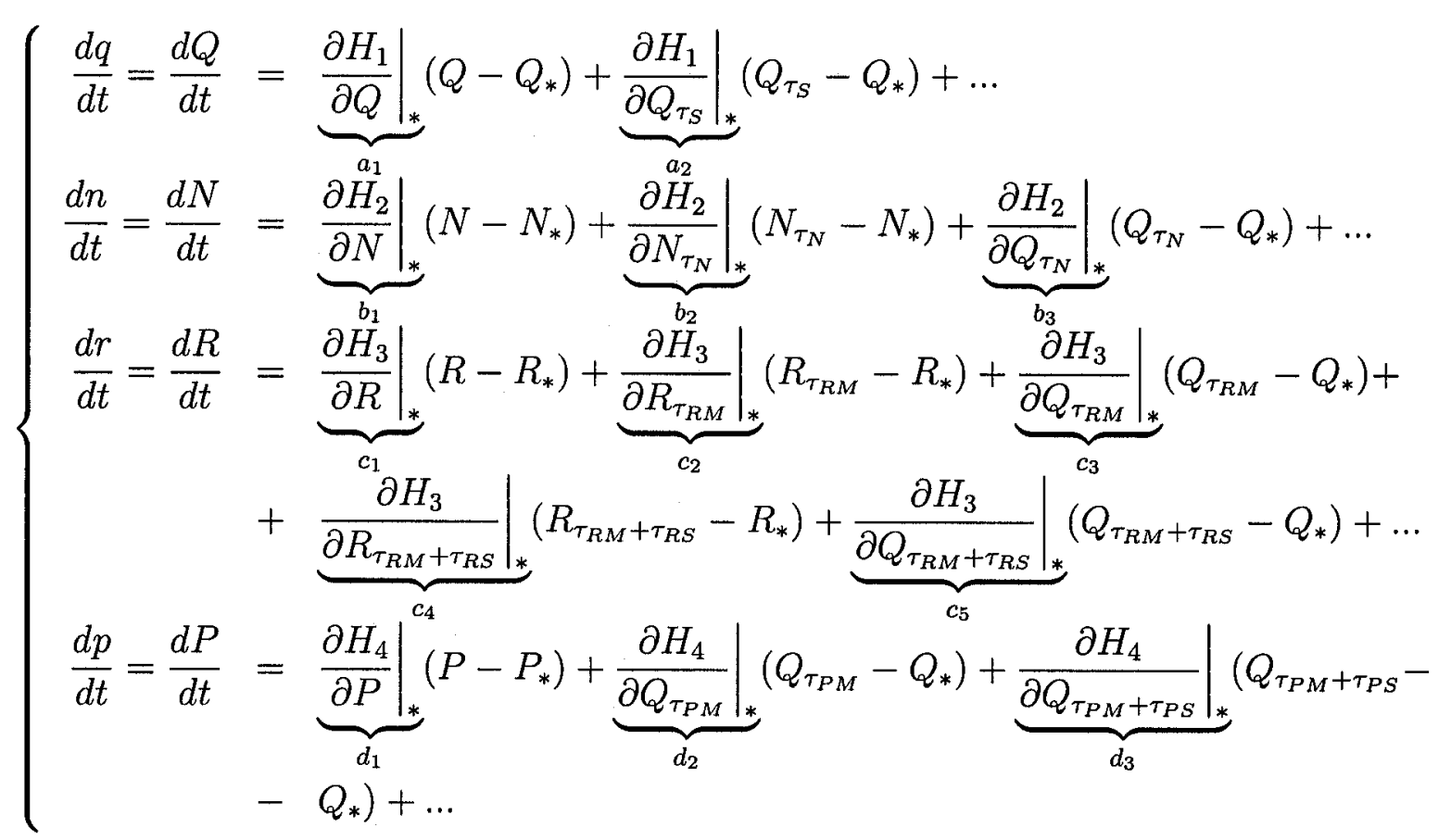

The notation $\left.\frac{d H}{d t}\right|_{*}$ refers to the evaluation at the steady state, i.e.

$$
\left.\frac{d H}{d t}\right|_{*}:=\frac{d H}{d t}\left(Q_{*}, N_{*}, R_{*}, P_{*}\right)
$$

Let $(q, n, r, p):=(Q, N, R, P)-\left(Q_{*}, N_{*}, R_{*}, P_{*}\right)$ be the new variables. Assuming that the deviation from the steady state is very small and, consequently, $(q, n, r, p)^{k}$ for any $k \geq 2$ is negligible, the linearized system can be written in the following form:

$$
\left\{\begin{array}{l}
\frac{d q}{d t}=a_{1} q+a_{2} q_{\tau_{S}} \\
\frac{d n}{d t}=b_{1} n+b_{2} n_{\tau_{N}}+b_{3} q_{\tau_{N}} \\
\frac{d r}{d t}=c_{1} r+c_{2} r_{\tau_{R M}}+c_{3} q_{\tau_{R M}}+c_{4} r_{\tau_{R M}+\tau_{R S}}+c_{5} q_{\tau_{R M}+\tau_{R S}} \\
\frac{d p}{d t}=d_{1} p+d_{2} q_{\tau_{P M}}+d_{3} q_{\tau_{P M}+\tau_{P S}}
\end{array}\right.
$$


Simple algebra gives the linearization coefficients:

$$
\left\{\begin{aligned}
a_{1} & =-\left(\beta\left(Q_{*}\right)+\kappa_{N}\left(N_{*}\right)+\kappa_{P}+\kappa_{R}\left(R_{*}\right)+\beta^{\prime}\left(Q_{*}\right) Q_{*}\right) \\
a_{2} & =2 e^{-\gamma_{S} \tau_{S}} \beta\left(Q_{*}\right) Q_{*}=a_{2}\left(\tau_{S}\right)>0 \\
b_{1} & =-\gamma_{N}<0 \\
b_{2} & =A_{N} \kappa_{N}^{\prime}\left(N_{*}\right) Q_{*}<0 \\
b_{3} & =A_{N} \kappa_{N}\left(N_{*}\right)>0 \\
c_{1} & =-\gamma_{R}<0 \\
c_{2} & =A_{R} \kappa_{R}^{\prime}\left(R_{*}\right) Q_{*}<0 \\
c_{3} & =A_{R} \kappa_{R}\left(R_{*}\right)>0 \\
c_{4} & =-A_{R} e^{-\gamma_{R} \tau_{R S}} \kappa_{R}^{\prime}\left(R_{*}\right) Q_{*}=c_{4}\left(\tau_{R S}\right)>0 \\
c_{5} & =-A_{R} e^{-\gamma_{R} \tau_{R S}} \kappa_{R}\left(R_{*}\right)=c_{5}\left(\tau_{R S}\right)<0 \\
d_{1} & =-\gamma_{P}-\mu A_{0} n_{P} \tau_{P M} K_{P} T_{m a x} Q_{*} \frac{P_{*}^{r-1}\left(1-e^{-\gamma_{P} \tau_{P S}}\right)}{\left(1+K_{P} P_{*}^{r}\right)^{2}} \\
& =d_{1}\left(\tau_{P S}, \tau_{P M}\right)<0 \\
d_{2} & =K_{P} A_{0} e^{\mu \tau_{P M} T_{*}}=d_{2}\left(\tau_{P M}\right)>0 \\
d_{3} & =-K_{P} A_{0} e^{\tau_{P M} T_{*}-\tau_{P S} \gamma_{P}}=d_{3}\left(\tau_{P S}, \tau_{P M}\right)<0
\end{aligned}\right.
$$

The system (4.3.5) can be expressed equivalently in the matrix form $\frac{d X}{d t}=A_{1} X(t)+A_{2} X\left(t-\tau_{S}\right)+A_{3} X\left(t-\tau_{N}\right)+A_{4} X\left(t-\tau_{R M}\right)+A_{5} X\left(t-\tau_{R M}-\right.$ $\left.\tau_{R S}\right)+A_{6} X\left(t-\tau_{P M}\right)+A_{7} X\left(t-\tau_{P M}-\tau_{P S}\right)$,

where

$$
\begin{gathered}
X(t)=\left[\begin{array}{c}
q(t) \\
n(t) \\
r(t) \\
p(t)
\end{array}\right], \quad A_{1}=\operatorname{diag}\left(a_{1}, b_{1}, c_{1}, d_{1}\right), \quad A_{2}=\left[\begin{array}{cccc}
a_{2} & 0 & 0 & 0 \\
0 & 0 & 0 & 0 \\
0 & 0 & 0 & 0 \\
0 & 0 & 0 & 0
\end{array}\right], \\
A_{3}=\left[\begin{array}{cccc}
0 & 0 & 0 & 0 \\
b_{3} & b_{2} & 0 & 0 \\
0 & 0 & 0 & 0 \\
0 & 0 & 0 & 0
\end{array}\right], \quad A_{4}=\left[\begin{array}{cccc}
0 & 0 & 0 & 0 \\
0 & 0 & 0 & 0 \\
c_{3} & 0 & c_{2} & 0 \\
0 & 0 & 0 & 0
\end{array}\right], \quad A_{5}=\left[\begin{array}{cccc}
0 & 0 & 0 & 0 \\
0 & 0 & 0 & 0 \\
c_{5} & 0 & c_{4} & 0 \\
0 & 0 & 0 & 0
\end{array}\right],
\end{gathered}
$$




$$
A_{6}=\left[\begin{array}{cccc}
0 & 0 & 0 & 0 \\
0 & 0 & 0 & 0 \\
0 & 0 & 0 & 0 \\
d_{2} & 0 & 0 & 0
\end{array}\right], \quad A_{7}=\left[\begin{array}{cccc}
0 & 0 & 0 & 0 \\
0 & 0 & 0 & 0 \\
0 & 0 & 0 & 0 \\
d_{3} & 0 & 0 & 0
\end{array}\right]
$$

Therefore the characteristic equation associated with the system (3.2.5) is given by $\operatorname{det}\left(\lambda I-A_{1}-A_{2} e^{\lambda \tau_{S}}-A_{3} e^{\lambda \tau_{N}}-A_{4} e^{\lambda \tau_{R M}}-A_{5} e^{\lambda\left(\tau_{R M}+\tau_{R S}\right)}-A_{6} e^{\lambda \tau_{P M}}-A_{7} e^{\lambda\left(\tau_{P M}+\tau_{P S}\right)}\right)=0$ with $\lambda \in \mathbb{C}$. After some calculations it reduces to

$$
\begin{aligned}
& \left(\lambda-a_{1}-a_{2}\left(\tau_{S}\right) e^{-\lambda \tau_{S}}\right)\left(\lambda-b_{1}-b_{2} e^{-\lambda \tau_{N}}\right)\left(\lambda-c_{1}-c_{2} e^{-\lambda \tau_{R M}}-c_{4} e^{-\lambda\left(\tau_{R M}+\tau_{R S}\right)}\right) \\
& \cdot\left(\lambda-d_{1}\right)=0
\end{aligned}
$$

\subsubsection{Subspaces of the stability region}

Following the discussion from Subsection 4.3.1, the stability of the trivial solution of (4.3.5) is governed by the real parts of the roots of the characteristic equation. Since equation (4.3.7) is a product of four transcendental equations the following result is immediate:

Lemma 4.3.3. All solutions of (4.3.7) have negative real parts if and only if all the roots of the transcendental equations

$$
\begin{aligned}
\lambda-a_{1}-a_{2}\left(\tau_{S}\right) e^{-\lambda \tau_{S}} & =0 \\
\lambda-b_{1}-b_{2} e^{-\lambda \tau_{N}} & =0 \\
\lambda-c_{1}-c_{2} e^{-\lambda \tau_{R M}}-c_{4}\left(\tau_{R S}\right) e^{-\lambda\left(\tau_{R M}+\tau_{R S}\right)} & =0 \\
\lambda-d_{1}\left(\tau_{P M}, \tau_{R S}\right) & =0
\end{aligned}
$$

have the same property.

Remark 6. (i) If all the delays are set to zero then the model (3.2.5) reduces to four ODE and the corresponding characteristic equation is reduced to a polynomial of degree four. In this case, the Fundamental Theorem of Algebra assures the existence of exactly four complex roots. 
(ii) In contrast to $O D E$ which require a finite number of points as initial conditions, the DDE necessitate an initial function defined on a time interval of length equal to the maximum delay. Consequently, the DDE model (3.2.5) is an infinite dimensional system.

(iii) Although a transcendental equation has an infinite set of solutions, only a finite number of roots have the real part greater than a given constant.

In the following subsections we analyze the transcendental equations from Lemma 4.3.3. The intersection of their stability regions delimitates the zone in the parameter space where the model displays stability.

\subsubsection{Analytical technique}

To our knowledge, the only analytical and geometrical criterion devoted to the stability of transcendental equations with delay dependent coefficients belongs to Berreta \& Kuang (10). Since their method is a powerful analytical tool in the next two subsections, we reproduce the main result.

Consider the general equation

$$
P_{n}(\lambda, \tau)+Q_{m}(\lambda, \tau) e^{-\lambda \tau}=0, \text { where }
$$

(a) $P_{n}(\lambda, \tau)=\sum_{k=0}^{n} p_{k}(\tau) \lambda^{k}$ and $Q_{m}(\lambda, \tau)=\sum_{k=0}^{m} q_{k}(\tau) \lambda^{k}$,

(b) $n>m$,

(c) $p_{k}(\cdot), q_{k}(\cdot): \mathbb{R}_{+} \rightarrow \mathbb{R}$ continuous and differentiable functions of $\tau$ with the property that $P_{n}(0, \tau)+Q_{m}(0, \tau)=p_{0}(\tau)+q_{0}(\tau) \neq 0, \forall \tau \in \mathbb{R}_{+}$.

We will assume further that the following conditions are satisfied:

(1) If $\lambda=\imath \omega, \omega \in \mathbb{R}$, then $P_{n}(\imath \omega, \tau)+Q_{m}(\imath \omega, \tau) \neq 0, \tau \in \mathbb{R}$;

(2) $\lim \sup \left\{\left|\frac{Q_{m}(\lambda, \tau)}{P_{n}(\lambda, \tau)}\right|:|\lambda| \longrightarrow \infty, \Re \lambda \geq 0\right\}<1$

(3) For each $\tau, F(\omega, \tau)=\left|P_{n}(\imath \omega, \tau)\right|^{2}-\left|Q_{m}(\imath \omega, \tau)\right|^{2}$ has at most a finite number of real zeros; 
(4) If a positive root $\omega(\tau)$ of $F(\omega, \tau)=0$ exists, then it is continuous and differentiable in $\tau$.

Remark 7. (i) $P_{n}(\lambda, \tau)$ are $Q_{m}(\lambda, \tau)$ functions analytic in $\lambda$ and differentiable in $\tau$.

(ii) The property $P_{n}(0, \tau)+Q_{m}(0, \tau)=p_{0}(\tau)+q_{0}(\tau) \neq 0, \forall \tau \in \mathbb{R}_{+}$is equivalent to $\lambda=0$ not a root of (4.3.8).

(iii) Condition (1) expresses that $P_{n}(\lambda, \tau)$ and $Q_{m}(\lambda, \tau)$ have no common imaginary roots.

(iv) Condition (2) ensures that no roots are bifurcating from infinity. Also, there are only finite "gates" for roots to cross the imaginary axis for a given $\tau$.

For simplicity, we will drop the indices $m$ and $n$.

Since for increasing $\tau$ the imaginary axis cannot be crossed by $\lambda(\tau)=0$ for some $\tau>0$, we look for the occurrence of a pair of simple conjugate imaginary roots $\lambda= \pm \imath \omega(\tau)$, with $\omega(\tau)$ real and positive, which crosses the imaginary axis at some positive $\tau^{*}$ value.

Note that $\overline{P(-\imath \omega, \tau)}=P(\imath \omega, \tau)$ and $\overline{Q(-\imath \omega, \tau)}=Q(\imath \omega, \tau)$ for all $\tau$ and $\omega$. Therefore, without loss of generality, we can consider $\lambda=\imath \omega(\tau)$, with $\omega(\tau)>0$. Substituting $\lambda=\imath \omega(\tau)$ in (4.3.8) we obtain that $\omega(\tau)$ must satisfy:

$$
\left\{\begin{array}{l}
\sin (\omega(\tau) \tau)=\frac{-\Re P(\imath \omega, \tau) \Im Q(\imath \omega, \tau)+\Im P(\imath \omega, \tau) \Re Q(\imath \omega, \tau)}{|Q(\imath \omega, \tau)|^{2}} \\
\cos (\omega(\tau) \tau)=-\frac{\Re P(\imath \omega, \tau) \Re Q(\imath \omega, \tau)+\Im P(\imath \omega, \tau) \Im Q(\imath \omega, \tau)}{|Q(\imath \omega, \tau)|^{2}}
\end{array}\right.
$$

$|Q(\imath \omega, \tau)|^{2} \neq 0$ because of the assumption (i).

Lemma 4.3.4. If $\omega(\tau)$ satisfies (4.3.9) then it is a root of

$$
F(\omega, \tau)=0
$$


Proof. Indeed,

$$
\left\{\begin{array}{l}
\sin (\omega(\tau) \tau)=\frac{-\Re P(\imath \omega, \tau) \Im Q(\imath \omega, \tau)+\Im P(\imath \omega, \tau) \Re Q(\imath \omega, \tau)}{|Q(\imath \omega, \tau)|^{2}}=\Im\left(\frac{P(\imath \omega, \tau)}{Q(\imath \omega, \tau)}\right) \\
\cos (\omega(\tau) \tau)=-\frac{\Re P(\imath \omega, \tau) \Re Q(\iota \omega, \tau)+\Im P(\imath \omega, \tau) \Im Q(\imath \omega, \tau)}{|Q(\imath \omega, \tau)|^{2}}=-\Re\left(\frac{P(\imath \omega, \tau)}{Q(\imath \omega, \tau)}\right)
\end{array}\right.
$$

Furthermore, $1=\sin (\omega(\tau) \tau)+\cos (\omega(\tau) \tau)=\left|\frac{P(\imath \omega, \tau)}{Q(\imath \omega, \tau)}\right|^{2} \Rightarrow|P(\imath \omega, \tau)|^{2}=|Q(\imath \omega, \tau)|^{2} \Rightarrow$ $F(\omega(\tau), \tau)=|P(\imath \omega, \tau)|^{2}-|Q(\imath \omega, \tau)|^{2}=0$.

Let $I$ be the set of all $\tau \geq 0$ defined by the following two conditions:

(a) if $\tau \in I$ then $\omega(\tau)$ is a positive root of $F(\omega, \tau)=0$,

(b) if $\tau \notin I$ then $\omega(\tau)$ does not satisfy (4.3.10) (i.e. we cannot have stability switches).

For any $\tau \in I, \omega(\tau)$ verifies (4.3.9). Therefore, we can define the angle $\theta(\tau) \in[0,2 \pi]$ as the solution of (4.3.9):

$$
\left\{\begin{array}{l}
\sin \theta(\tau)=\frac{-\Re P(\imath \omega, \tau) \Im Q(\imath \omega, \tau)+\Im P(\imath \omega, \tau) \Re Q(\imath \omega, \tau)}{|Q(\imath \omega, \tau)|^{2}} \\
\cos \theta(\tau)=-\frac{\Re P(\imath \omega, \tau) \Re Q(\imath \omega, \tau)+\Im P(\imath \omega, \tau) \Im Q(\imath \omega, \tau)}{|Q(\imath \omega, \tau)|^{2}}
\end{array}\right.
$$

Obviously, $\theta(\tau)=\omega(\tau) \tau+2 n \pi, n \in \mathbb{N}$. Hence, for any $n \in \mathbb{N}$ we can define the maps: $\tau_{n}: I \longrightarrow \mathbb{R}_{+}, \tau_{n}(\tau)=\frac{\theta(\tau)+2 n \pi}{\omega(\tau)}$ and $S_{n}: I \longrightarrow \mathbb{R}, S_{n}(\tau)=\tau-\tau_{n}(\tau)$.

Note that $S_{n}$ are continuous and differentiable on $I((10)$, Lemma 2.1, p. 1147).

The main result is the following:

Theorem 4.3.5. (Berreta and Kuang (2002, Theorem 2.2)) Assume that $\omega(\tau)$ is a a positive real root of (4.3.10) defined for $\tau \in I$, and at some $\tau^{*} \in I$, $S_{n}\left(\tau^{*}\right)=0$ for an $n \in \mathbb{N}$. Then:

(i) a pair of simple conjugate pure imaginary roots of (4.3.8) exists at $\tau^{*}$ :

$$
\lambda_{+}=\imath \omega\left(\tau^{*}\right) \text { and } \lambda_{-}=-\imath \omega\left(\tau^{*}\right) \text {. }
$$


(ii) the pair of imaginary roots $\left(\lambda_{+}, \lambda_{-}\right)$defined above crosses the imaginary axis from the left to the right if $\delta\left(\tau^{*}\right)>0$ and from the right to the left if $\delta\left(\tau^{*}\right)<0$, where

$$
\delta\left(\tau^{*}\right)=\operatorname{sign}\left\{\left.\frac{d \Re \lambda}{d \tau}\right|_{\lambda=\omega\left(\tau^{*}\right)}\right\}=\operatorname{sign}\left\{F_{\omega}^{\prime}\left(\omega\left(\tau^{*}\right) \tau^{*}\right)\right\} \operatorname{sign}\left\{\left.\frac{d S_{n}(\tau)}{d \tau}\right|_{\tau=\tau^{*}}\right\} .
$$

Motivation: The occurrence of characteristic roots crossing the imaginary axis from left to right implies that the nontrivial periodic solution near the steady state changes from local stability to being unstable.

\subsubsection{The equation $\lambda=A+B(\tau) e^{-\lambda \tau}$}

The transcendental equation

$$
\lambda=A+B(\tau) e^{-\lambda \tau}
$$

belongs to the general class $P_{n}(\lambda, \tau)+Q_{m}(\lambda, \tau) e^{-\lambda \tau}=0$, where $P_{n}, Q_{m}$ are polynomials in $\lambda$ with delay-dependent coefficients. Therefore we can apply Theorem 4.3.5 to obtain the following result:

Theorem 4.3.6. Consider the equation $\lambda=A+B(\tau) e^{-\lambda \tau} \quad$ (4.3.10) and assume that $|B(\tau)|>|A|$ and $A+B(\tau) \neq 0, \forall \tau \geq 0$. Then:

(i) The equation (4.3.11) has a pair of simple conjugate pure imaginary roots $\lambda_{+}\left(\tau_{*}\right)=\imath \omega\left(\tau_{*}\right), \lambda_{-}\left(\tau_{*}\right)=-\imath \omega\left(\tau_{*}\right)$, for $\omega\left(\tau_{*}\right)>0$ at $\tau^{*} \in I, S_{n}\left(\tau^{*}\right)=0$ for some $n \in \mathbb{N}_{0}$.

(ii) The pair $\left(\lambda_{+}, \lambda_{-}\right)$crosses the imaginary axis from the left to the right when $\delta\left(\tau^{*}\right)>0$ and from the right to the left when $\delta\left(\tau^{*}\right)<0$, where

$$
\delta\left(\tau^{*}\right):=\operatorname{sign}\left\{\omega \omega^{\prime}\left(-A+B^{2}(\tau) \tau\right)+\omega^{2} B^{2}(\omega)\right\} .
$$

Proof. In our case,

$$
\left\{\begin{array}{l}
P_{1}(\lambda, \tau)=\lambda-A \\
Q_{0}(\lambda, \tau)=-B(\tau)
\end{array}\right.
$$


with $p_{0}(\lambda)=-A, p_{1}(\lambda)=1$ and $q_{0}(\lambda)=-B(\tau)$. The assumption $A+B(\tau) \neq 0, \forall \tau \geq$ 0 ensures that $\lambda=0$ is not a root of (4.3.11). Hence a stability switch necessarily occurs when $\lambda= \pm \omega \omega, \omega>0$.

Let us check that the conditions (i)-(iv) imposed by Beretta \& Kuang (10) are satisfied. (i) and (ii) are immediate.

(iii) $F(\omega, \tau)=\left|P_{1}(\imath \omega, \tau)\right|^{2}-\left|Q_{0}(\imath \omega, \tau)\right|^{2}=A^{2}+\omega^{2}-B^{2}(\tau)$. For each $\tau \geq 0$, $F(\omega, \tau)=0$ gives the solution $\omega(\tau)= \pm \sqrt{B^{2}(\tau)-A^{2}}$ which are real if $|B(\tau)| \geq$ $|A|$.

(iv) We saw that, without loss of generality, we can take only the positive solution of $F(\omega, \tau)=0$. Therefore we choose $\omega(\tau)=\sqrt{B^{2}(\tau)-A^{2}}$ with $|B(\tau)| \geq|A|$. Obviously $\omega$, as a function of $\tau$, is continuous and differentiable.

Claim 1: The equation (4.3.11) has a pair of simple conjugate pure imaginary roots. For our particular transcendental equation $I=\{\tau \geq 0|| B(\tau)|>| A \mid\}$, and the angle $\theta(\tau) \in[0,2 \pi]$ is the solution of

$$
\left\{\begin{aligned}
\sin \theta(\tau) & =-\frac{\omega(\tau)}{B(\tau)} \\
\cos \theta(\tau) & =-\frac{A}{B(\tau)}
\end{aligned}\right.
$$

For any $n \in \mathbb{N}$ define the maps:

$$
\left\{\begin{aligned}
\tau_{n} & : I \longrightarrow \mathbb{R}_{+}, \tau_{n}(\tau)=\frac{\theta(\tau)+2 n \pi}{\omega(\tau)} \\
S_{n} & : I \longrightarrow \mathbb{R}, S_{n}(\tau)=\tau-\tau_{n}(\tau)
\end{aligned}\right. \text { and }
$$

The occurrence of stability switches takes place at zeros of the function $S_{n}(\tau)$. Assuming that exists $\tau^{*}$ such that $S_{n}\left(\tau^{*}\right)=0$ for some $n$, the equation (4.3.11) has a pair of simple conjugate pure imaginary roots $\lambda_{+}=\imath \omega\left(\tau^{*}\right)$ and $\lambda_{-}=-\imath \omega\left(\tau^{*}\right)$ (by Theorem 4.3.5).

Claim 2: $\delta\left(\tau^{*}\right)=\operatorname{sign}\left\{\omega \omega^{\prime}\left(-A+B^{2}(\tau) \tau\right)+\omega^{2} B^{2}(\omega)\right\}$

Using

$$
\operatorname{sign}\left\{\frac{d \Re \lambda}{d \tau}\right\}=\operatorname{sign}\left\{\Re\left(\frac{d \lambda}{d \tau}\right)^{-1}\right\}
$$


we can write

$$
\delta\left(\tau^{*}\right)=\operatorname{sign}\left\{\left.\frac{d \Re \lambda}{d \tau}\right|_{\lambda=\imath \omega\left(\tau_{*}\right)}\right\}=\operatorname{sign}\left\{\left.\Re\left(\frac{d \lambda}{d \tau}\right)^{-1}\right|_{\lambda=\imath \omega\left(\tau_{*}\right)}\right\}
$$

Consider $\lambda=\lambda(\tau)$ and differentiate the equation (4.3.11) with respect to $\tau$. We obtain

$$
\begin{aligned}
& \frac{d \lambda}{d \tau}=B^{\prime} e^{-\lambda \tau}-B\left(\lambda+\frac{d \lambda}{d \tau}\right) e^{-\lambda \tau} \\
&\left(\frac{d \lambda}{d \tau}\right)^{-1}=\frac{B}{\lambda-A}+B \tau \\
& B^{\prime}-B \lambda
\end{aligned}
$$

Therefore

$$
\begin{gathered}
\left.\left(\frac{d \lambda}{d \tau}\right)^{-1}\right|_{\lambda=\imath \omega}=\frac{\frac{B}{\imath \omega-A}+B \tau}{B^{\prime}-\imath B \omega}=\frac{\frac{-A+\imath \omega}{B}+B \tau}{B^{\prime}-\imath B \omega}= \\
=\frac{\left(-A+B^{2} \omega+\imath \omega\right)\left(B B^{\prime}+B^{2} \omega\right)}{\left(B B^{\prime}\right)^{2}+\left(B^{2} \omega\right)^{2}}=\frac{\left(-A+B^{2} \omega+\imath \omega\right)\left(\omega \omega^{\prime}+B^{2} \omega\right)}{\left(\omega \omega^{\prime \prime}\right)^{2}+\left(B^{2} \omega\right)^{2}}
\end{gathered}
$$

We have used that $\omega^{2}+A^{2}=B^{2}\left(\right.$ since $\omega(\tau)$ is a root of $\left.F(\omega, \tau)=A^{2}+\omega^{2}-B^{2}(\tau)\right)$ and $\omega \omega^{\prime}=B B^{\prime}$. Hence

$$
\left.\Re\left(\frac{d \lambda}{d \tau}\right)^{-1}\right|_{\lambda=\imath \omega\left(\tau_{*}\right)}=\frac{\omega \omega^{\prime}\left(-A+B^{2}(\tau) \tau\right)+\omega^{2} B^{2}(\omega)}{\left(\omega \omega^{\prime}\right)^{2}+\left(B^{2} \omega\right)^{2}}
$$

and

$$
\operatorname{sign}\left\{\left.\Re\left(\frac{d \lambda}{d \tau}\right)^{-1}\right|_{\lambda=\imath\left(\tau_{*}\right)}\right\}=\operatorname{sign}\left\{\omega \omega^{\prime}\left(-A+B^{2}(\tau) \tau\right)+\omega^{2} B^{2}(\omega)\right\}
$$




\subsubsection{The equation $\lambda=A+B e^{-\lambda \tau}$}

Hayes (40) was the first who provided a stability analysis of this type of equation. His work was continued and different methods of investigation can be found in Diekermann (23), Kolmanovski \& Nosov (53) and Stépán (90).

Theorem 4.3.7. Let

$$
\lambda=A+B e^{-\lambda \tau}
$$

with $A$ and $B$ real constants.

(i) The equation (4.3.13) has imaginary roots if $\left|\frac{A}{B}\right| \leq 1$.

(ii) All the roots of the equation (4.3.13) have negative real parts if $B \leq|A|$ and $\tau \leq \tau_{\text {crit }}=\frac{\arccos \left(-\frac{A}{B}\right)}{\sqrt{B^{2}-A^{2}}}$.

(iii) When $\tau=\tau_{\text {crit }}$ a Hopf bifurcation occurs with period $T_{H}=\frac{2 \pi}{\sqrt{B^{2}-A^{2}}}$.

Proof. Substituting $\lambda=\mu+\imath \omega$ in (4.3.13) and separating the real and the imaginary parts, we obtain:

$$
\begin{aligned}
\mu-A-B e^{-\mu} \cos (\omega \tau) & =0 \\
\omega+B e^{-\mu} \sin (\omega \tau) & =0 .
\end{aligned}
$$

The supremum of the real parts of the roots of the transcendental equation varies continuously with $\tau$. Therefore, if there is a transition from stability to instability, or the reverse, as $\tau$ varies, it must correspond to a purely imaginary root $\lambda=\imath \omega$. We notice that the above functions are even in $\omega$ (hence we can restrict our attention to $\omega \geq 0$ ) and they have singularities at $\omega=k \pi, k \in Z$.

(i) When $\mu=0$

$$
\begin{aligned}
& \omega+B \sin (\omega \tau)=0 \\
& A+B \cos (\omega \tau)=0
\end{aligned}
$$


which implies

$$
(B \cos (\omega \tau))^{2}+(B \sin (\omega \tau))^{2}=A^{2}+\omega^{2} \Rightarrow B^{2}=A^{2}+\omega^{2} \Rightarrow \omega=\sqrt{B^{2}-A^{2}}
$$

$\omega$ is real if $|B| \geq|A|$, i.e. $\left|\frac{A}{B}\right| \leq 1$.

(ii) Therefore, if $\lambda=\imath \omega$ is a solution of (4.3.13) then $\omega$ verifies (4.3.16) and (4.3.17). From (4.3.17) it follows that $\cos (\omega \tau)=-\frac{A}{B}$. Hence

$$
\tau=\frac{\arccos \left(-\frac{A}{B}\right)}{\omega}=\frac{\arccos \left(-\frac{A}{B}\right)}{\sqrt{B^{2}-A^{2}}} \quad \text { whenever } \quad\left|\frac{A}{B}\right| \leq 1 \text {. }
$$

Define $\tau_{\text {crit }}:=\frac{\arccos \left(-\frac{A}{B}\right)}{\omega}=\frac{\arccos \left(-\frac{A}{B}\right)}{\sqrt{B^{2}-A^{2}}}$.

Stability: A necessary (but not sufficient) condition in determining the boundary of the stability region is $A+B \leq 0$. We need to analyze individually two regions in the subspace defined by $A+B \leq 0$.

Claim 1: When the parameters $A$ and $B$ satisfy the conditions $A<0, B>0$, and $|A|>B$, all the roots of (4.3.13) have negative real parts.

For simplicity, consider the case when $\lambda$ is real. Since $B<0$, the right hand side of the equation (4.3.13) behaves like a decaying exponential with negative value $A+B<0$ at $\lambda=0$. Therefore it will intersect the diagonal $\lambda=\lambda$ at some negative point.

Claim 2: In the region defined by $B<0$ and $|A|>B$ the stability changes when crossing the boundary $\tau=\tau_{\text {crit }}$.

Indeed, in the region delimitated by $B<0$ and $|A|>B$, the stability can change only when crossing the boundaries $A=B$ and/or $\tau=\tau_{\text {crit }}$. When $A=B$ and $\tau=0, \lambda=A+B<0$. Moreover, on $A=B, \tau_{\text {crit }}=\frac{\arccos (-1)}{0}=\infty$, i.e. the stability will change at an infinite time. Since we had stability for $\tau=0$, we conclude that the solution remain stable. Therefore we have a stability switch only on the boundary $\tau=\tau_{\text {crit }}$.

To summarize, the equation (4.3.13) is stable when $B \leq|A|$ and $\tau \leq \tau_{\text {crit }}$. 


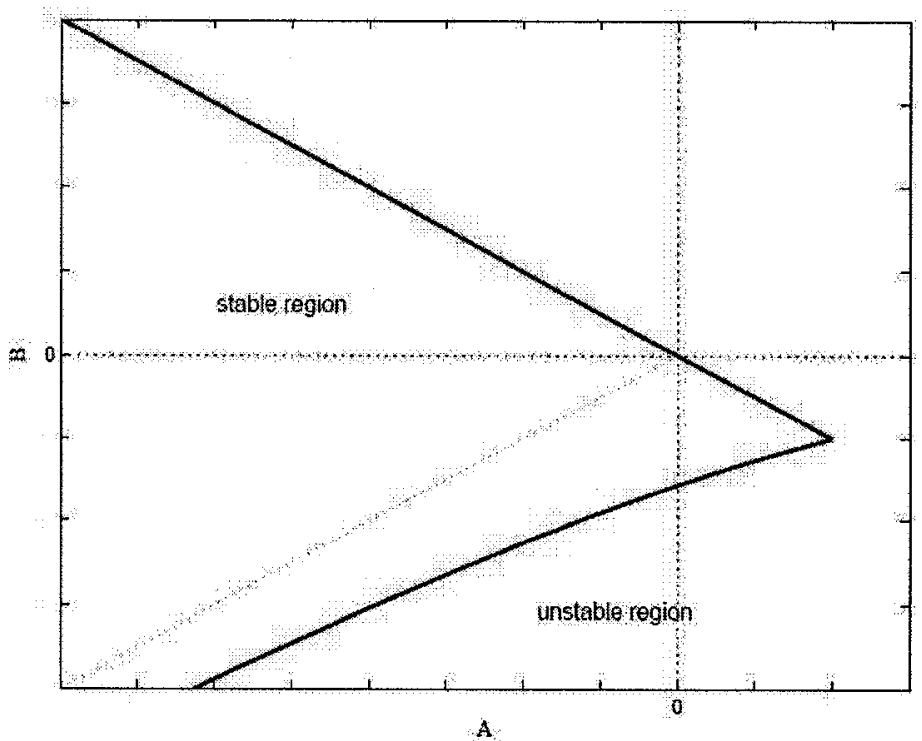

Fig. 4.2 Stability region bounded by the curves $\tau=\tau_{\text {crit }}$ and $A=-B$.

(ii) The Hopf period is $T_{H}=\frac{2 \pi}{\omega}=\frac{2 \pi}{\sqrt{B^{2}-A^{2}}}$.

Proposition 4.3.8. (i) If $A$ and $B$ have the same sign then $T_{H} \in\left[2 \tau_{\text {crit }}, 4 \tau_{\text {crit }}\right)$.

(ii) If $A$ and $B$ have different sign then $T_{H}>4 \tau_{\text {crit }}$.

Proof. $\tau_{\text {crit }}=\frac{\arccos \left(-\frac{A}{B}\right)}{\sqrt{B^{2}-A^{2}}} \Rightarrow \sqrt{B^{2}-A^{2}}=\frac{\arccos \left(-\frac{A}{B}\right)}{\tau_{\text {crit }}} \Rightarrow T_{H}=\frac{2 \pi \tau_{\text {crit }}}{\arccos \left(-\frac{A}{B}\right)}$.

(i) If $\mathrm{A}$ and $\mathrm{B}$ have the same sign then $\frac{A}{B}>0$ and $\arccos \left(-\frac{A}{B}\right) \in\left(\frac{\pi}{2}, \pi\right]$. Therefore $T_{H} \in\left[2 \tau_{\text {crit }}, 4 \tau_{\text {crit }}\right)$.

(ii) If $\mathrm{A}$ and $\mathrm{B}$ have different sign then $\frac{A}{B}<0$ and $\arccos \left(-\frac{A}{B}\right) \in\left[0, \frac{\pi}{2}\right)$. Therefore $T_{H}>4 \tau_{c r i t}$ 
Remark 8. This result is important for our numerical simulations because it shows that, for nonpositive coefficients, long period oscillations cannot occur.

Proposition 4.3.9. Let us assume that $A+B \neq 0$. The roots of $\lambda=A+B e^{-\lambda \tau}$ can cross the imaginary axis only from left to right as $\tau$ increases. If stability is lost at some critical value of $\tau$ (or does not exist for $\tau=0$ ), it can never be regained.

Proof. By Theorem 4.3.5 the sense of the stability switch is given by the sign of $\delta\left(\tau_{*}\right)$, where

$$
\delta\left(\tau^{*}\right)=\operatorname{sign}\left\{\left.\frac{d \Re \lambda}{d \tau}\right|_{\lambda=\imath \omega\left(\tau^{*}\right)}\right\}=\operatorname{sign}\left\{\left.\Re\left(\frac{d \lambda}{d \tau}\right)\right|_{\lambda=\omega \omega\left(\tau^{*}\right)}\right\}
$$

Consider $\lambda=\lambda(\tau)$ and differentiate equation (4.3.13) with respect to $\tau$ to obtain

$$
\frac{d \lambda}{d \tau}=-B \lambda e^{-\lambda \tau}-B e^{-\lambda \tau} \tau \frac{d \lambda}{d \tau} \Rightarrow \frac{d \lambda}{d \tau}=\frac{-B \lambda e^{-\lambda \tau}}{1+B \tau e^{-\lambda \tau}}
$$

When $\lambda=\imath \omega$,

$$
\begin{aligned}
\frac{d \lambda}{d \tau} & =\frac{-B \omega \omega e^{-\imath \tau}}{1+B \tau e^{-\imath \tau}} \\
\Re\left(\frac{d \lambda}{d \tau}\right) & =\frac{-B \omega \sin (\omega \tau)}{(1+B \tau \cos (\omega \tau))^{2}+(B \tau \sin (\omega \tau))^{2}}
\end{aligned}
$$

On the other hand, $B \cos (\omega \tau)=-A$ (by 4.3.17) and $B \sin (\omega \tau)=-\omega$ (by 4.3.16). Therefore

$$
\Re\left(\frac{d \lambda}{d \tau}\right)=\frac{\omega^{2}}{(1-A \tau)^{2}+\omega^{2}} \geq 0
$$

$\omega=0\left(\Leftrightarrow \Re\left(\frac{d \lambda}{d \tau}\right)=0\right)$ corresponds to $\lambda=0 \operatorname{root}$ of $(4.3 .13)$, i.e. $A+B=0$, which contradicts our assumption. Hence $\frac{d(\Re \lambda)}{d \tau}=\Re\left(\frac{d \lambda}{d \tau}\right)>0$ and the conclusion follows. 


\subsubsection{The equation $\lambda=A+B e^{-\lambda \tau_{1}}+C\left(\tau_{2}\right) e^{-\lambda\left(\tau_{1}+\tau_{2}\right)}$}

We resume the study of this type of two delay equation to the case required by our problem: $A<0, B<0, C\left(\tau_{2}\right)>0$.

Proposition 4.3.10. If $A+B+C\left(\tau_{2}\right)>0$ then the characteristic equation has a root with positive real part.

Proof. Let $f(\lambda)=\lambda-A-B e^{-\lambda \tau_{1}}-C\left(\tau_{2}\right) e^{-\lambda\left(\tau_{1}+\tau_{2}\right)}$. f is continuous differentiable with $f(0)=-A-B-C\left(\tau_{2}\right)<0$ and $\lim _{\lambda \rightarrow \infty} f(\lambda)=\infty$. Therefore, $f$ has a positive root for all $\tau_{1}, \tau_{2} \geq 0$.

Proposition 4.3.11. If $A+B+C\left(\tau_{2}\right)<0, A+B<C\left(\tau_{2}\right)$, and $0<1+B \tau_{1}$ $C\left(\tau_{2}\right) \cdot\left(\tau_{1}+\tau_{2}\right)$ then all the roots of $\lambda=A+B e^{-\lambda \tau_{1}}+C\left(\tau_{2}\right) e^{-\lambda\left(\tau_{1}+\tau_{2}\right)}$ have negative real parts.

Proof. Let $\lambda=\mu+\imath \omega \in \mathbb{C}$ be a root of

$$
\lambda=A+B e^{-\lambda \tau_{1}}+C\left(\tau_{2}\right) e^{-\lambda\left(\tau_{1}+\tau_{2}\right)}
$$

Separating the real and the imaginary parts in (4.3.18) we obtain:

$$
\left\{\begin{array}{r}
\mu-A-B e^{-\mu \tau_{1}} \cos \left(\omega \tau_{1}\right)-C e^{-\mu \tau_{2}} \cos \left(\omega\left(\tau_{1}+\tau_{2}\right)\right)=0 \\
\omega+B e^{-\mu \tau_{1}} \sin \left(\omega \tau_{1}\right)+C e^{-\mu \tau_{2}} \sin \left(\omega\left(\tau_{1}+\tau_{2}\right)\right)=0
\end{array}\right.
$$

Let $f(\lambda, \mu)=\mu-A-B e^{-\mu \tau_{1}} \cos \left(\omega \tau_{1}\right)-C e^{-\mu \tau_{2}} \cos \left(\omega\left(\tau_{1}+\tau_{2}\right)\right)$. Since $\cos (x) \in[-1,1]$ for all $x$, it follows that $f(\lambda, \mu) \geq \underbrace{\mu-A-B e^{-\mu \tau_{1}}+C e^{-\mu\left(\tau_{1}+\tau_{2}\right)}}_{g(\mu)}$.

Claim 1: $g$ is an increasing function.

$$
\begin{aligned}
g^{\prime}(\mu) & =1+B \tau_{1} e^{\mu \tau_{1}}-C\left(\tau_{2}\right) \cdot\left(\tau_{1}+\tau_{2}\right) e^{-\mu\left(\tau_{1}+\tau_{2}\right)} \\
g^{\prime \prime}(\mu) & =-B \tau_{1}^{2}+C\left(\tau_{2}\right) \cdot\left(\tau_{1}+\tau_{2}\right)^{2} e^{-\mu\left(\tau_{1}+\tau_{2}\right)}
\end{aligned}
$$

$B<0, C>0 \Longrightarrow g^{\prime \prime}(\mu)>0$ for all $\mu \Longrightarrow g^{\prime}$ is increasing. Hence

$$
0<\underbrace{g^{\prime}(0)}_{1+B \tau_{1}-C\left(\tau_{1}+\tau_{2}\right)}<g^{\prime}(\mu) \leq \lim _{\mu \rightarrow \infty} g^{\prime}(\mu)=\infty \text { for all } \mu \geq 0
$$


which implies that $g$ is an increasing function on $[0, \infty)$.

Claim 2: All the roots of (4.3.18) have negative real parts.

Since $g$ is an increasing function on $[0, \infty)$ it follows that

$$
g(\mu) \geq g(0)=-\left(A+B+C\left(\tau_{2}\right)\right)>0 \text { for all } \mu \geq 0 .
$$

Let $\lambda=\mu+\imath \omega \in \mathbb{C}$ be a root of (4.3.18). Then $f(\lambda, \mu)=0$. But whenever $\mu \geq 0$, $f(\lambda, \mu) \geq g(\mu) \geq g(0)=-\left(A+B+C\left(\tau_{2}\right)\right)>0$. Therefore $\mu<0$.

\subsubsection{Conclusions}

The primary consideration of this subsection is to identify some regions in the parameter space where the model (3.2.5) displays stability or unstability. Performing a linear stability analysis around the unique positive steady state $\left(Q_{*}, N_{*}, R_{*}, P_{*}\right)$ we found the characteristic equation as a product of four transcendental equations:

$$
\begin{aligned}
& \left(\lambda-a_{1}-a_{2}\left(\tau_{S}\right) e^{-\lambda \tau_{S}}\right)\left(\lambda-b_{1}-b_{2} e^{-\lambda \tau_{N}}\right)\left(\lambda-c_{1}-c_{2} e^{-\lambda \tau_{R M}}-c_{4} e^{-\lambda\left(\tau_{R M}+\tau_{R S}\right)}\right) . \\
& \cdot\left(\lambda-d_{1}\right)=0 .
\end{aligned}
$$

First we summarize the properties discussed in the previous subsections for each of the equations involved in the characteristic equation. We confine our study to the particular case of the coefficients defined by (4.3.6). Their positivity/nonpositivity plays an important role and simplifies our study: $a_{2}, c_{4}>0$ and $b_{1}, b_{2}, c_{1}, c_{2}, d_{1}<0$.

Lemma 4.3.12. The equation $\lambda-b_{1}-b_{2} e^{-\lambda \tau_{N}}=0$ with $b_{1}<0, b_{2}<0$ is stable for $\tau_{N} \leq \tau_{\text {crit }}=\frac{\arccos \left(-\frac{b_{1}}{b_{2}}\right)}{\sqrt{b_{2}^{2}-b_{1}^{2}}}$ and unstable otherwise. A Hopf bifurcation occurs when $\tau=\tau_{\text {crit }}$ with period $T_{H}=\frac{2 \pi}{\sqrt{b_{2}^{2}-b_{1}^{2}}}$.

Proof. Immediate application of Theorem 4.3.7 for $A=b_{1}, B=b_{2}$.

Lemma 4.3.13. Assume that the coefficients of the equation $\lambda-a_{1}-a_{2}\left(\tau_{S}\right) e^{-\lambda \tau_{S}}=0$ satisfy $a_{1}+a_{2}(\tau) \neq 0$ for all $\tau \geq 0$. A pair of simple complex conjugate pure imaginary 
roots $\lambda= \pm \imath \omega(\tau)$ can occur only when $0<\tau<\tau_{1}:=\frac{\ln \frac{1}{\left|a_{1}\right|}}{2 \gamma_{S} Q_{*} \beta\left(Q_{*}\right)}$. No stability changes can happen when $\tau \geq \tau_{1}$.

Proof. This equation is a particular case of the problem discussed in Theorem 4.3.6 for $A=a_{1}, B=a_{2}, \tau=\tau_{S}$. The hypothesis $a_{1}+a_{2}(\tau) \neq 0, \forall \tau \geq 0$ ensures that $\lambda=0$ is not a root of the transcendental equation. Therefore a stability switch necessarily occurs when $\lambda= \pm \imath \omega(\tau)$ with $\omega(\tau)>0$ solution of $F(\omega, \tau)=a_{1}^{2}+\omega^{2}-a_{2}^{2}(\tau)$. Precisely, $\omega(\tau)=\sqrt{a_{2}^{2}(\tau)-a_{1}^{2}}$, which is real whenever $\left|a_{2}(\tau)\right|>\left|a_{1}\right|$, i.e. $\tau<\tau_{1}:=$ $\frac{\ln \frac{1}{\left|a_{1}\right|}}{2 \gamma_{S} Q_{*} \beta\left(Q_{*}\right)}$.

Lemma 4.3.14. Consider the transcendental equation $\lambda-c_{1}-c_{2} e^{-\lambda \tau_{R M}}-c_{4}\left(\tau_{R S}\right)$. $\cdot e^{-\lambda\left(\tau_{R M}+\tau_{R S}\right)}=0$.

(i) If $c_{1}+c_{2}+c_{4}\left(\tau_{R S}\right)>0$ then the above equation has a root with positive real part.

(ii) If $c_{1}+c_{2}+c_{4}\left(\tau_{R S}\right)<0, c_{1}+c_{2}<c_{4}\left(\tau_{R S}\right)$, and $1+c_{2} \tau_{R M}-c_{4}\left(\tau_{R S}\right) \cdot\left(\tau_{R M}+\tau_{R S}\right)>0$ then all the roots of the above equation have negative real parts.

Proof. Direct consequence of Propositions 4.3.10 and 4.3.11 for $A=c_{1}, B=c_{2}$, $C=c_{4}, \tau_{1}=\tau_{R M}$, and $\tau_{2}=\tau_{R S}$.

Lemma 4.3.15. All the roots of the equation $\lambda-d_{1}\left(\tau_{P_{M}}, \tau_{R S}\right)=0$ have negative real parts.

Proof. Since $d_{1}\left(\tau_{P M}, \tau_{R S}\right)<0$ for all $\tau_{P M}, \tau_{R S}$ positive, the conclusion follows immediately.

All these properties lead to the next result:

Proposition 4.3.16. If either one of the following conditions

(i) $c_{1}+c_{2}+c_{4}\left(\tau_{R S}\right)>0$

(ii) $\tau_{N}>\tau_{c r i t}=\frac{\arccos \left(-\frac{b_{1}}{b_{2}}\right)}{\sqrt{b_{2}^{2}-b_{1}^{2}}}$ 
is satisfied then the positive steady state $\left(Q_{*}, N_{*}, R_{*}, P_{*}\right)$ of the system (3.2.5) is unstable.

Proof. The stability of the steady state $\left(Q_{*}, N_{*}, R_{*}, P_{*}\right)$ is governed by the roots of the characteristic equation (4.3.7). Since all the roots of have negative real parts, the analysis is reduced to the study of equations:

$$
\begin{aligned}
\lambda-a_{1}-a_{2}\left(\tau_{S}\right) e^{-\lambda \tau_{S}} & =0 \\
\lambda-b_{1}-b_{2} e^{-\lambda \tau_{N}} & =0 \\
\lambda-c_{1}-c_{2} e^{-\lambda \tau_{R M}}-c_{4}\left(\tau_{R S}\right) e^{-\lambda\left(\tau_{R M}+\tau_{R S}\right)} & =0
\end{aligned}
$$

(i) When $c_{1}+c_{2}+c_{4}\left(\tau_{R S}\right)>0$ the equation $\lambda-c_{1}-c_{2} e^{-\lambda \tau_{R M}}-c_{4}\left(\tau_{R S}\right) e^{-\lambda\left(\tau_{R M}+\tau_{R S}\right)}=$ 0 has a root with positive real part (by Lemma 4.3.14). Therefore the steady state $\left(Q_{*}, N_{*}, R_{*}, P_{*}\right)$ is unstable.

(ii) The region in the parameter space delimited by $b_{1}<0, b_{2}<0$, and $\tau_{N}>\tau_{c r i t}$ is of unstability for equation $\lambda-b_{1}-b_{2} e^{-\lambda \tau_{N}}=0$ (by Lemma 4.3.12), and consequently, for the characteristic equation of the model. 


\section{Chapter 5}

\section{Simulating Cyclical Thrombocytopenia}

The focus of this chapter is to investigate the mechanism underlying the platelet fluctuations in cyclical thrombocytopenia using the mathematical model of hematopoiesis (3.2.5). First, we want to understand the model dynamics by looking at the influence of each parameter on the stability of the steady state, and by analyzing the changes in the amplitude and the period of oscillations once stability is lost. Periodic hematological diseases like PCML and CN, which involve fluctuations in all blood cell lines, are believed to arise in the stem cell compartment in the bone marrow. Since all the reported cases of CT, except for Füreder et al. (36) and Menitove et al. (70), reveal that besides oscillations in platelet count the patient's hematological profile has been consistently normal, a destabilization of a peripheral control mechanism might play an important role in the genesis of this disorder. For this reason we confine our numerical investigation to the set of parameters which generate periodic solutions in the platelet compartment and keep all the other model variables at their steady state values. The next step of our investigation is to fit the simulated data provided by the model to the published platelet counts (see Figures 2.4, 2.5, 2.6, 2.7, $2.8,2.9,2.10,2.11$ ). For this purpose we choose the simulated annealing algorithm (78), having as starting point in the parameter space the conclusion drawn from the previous numerical analysis. To increase the understanding of pathogenesis of cycli- 
cal thrombocytopenia, a few research groups $((15),(51),(100))$ have measured the platelet levels as well as the thrombopoietin concentrations in their patients. The model duplicates not only the platelet cyclical patterns seen in cyclical thrombocytopenia but also the reported TPO levels. The hypothesis for the origin of platelet oscillations based on the model predictions is consistent with the clinical findings and may help to improve therapeutic strategies.

Numerical Software: The delay differential equations comprising the model (3.2.5) are numerically integrated using a Runge-Kutta method (time step 0.05 days) incorporated in the mathematical software XPP (30). The initial conditions are the steady state values from Table 3.1 and approximately 200 days are discarded to eliminate transient behavior (see Appendix C for the XPP code). All the other experiments are performed with Matlab.

\subsection{Parameters influence on the model dynamics}

Initially, we tried numerical experiments based on the existing notions of the mecha-

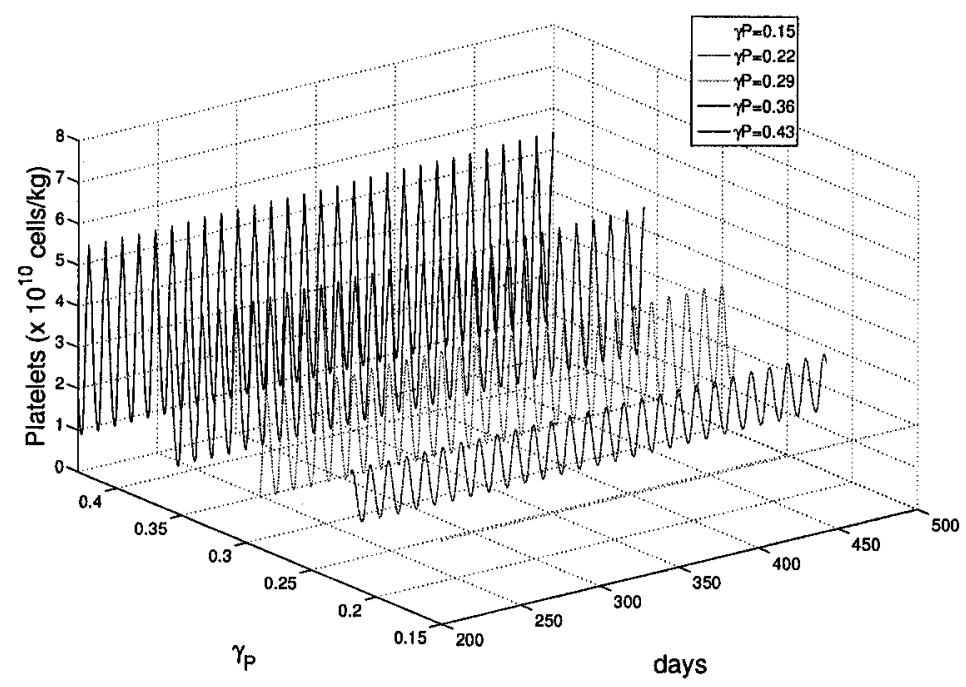

Fig. 5.1 Highly sensitive model response to an increased death rate of circulating platelets $\gamma_{P}$. All the other parameter values are taken from Table 3.1. 


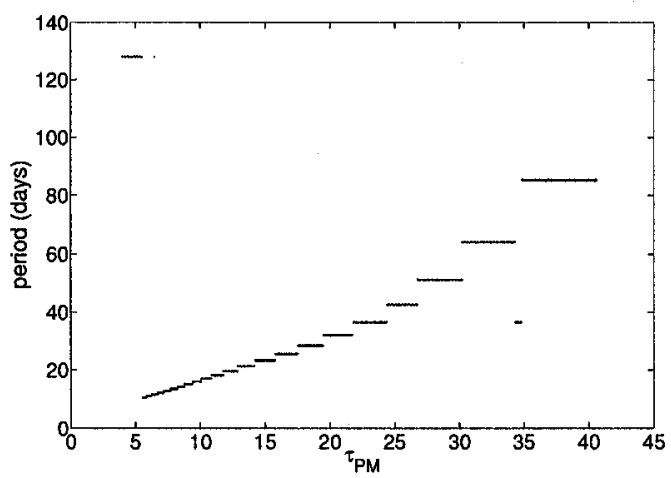

Fig. 5.2 Evolution of periodicity in platelet oscillations when the maturation time of megakaryocytes $\tau_{P M}$ is modified.

nisms of cyclical thrombocytopenia. The literature up to now explains the aetiology of this disease in terms of platelet destruction (autoimmune CT) or cyclical failure in platelet production (amegakaryocytic CT). Spectral data analysis revealed that an important feature of CT is the significant periods of platelet fluctuations. They vary between individuals and are in the range 13-64 days. Firstly, we increase the death rate of circulating platelets $\gamma_{P}$ to determine whether this change induces oscillations like those seen in autoimmune data. Although the model displays a highly sensitive response to small changes in $\gamma_{P}$ and the platelet counts start immediately to oscillate (Figure 5.1), the period of fluctuations remains unchanged ( $\simeq 13$ days). It is of interest to discover how the mathematical model can generate oscillatory solutions with different periodicity. Repeated numerical integrations show that the main parameter controlling the period of platelet fluctuations is the maturation time of megakaryocytes $\tau_{P M}$. Figure 5.2 captures the period evolution when $\tau_{P M}$ is varied over a large range. As it is illustrated in Figures 5.3, 5.4, and 5.5, changes in $\gamma_{P}$ or $\mu$ do not significantly affect the period of platelet oscillations. Based on the hypothesis that the cyclical patterns in amegakaryocytic CT are secondary to the failure in platelet production, we decreased the platelet differentiation rate $\kappa_{P}$ from its steady state value to much lower levels. This change did not lead to a bifurcation in the stability of the steady state, and sustained oscillations in the platelet compartment.

Cyclical thrombocytopenia involves either thrombocytosis (high platelet count), 

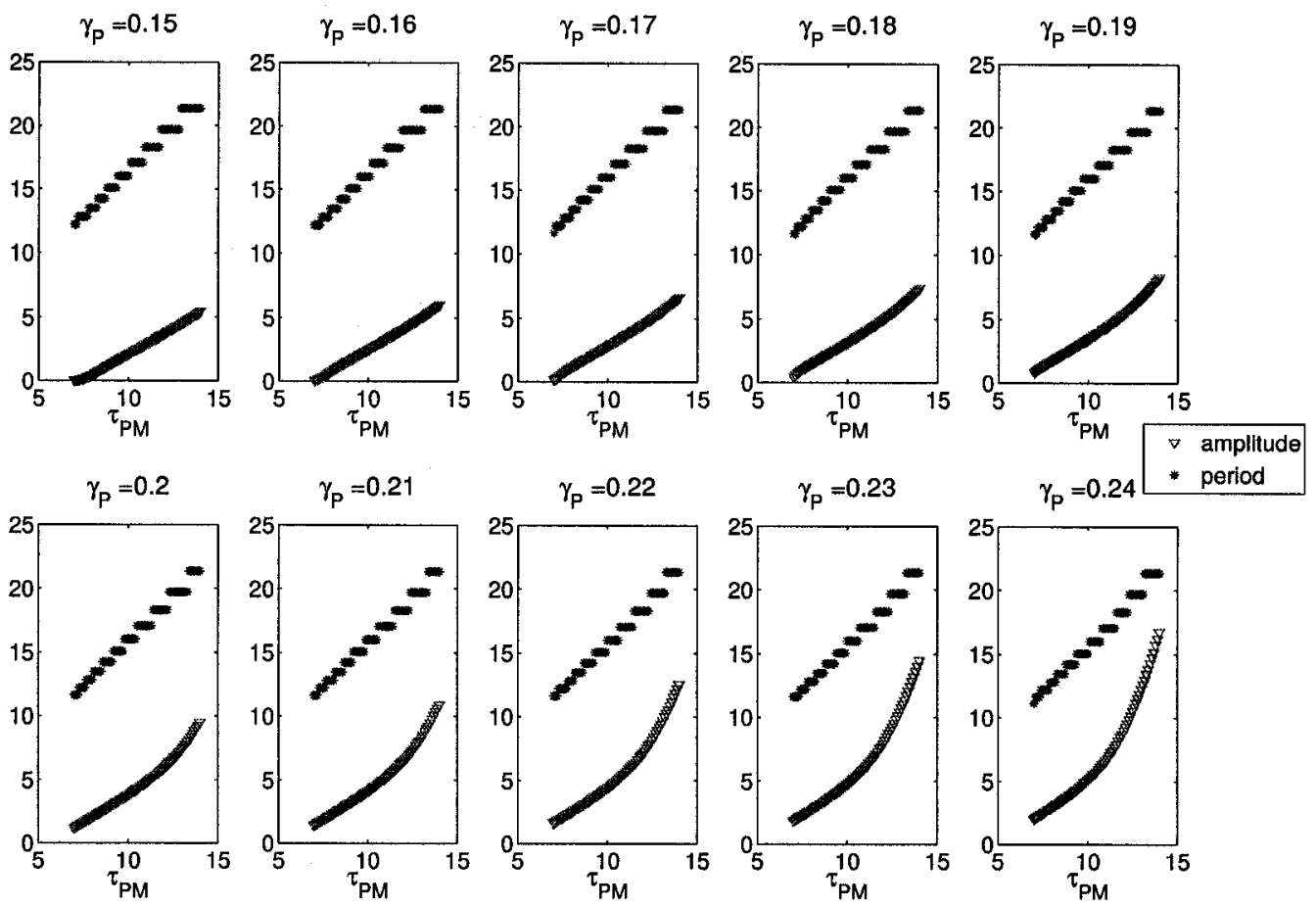

Fig. 5.3 Changes in the period (days) and the amplitude $\left(\times 10^{10}\right.$ cells $/ \mathrm{kg}$ ) of platelet fluctuations when $\gamma_{P}$ and $\tau_{P M}$ are varied simultaneously. All the other parameters are kept at their normal values (see Table 3.1). A small increase in $\gamma_{P}$ determines an oscillatory response with an increased amplitude but unchanged period. Similarly, perturbations in $\tau_{P M}$ values induce platelet oscillations with variable period and unchanged amplitude.

thrombocytopenia (low platelet values) or an alternation of both. One way to simulate the low/high platelet levels is by decreasing/increasing the minimal number of platelets released per megakaryocyte $\left(A_{0}\right)$ and the effective growth rate of megakaryocytes $(\mu)$.

The above discussion identifies a subset in the parameter space with an essential role in generating a model response similar with the CT platelet data: the platelet apoptosis rate $\left(\gamma_{P}\right)$, the effective growth rate of megakaryocytes $(\mu)$, the minimal number of platelets released per megakaryocyte $\left(A_{0}\right)$, and the megakaryocytes matu- 


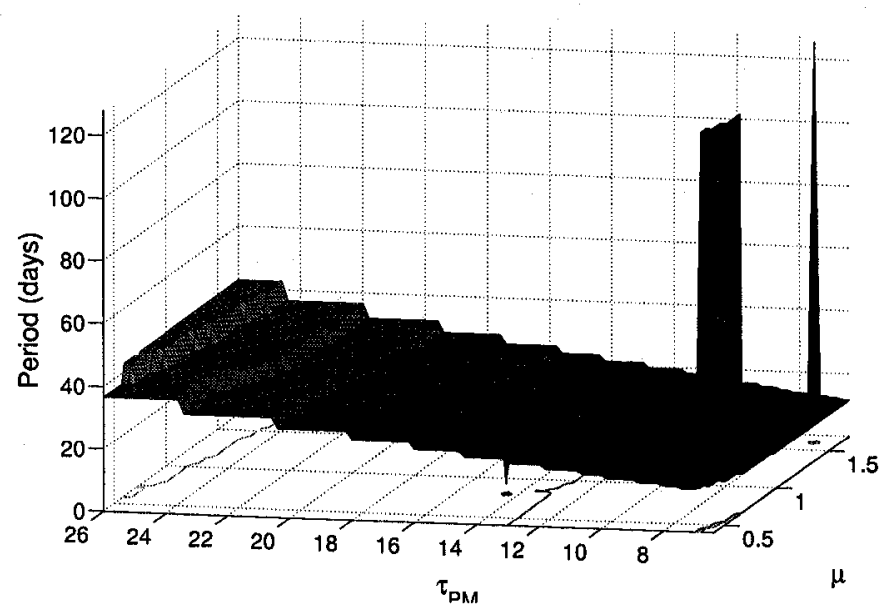

Fig. 5.4 Period evolution of platelet oscillations when $\tau_{P M}$ and $\mu$ are varied simultaneously. All the other parameters are kept at their normal values given in Table 3.1. Changes in $\mu$ do not significantly affect the periodicity.

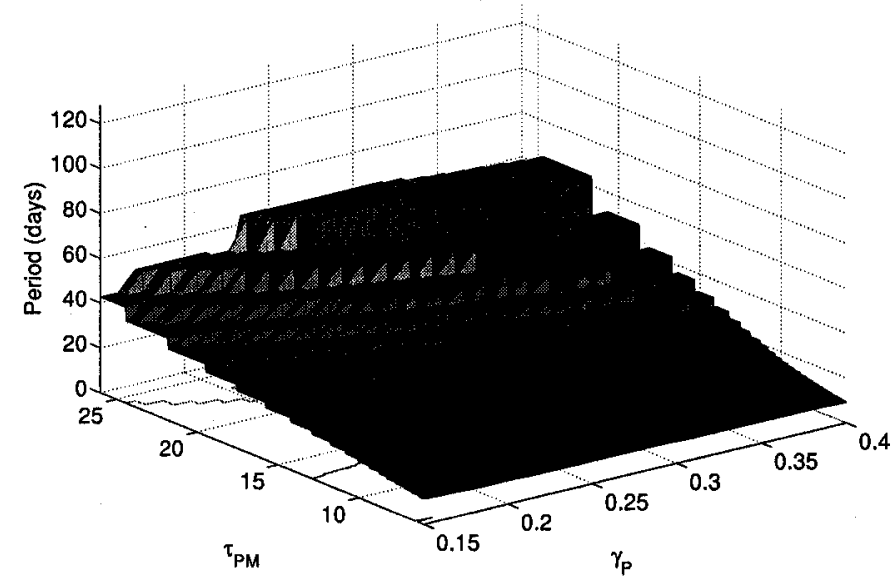

Fig. 5.5 Period evolution of platelet oscillations when $\gamma_{P}$ and $\tau_{P M}$ are varied simultaneously. All the other parameters are kept at their normal values given in Table 3.1. Changes in $\gamma_{P}$ do not significantly affect the periodicity. 


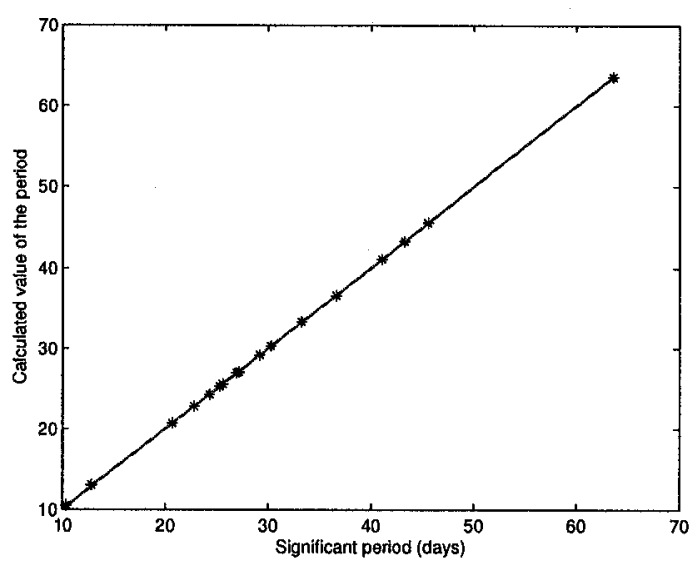

Fig. 5.6 The significant periods found in reported cases of CT are linear combinations of the model delays.

ration time $\left(\tau_{P M}\right)$. None of the changes in the values of these four parameters perturb the stem cells, erythrocytes or neutrophils normal levels.

Given the relevance of the periodicity in the investigation of CT we are interested to discover the potential connections with the cell cycle duration. We fit the function

$$
F\left(n_{1}, n_{2}, n_{3}, n_{4}, n_{5}, n_{6}\right)=n_{1} \tau_{S}+n_{2} \tau_{N}+n_{3} \tau_{R M}+n_{4} \tau_{R S}+n_{5} \tau_{P M}+n_{6} \tau_{P S}
$$

with $n_{1}, n_{2}, n_{3}, n_{4}, n_{5}, n_{6}$ nonnegative integers, to the set of the significant periods found in CT patients. Each period can be expressed as a linear combination of the stem cell proliferation time $\left(\tau_{S}\right)$, erythrocytes maturation time $\left(\tau_{R M}\right)$, megakaryocytes maturation time $\left(\tau_{P M}\right)$ and aging time of platelets $\left(\tau_{P S}\right)$. The reader is referred to Table 5.1 and Figure 5.6 for details.

\subsection{Simulated annealing method: description and advantages}

An important type of optimization problem is to find the "best" configuration of a system which can exist in a very large number of possible states. For example, of considerable interest to the chemical and solid state communities is to find the lowest energy configurations of thousands of molecules. The interpretation of the echoes of 


\begin{tabular}{cccccccccc}
\hline $\begin{array}{c}\text { Significant period } P \\
\text { (days) }\end{array}$ & $n_{1}$ & $n_{2}$ & $n_{3}$ & $n_{4}$ & $n_{5}$ & $n_{6}$ & $\begin{array}{c}\text { calculated value } \\
\text { of the period } \bar{P}\end{array}$ & $\begin{array}{c}\text { error } \\
(P-\bar{P})\end{array}$ \\
\hline Autoimmune CT & & & & & & & & \\
\hline 24.3256 & 1 & 0 & 2 & 0 & 0 & 1 & 24.3 & 0.0256 \\
12.8284 & 0 & 0 & 1 & 0 & 1 & 0 & 13 & 0.1716 \\
25.3133 & 1 & 0 & 1 & 0 & 1 & 1 & 25.3 & 0.0133 \\
20.7125 & 4 & 0 & 0 & 0 & 0 & 1 & 20.7 & 0.0125 \\
25.6095 & 2 & 0 & 1 & 0 & 2 & 0 & 25.6 & 0.0095 \\
45.5809 & 2 & 0 & 0 & 0 & 3 & 2 & 45.6 & 0.0191 \\
10.3564 & 0 & 1 & 0 & 0 & 1 & 0 & 10.5 & 0.1436 \\
27.1555 & 2 & 0 & 2 & 0 & 0 & 1 & 27.1 & 0.0555 \\
\hline Amegakaryocytic CT & & & & & & & & \\
\hline 63.5809 & 2 & 0 & 1 & 0 & 2 & 4 & 63.6 & 0.0191 \\
41.0863 & 2 & 0 & 0 & 0 & 1 & 3 & 41.1 & 0.0137 \\
43.2957 & 1 & 0 & 1 & 0 & 2 & 0 & 43.3 & 0.0290 \\
26.9331 & 0 & 0 & 1 & 0 & 3 & 0 & 27 & 0.0669 \\
22.8290 & 0 & 0 & 1 & 0 & 3 & 0 & 22.8 & 0.0669 \\
30.2663 & 1 & 0 & 3 & 0 & 0 & 1 & 30.3 & 0.0337 \\
29.2065 & 4 & 0 & 3 & 0 & 0 & 0 & 29.2 & 0.0065 \\
27.1946 & 2 & 0 & 2 & 0 & 0 & 1 & 27.1 & 0.0946 \\
33.2723 & 1 & 0 & 0 & 0 & 3 & 1 & 33.3 & 0.0277 \\
36.6502 & 2 & 0 & 2 & 0 & 0 & 2 & 36.6 & 0.0502 \\
27.0000 & 0 & 0 & 1 & 0 & 3 & 0 & 27 & 0.0000 \\
\hline \hline
\end{tabular}

Table 5.1 The significant periods found in cyclical thrombocytopenia patients are linear combinations of the model delays. For each case we identify the best combination of coefficients $n_{i}, i \in \overline{\{1,6\}}$, calculate $\bar{P}=$ $n_{1} \tau_{S}+n_{2} \tau_{N}+n_{3} \tau_{R M}+n_{4} \tau_{R S}+n_{5} \tau_{P M}+n_{6} \tau_{P S}$ and estimate the error $P-\bar{P}$. The errors are very small and vary in the range $[0,0.1716]$.

an explosion received by an receptor on the earth surface is another application of industrial significance.

Up to now, simulated annealing proved to be one of the most successful techniques for the large scale optimization problems where a desired global minimum is hidden among many local extrema. This method was introduced by S. Kirkpatrick, C. D. Gelatt and M. P. Vecchi in 1983 (52), and since then it effectively solved many practical problems. Two relevant examples are minimizing the travel distance for a salesman such that he will not have to visit the same city more than once and designating integrated circuits with million of elements placed such that the interference between the connected wires is minimized.

The name of and the inspiration behind this method come from thermal annealing. At high temperatures the molecules of a liquid move freely with respect to another. As the temperature is reduced, the thermal mobility is lost and the atoms tend to crystallize into a solid. If the sample is quenched (cooled quickly), then usually 
the solid ends up in a polycrystalline or amorphous state. If the sample is annealed (cooled slowly) then an ample time is allowed for atoms to redistribute, increasing the chance of forming a perfect crystal. Actually, the crystal is the state with minimum energy of the system.

Inspired by the physical processes, Kirkpatrick et al. (52) introduce a global temperature parameter $\mathcal{T}$ to control the cooling rate of the system. The success of the method depends on the annealing schedule (the sequence of decreasing temperatures). The cooling schedule is not unique and it is a real challenge to find the appropriate one for each problem. For example, we would expect it to depend on the energy landscape. If some local minima are separated from the global minimum by deep valleys, then the algorithm needs a slower rate of cooling. Neal (75) noticed that the most commonly used is the geometric schedule, i.e. $\mathcal{T}(t+1)=\alpha \mathcal{T}(t)$, with $0<\alpha<1$. Some optimization problems are better solved with adaptive annealing schedules, where $\mathcal{T}(t+1)$ depends on the states of the system visited up to time $t+1$.

We need four ingredients to define the simulated annealing problem:

(i) a description of the system configurations,

(ii) a random generator of state rearrangements,

(iii) an objective (or energy) function,

(iv) an annealing (or cooling) schedule.

The algorithm works by simulating a random walk on the set of the configuration space that searches for low energy states. At each instant during the simulation we have a current state from which we randomly select a neighbor and consider whether or not to move at the new configuration and try again.

The outline of a single simulated annealing run is as follows:

(1) Randomly generate a solution array for the problem and compute its cost $\left(E_{0}\right)$. They are the best-so-far values.

(2) Set the temperature to the initial value. 
(3) Run a Metropolis Monte Carlo simulation at this temperature by repeating the following steps:

(a) Generate a new solution and calculate its energy $\left(E_{1}\right)$.

(b) Determine if the new configuration is accepted or rejected.

$\left(b_{1}\right)$ If $E_{1}<E_{0}$ the new solution is accepted and used as a starting point for the next step. The new configuration and its energy become the best-so-far values.

$\left(b_{2}\right)$ The case $E_{1}>E_{0}$ is treated probabilistically. The acceptance probability is given by $P=\exp \left(-\frac{E_{1}-E_{0}}{\mathcal{T}}\right)$. Generate random numbers uniformly distributed in the interval $(0,1)$. One of this numbers is selected and compared to $P$. The new configuration is accepted only if this number is less than $P$.

(4) Reduce the temperature by choosing the next value given by the cooling schedule. If the new value of the temperature is greater or equal to the final fixed temperature then return to Step 3. Otherwise the run is finished and the bestso-far values are stored and reported.

The final effective temperature should be large enough such that the simulation is still able to visit all "energetically" reasonable solutions. Otherwise the run might be trapped into a local minimum.

Interestingly enough, a method known as simulated quenching, which involves the rapid decrease of temperature, is successfully used for optimization problems arising in biology or chemistry.

\subsection{Fitting the model simulations to the platelet data}

For our optimization problem we choose as an energy function the pointwise least square approximation between the clinical data and simulated platelet data provided 
by the mathematical model:

$$
E=\sqrt{\sum_{i=1}^{M} \frac{\left(P_{i}^{s}-P_{i}\right)^{2}}{\bar{P}^{2}}}
$$

$M$ is the number of points available, the superscript $s$ indicates simulated data and the lack of a superscript denotes CT data. Also, the bar indicates that mean was taken and the presence of $\bar{P}$ provides scaling information. We minimize the square root of the pointwise least square because it deforms the function monotonically, making the energy landscape less steep. Figure 5.7 shows a typical 3D representation of the objective function.

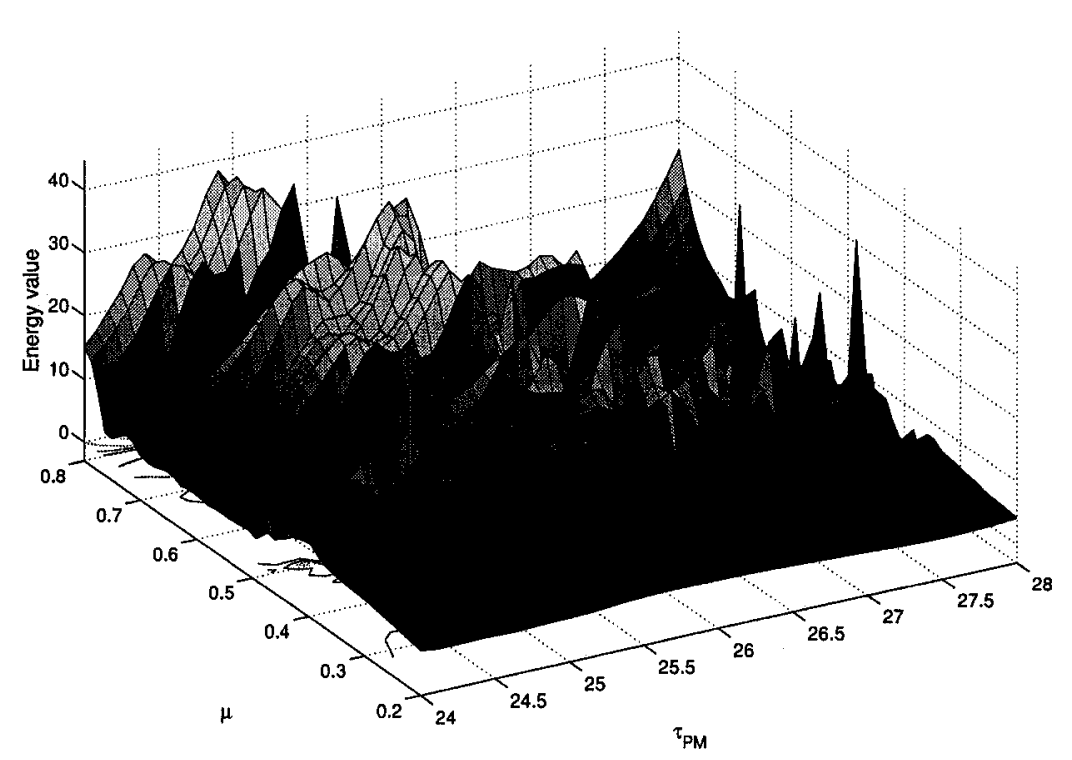

Fig. 5.7 3D representation of the energy function constructed with Yanabu et al. (99) data in a small part of the parameter space. The simulated annealing algorithm finds the minimum energy state when $\gamma_{P}=0.2738, \quad \tau_{P M}=17.001, \quad \mu=0.23746$.

Initially, we tried the geometric cooling schedule with $\alpha$ very close to 1 ( $\alpha=0.999)$ but, for our situation, it was not slow enough to find the "optimum" configuration. 
Although not very common, the constant thermodynamic speed schedule allows sufficient time for finding the minimum energy state:

$$
\Delta \mathcal{T}=-\frac{v \mathcal{T}^{2}}{\varepsilon \sigma(E)}, \quad \text { where }
$$

$\Delta \mathcal{T}=$ difference between current and previous temperature,

$\sigma(E)=$ standard deviation of current energy,

$\varepsilon=$ estimate of relaxation time,

$v=$ thermodynamic speed (constant).

We applied this approach to the CT data, comparing the model simulation to the platelet counts published in the literature. By varying the parameters $\gamma_{P}, \mu, \tau_{P M}$, $A_{0}$, and, in some isolated cases, $\kappa_{P}$ and $\theta_{2}$, we were able to successfully duplicate the dynamic clinical features of CT. The simulated annealing output and platelet data recorded from the patients diagnosed with CT are presented in Figures 5.8, 5.9, 5.10, 5.11. The left-hand panels contain the sampled simulation (model output sampled at the same time points as the clinical data) and the right-hand column shows the full platelet simulation generated by our model. Since the available experimental results associate autoimmune CT to an immune-mediated platelet destruction, we would expect that an increase in the rate of platelet clearance $\left(\gamma_{P}\right)$ would be the primary change necessary for duplicating some cases of CT. Indeed, $\gamma_{P}$ appears to be involved in fitting autoimmune data as well as in few amegakaryocytic situations, with significantly increased values in the first variety of the disease (average 0.3 with a standard deviation of 0.09) comparable to the latter (average 0.19 with a standard deviation of 0.01 ).

The platelet differentiation rate $\left(\kappa_{P}\right)$, the minimal number of platelets released per megakaryocyte $\left(A_{0}\right)$, and the relative growth rate of megakaryocyte $(\mu)$ were changed while fitting the model to platelet data, as it was hypothesized that amegakaryocytic CT involves a megakaryocyte deficiency and a cyclical failure in platelet production. Repeated numerical experiments indicate that there is no significant advantage varying $\kappa_{P}$. In all cases but one (100), the fitting algorithm did not modify this parameter. Surprisingly, for the patient reported by (100) a stem cell parameter change 


\begin{tabular}{|c|c|c|c|c|c|c|c|}
\hline Source and patient ID & $\gamma P$ & $\tau_{P M}$ & $\mu$ & $\overline{A_{0}}$ & $\overline{\theta_{2}}$ & $\kappa_{P}$ & transient \\
\hline Normal values & 0.15 & 7 & 1.7836 & 0.1 & 0.095 & 0.028 & \\
\hline Autoimmune CT & $\uparrow$ & $\uparrow$ & & $\downarrow$ & & & \\
\hline Kosugi et al. & 0.40317 & 16.995 & 0.044527 & 0.056734 & NV & NV & 200 \\
\hline Yanabu et al. & 0.2738 & 17.001 & 0.23746 & NV & NV & NV & 212 \\
\hline Rocha et al. & 0.35764 & 14.0086 & 0.16654 & NV & NV & NV & 200 \\
\hline Kimura et al. & 0.2 & 26 & 0.17836 & NV & NV & NV & 216 \\
\hline Skoog et al. & 0.21923 & 20.0024 & 0.17913 & 0.016788 & NV & NV & 203.5 \\
\hline Ámegakaryocytic CT & $\uparrow$ & $\uparrow$ & $\downarrow$ & & & & \\
\hline Bruin et al. & $\mathrm{NV}$ & 16.598 & 0.200644 & 0.00050384 & NV & NV & 215 \\
\hline Zent et al. & NV & 19.656 & 0.1482 & NV & 0.05435 & 0.002332 & 198 \\
\hline Hoffman et al. & NV & 40.044 & 0.048642 & NV & NV & NV & 205 \\
\hline Wilkinson and Firkin & 0.157238 & 27.839 & 0.21415 & NV & NV & NV & 205 \\
\hline Engstrom et al. & 0.19614 & 27.4434 & 0.17548 & NV & NV & NV & 205 \\
\hline Aranda and Dorantes & 0.20632 & 19.297 & 0.15719 & NV & NV & NV & 218 \\
\hline Wasastjerna & 0.39526 & 16.374 & 0.17911 & NV & NV & NV & 222 \\
\hline \multicolumn{8}{|l|}{ C-TPO } \\
\hline Lewis & 0.22 & 18 & 0.17836 & 0.01 & NV & $\overline{\mathrm{NV}}$ & 215 \\
\hline
\end{tabular}

Table 5.2 Parameter estimates for the CT patients based on simulated annealing method. NV stands for the normal value taken from Table 3.1. $\uparrow$ and $\downarrow$ indicate the qualitative parameter change relative to the normal value.

was necessary for fitting. Although it was sufficient to change $\tau_{P M}, \mu$, and $\kappa_{P}$ to the numerical values from Table 5.2 to mimic the platelet counts of this patient, stem cell levels increased to approximately $2.2 \times 10^{6}$ cells $/ \mathrm{kg}$. When reducing $\theta_{2}$ at about half of its normal value, stem cells returned to the equilibrium, while the platelet counts remained unchanged. In the simulated annealing results, the values of $\mu$ are drastically reduced relative to the normal value of 1.7839 ( $\mathrm{U}$ days $/ \mathrm{kg})^{-1} . \mu$ varied between $1 / 45$ and $1 / 10$ of the steady state value which is physiologically equivalent to a slower relative growth of megakaryocytes in all CT patients investigated in our study. These findings are in agreement with the clinical features of CT. Bruin et al. (15) and Zent et al. (100) described amegakaryocytic patients with small megakaryocytes in the ascending limb of the platelet count cycle (see Figure 5.13). Only in a few cases was it necessary to decrease $A_{0}$ to simulate CT. Generally, this parameter was reduced to either half or $1 / 10$ of its normal value. Interestingly, in a single case of amegakaryocytic CT (15) $A_{0}$ was decreased by a factor of 200 compared to the steady state value. This is consistent with the clinical observations, since bone marrow aspirates in this patient showed small megakaryocytes with no release of platelets (15).

Since the period of platelet fluctuations in CT data varies between individuals, 
and $\tau_{P M}$ (megakaryocyte maturation time) is the parameter with the most prominent influence on the platelet oscillations generated by the model, we allowed $\tau_{P M}$ to vary. What was not anticipated in this study is the major role played by $\tau_{P M}$ in reproducing the oscillatory patterns observed in CT. Furuyama et al. (37) presented an autoimmune case where the megakaryocytes are always abundant, but most of them are immature at platelet nadir and only 50\% are mature at the time of high platelet count. Also, Aranda and Dorantes (3) noticed that in their patient CT was related to a periodic variation in maturity of megakaryocytes. Our results indicate that megakaryocytes need about twice as much time as normal to maturate in autoimmune CT. In the patients with amegakaryocytic CT, which have on average longer periods of platelet fluctuations, $\tau_{P M}$ is elevated between two and six times the normal value.
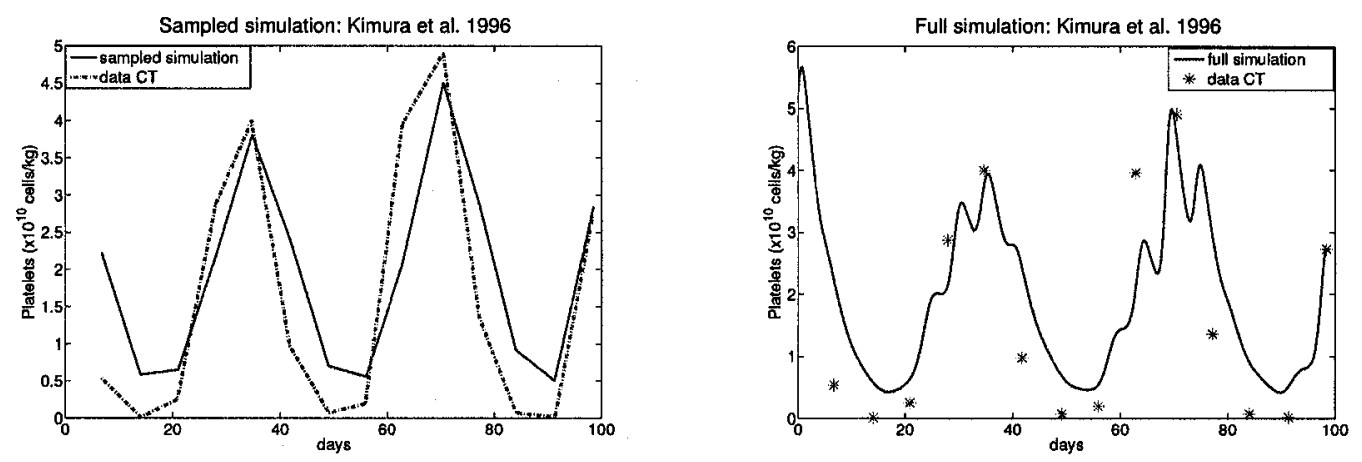

Fig. 5.8 Output of the simulated annealing fitting and the published platelet data. Sampled (left) and full (right) simulation. 

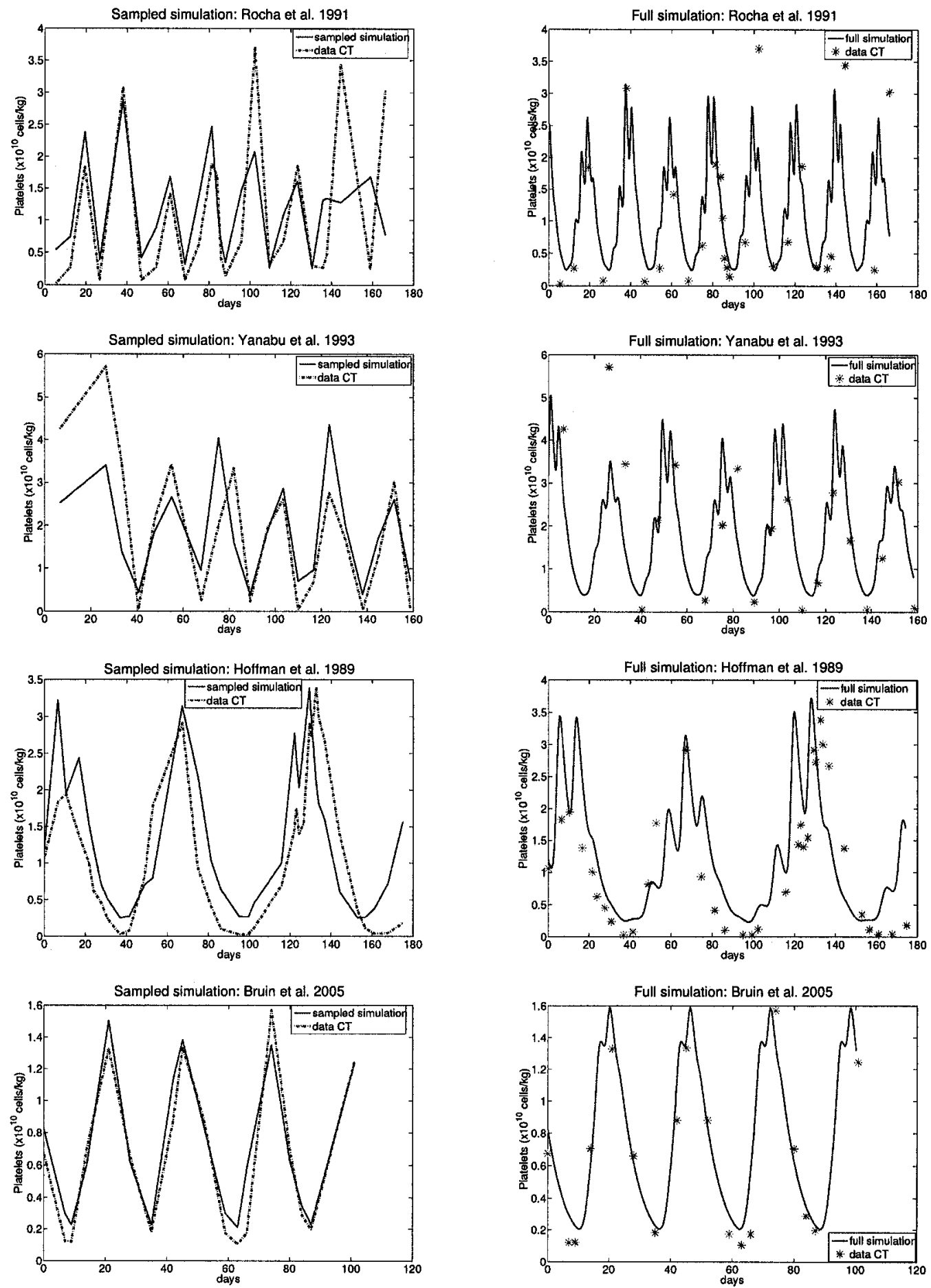

Fig. 5.9 Output of the simulated annealing fitting and the published platelet data. Sampled (left) and full (right) simulation. 

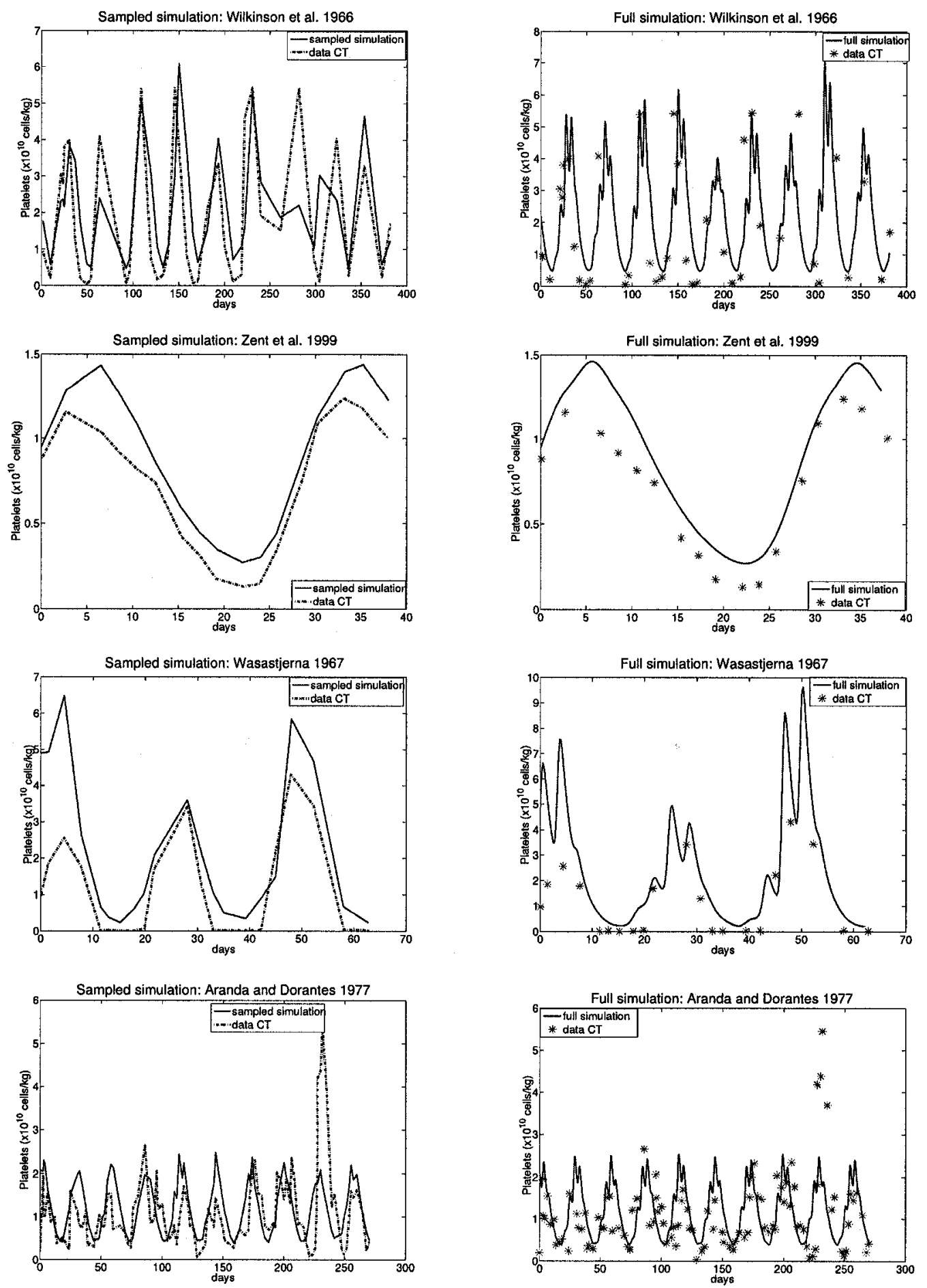

Fig. 5.10 Output of the simulated annealing fitting and the published platelet data. Sampled (left) and full (right) simulation. 

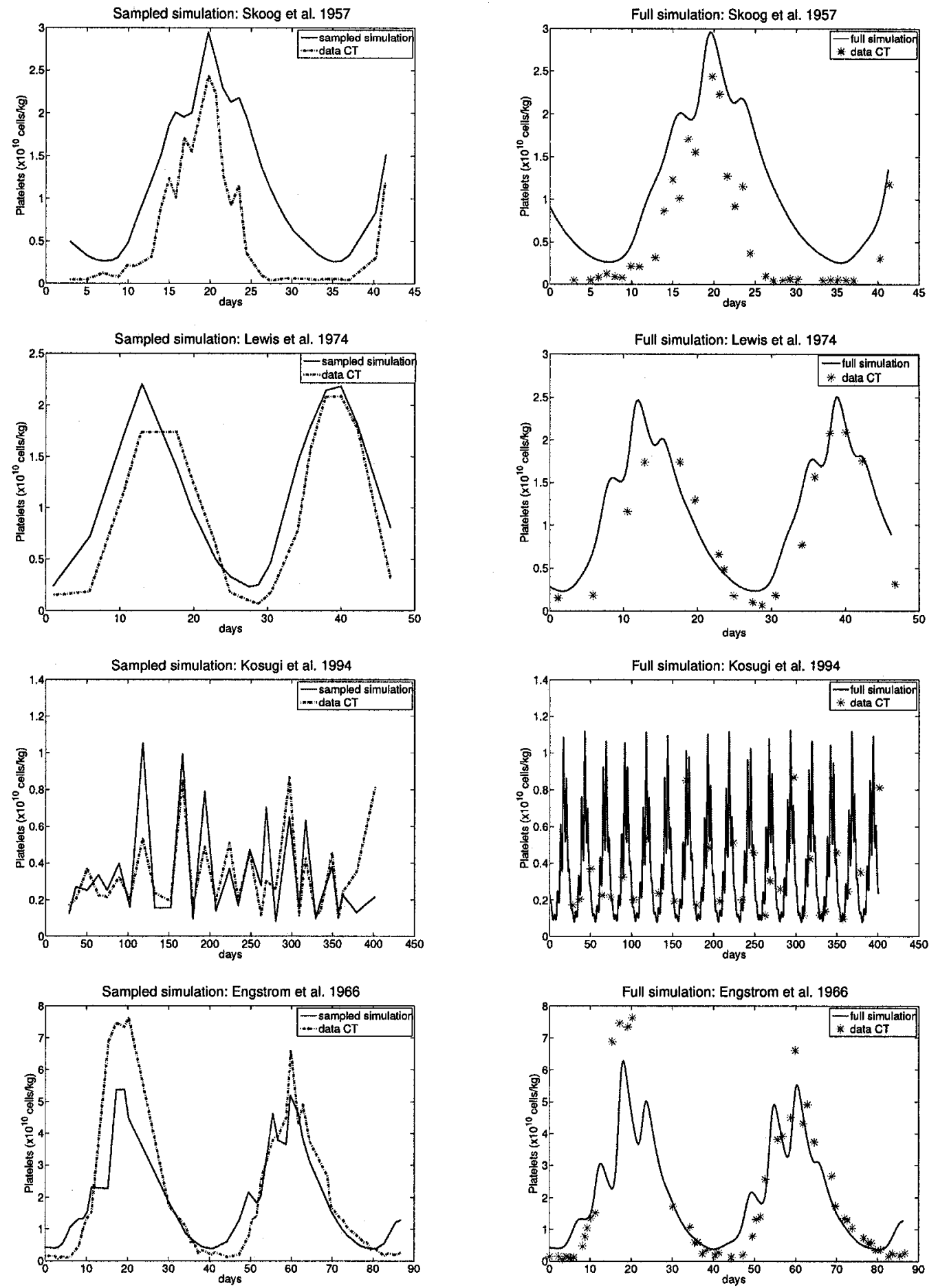

Fig. 5.11 Output of the simulated annealing fitting and the published platelet data. Sampled (left) and full (right) simulation. 


\subsection{Published TPO levels in cyclical thrombocytopenia patients}

Since thrombopoietin is the primary regulator of platelet production, abnormalities of this hormone or its receptor might be responsible for platelet fluctuations. Some research groups $((15),(51),(81),(100))$ measured the platelet counts in CT patients as well as the temporal evolution of TPO concentration. Figure 5.12 shows that for each of the cases mentioned above our model provides a qualitative fitting of the published thrombopoietin levels. This fact validates the accuracy of our model predictions and suggests that the TPO oscillations are secondary to manifestations of some other pathology.
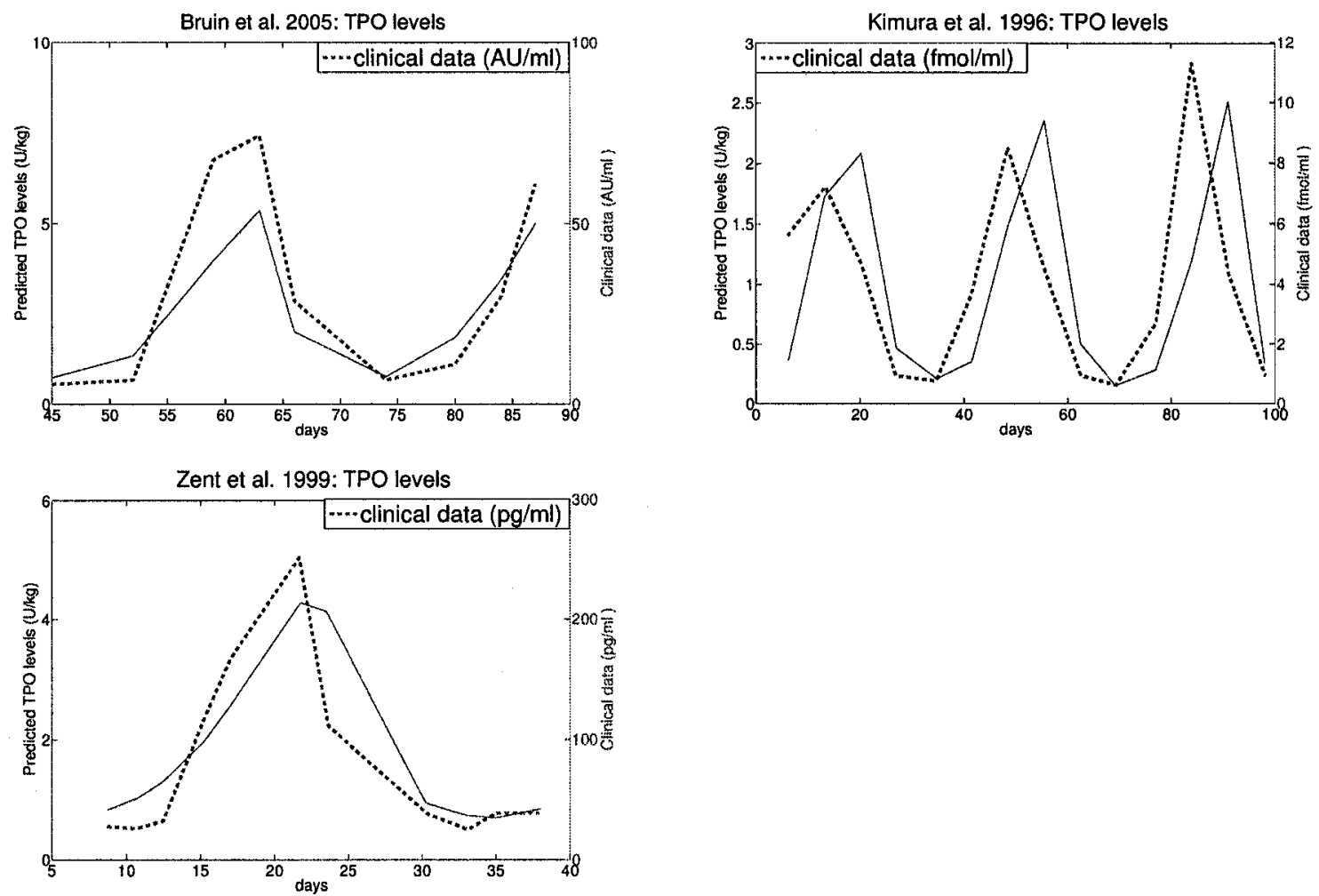

Fig. 5.12 Qualitative comparison of predicted ('-') and published ('- - ') TPO levels. 


\subsection{Hypothesis for the origin of oscillations in cyclical thrombocytopenia}

A variety of modeling studies $[(5 ; 6 ; 8 ; 19 ; 18 ; 20 ; 69 ; 80 ; 83)]$ have associated the onset of oscillations in hematological disease with a Hopf bifurcation induced by the change of one or more physiological parameters. Though the model we have developed here was too complicated for a complete stability analysis we hypothesize that the oscillations seen in $\mathrm{CT}$ and studied here are also due to a Hopf bifurcation.

The mathematical model (3.2.5) successfully duplicated both the qualitative and quantitative features of CT. The platelet fluctuations in amegakaryocytic CT are caused by a cyclic inhibition of megakaryocytopoiesis, accentuated by an increased platelet maturation time and a reduced release of platelets per megakaryocyte. The critical parameter changes required to mimic the corresponding data are a severe decrease in $\mu$, an increase in $\tau_{P M}$ between 2 and 6 times the normal value, and occasionally, a reduced $A_{0}$.

In the case of autoimmune CT, the most significant parameter changes recorded during the successful attempts to fit the amegakaryocytic data are an elevation of $\gamma_{P}$, a decrease of $\mu$, and an increase in $\tau_{P M}$ by a factor of 2 . These results suggest that the onset of oscillations in autoimmune $\mathrm{CT}$ can be explained by an accelerated peripheral destruction of platelets, exacerbated by an increased maturation of megakaryocytes and a slow relative growth rate of megakaryocytes. 

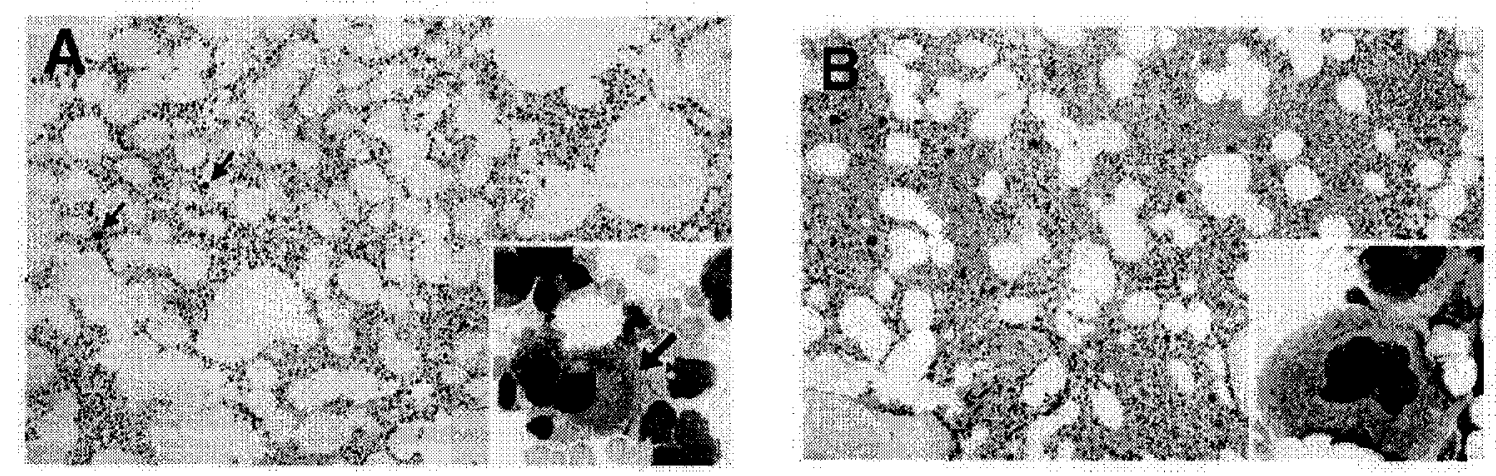

Fig. 5.13 Photomicrographs of the bone marrow biopsy for an amegakaryocytic patient (from Zent et al. (100), Figure 2, p. 454). A. Markedly decreased megakaryocytes (small arrows) while platelet count was decreasing towards nadir. Of the few megakaryocytes seen in the aspirated material (insert) some were atypical micromegakaryocytes (large arrow) with small nuclei and mature cytoplasm. B. Markedly decreased megakaryocytes (small arrows) with normal morphological features (insert) while platelet count was increasing from nadir. 


\section{Chapter 6}

\section{Conclusions}

\subsection{Human TPO levels: megakaryocyte deficiency versus increased platelet destruction}

Over the years, clinical measurements have revealed a negative relationship between the TPO levels and platelet count. This fact led to the hypothesis that the endogenous TPO level is mainly regulated by a consumption process in which TPO binds to the $\mathrm{C}-\mathrm{Mpl}$ receptor and then is removed from circulation. This consumption process is directly dependent upon platelet mass ((32), (33), (60), (89)). However, there are some situations when the thrombopoietin levels are not accurately predicted by the circulating platelet counts. One exception is the case of hematological disorders caused by either megakaryocyte deficiency (like in aplastic anaemia) or increased platelet destruction (as in immune thrombocytopenic purpura) (25). Immune thrombocytopenic purpura (ITP) is characterized by persistent thrombocytopenia caused by circulating anti-platelet autoantibodies which results in platelet destruction by the reticuloendothelial system (55). Aplastic anaemia (AA) is a rare, potentially fatal disease, in which the bone marrow fails to produce sufficient blood cells for circulation. Despite a similar degree of thrombocytopenia in both groups of patients, the levels of TPO in ITP cases are normal or slightly higher, while in AA patients they were markedly elevated ((25), (55), (73)).

Besides increased platelet maturation time, the main causes of cyclical thrombocy- 


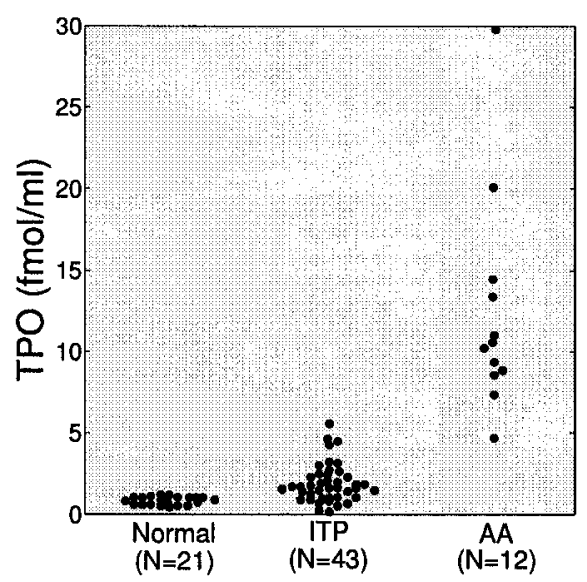

Fig. 6.1 TPO levels in patients with ITP and AA, measured by a sandwich ELISA. Reproduced from Kosugi et al. (55).

topenia are either immune mediated platelet destruction or deficiency of megakaryocytopoiesis. Since our model was able to fit the platelet data, and to duplicate quantitatively the TPO concentration (when data available), it would be of interest to predict and analyze the thrombopoietin levels for all CT cases. Precisely, we are interested to compare the TPO production in autoimmune CT and amegakaryocytic $\mathrm{CT}$, and to determine if the levels of the hormone in the case of increased platelet clearance are as high as would be anticipated by the degree of thrombocytopenia. In all the cases studied TPO concentration mirrors the platelet count. An elevated production corresponds to a decrease in platelet count, and reciprocal. The reader is referred to Appendix B for the predicted TPO response in CT patients. These findings can be explained by the following reasons:

(i) markedly increased platelet turnover rate (22).

(ii) In cyclical thrombocytopenia, beside accelerated peripheral destruction of platelets and inhibition of the megakaryocytopoiesis, the mechanism of platelet fluctuations involves other factors (e.g. periodic variation in the maturity of megakaryocytes, reduced number of platelets released per megakaryocyte).

(iii) Modeling the TPO kinetics we followed the pathway argued by experimental 
data from (32), (33), (60), (89), and we considered the thrombopoietin levels as a function of the total number of circulating platelets.

\subsubsection{TPO kinetics in our model}

The TPO kinetics in our model is based on the previous work of Santillán et al. (83). They assumed that the platelet production is controlled by a negative feedback mechanism mediated by TPO. The concentration of thrombopoietin, denoted T, satisfies the ordinary differential equation:

$$
\frac{d T}{d t}=\underbrace{f(P)}_{\text {production }}-\underbrace{\kappa T}_{\text {destruction }} \quad \text { (equation (12) from Santillán et al. (83)) }
$$

In determining the form of the production function $f(P)$, which is dependent on the total number of circulation platelets $P$, and the normal values of the parameters involved, Santillán et al. (83) used the experimental data for sheep with different degrees of induced thrombocytopenia provided by Kuter (62). A convenient function that captures the features of platelet-TPO relationship is

$$
f(P)=\frac{a}{1+K_{P} P^{r}} \quad \text { (equation (13) from Santillán et al. (83)) }
$$

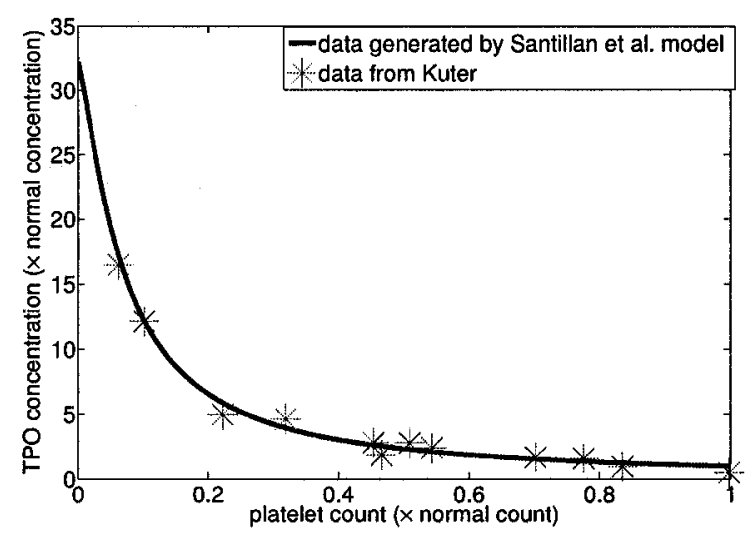

Fig. 6.2 TPO concentration as a function of platelet count. Reproduced from Santillán et al. (83), Figure 2, p. 591. 

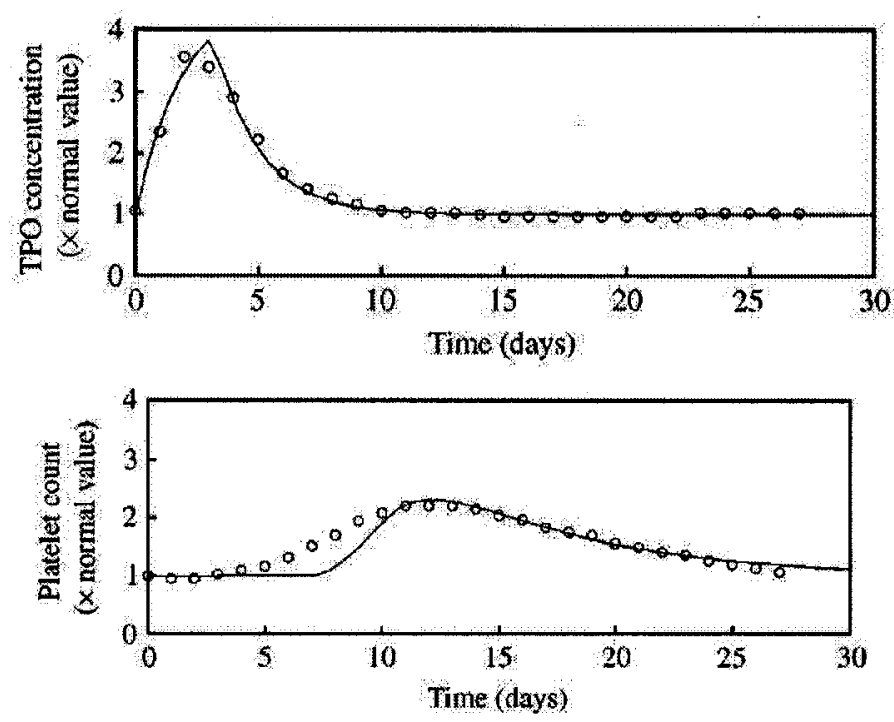

Fig. 6.3 TPO and platelet dynamics after a bolus intramuscular injection of thrombopoietin in healthy humans (experimental data from Harker et al. (42)). Reproduced from Santillán et al. (83), Figure 8, p. 596.

Consider that TPO concentration is in dynamic equilibrium with the number of circulating platelets. Then

$$
\frac{d T}{d t}=0 \quad \text { and } \quad T=\frac{a}{\kappa} \times \frac{1}{1+K_{P} P^{r}} .
$$

Denote by $\widetilde{T}$ and $\widetilde{P}$ the quasi-steady state established by the experimental protocol. Therefore the normalized steady state values $\frac{\widetilde{T}}{T_{*}}$ and $\frac{\widetilde{P}}{P_{*}}$ satisfy the equation

$$
\frac{\widetilde{T}}{T_{*}}=\frac{a}{\kappa T_{*}} \times \frac{1}{1+\left(K_{P} P_{*}\right)^{r}\left(\frac{\widetilde{P}}{P_{*}}\right)^{r}} .
$$

Fitting the above equation to the Kuter's data (Figure 6.2) one finds the normal values of all parameters:

$$
\frac{a}{\kappa}=32.18 T_{*}, \quad K=\frac{31.18}{P_{*}^{r}}, \quad r=1.29 .
$$


Moreover, the model for platelet regulation production from (83) reproduces very well the dynamics of TPO concentration and platelet counts after a bolus intramuscular injection of thrombopoietin in healthy humans (see Figure 6.3).

\subsubsection{Are the TPO levels exclusively dependent on the platelet number?}

Since the mechanism of the regulation of the thrombopoietin production is not completely understood, it is difficult to explain why the patterns of reactive TPO production against thrombocytosis in megakaryocyte deficiency and increased platelet destruction are different. Recently, some research groups formulated a hypothesis which is presented within the following context.

Although it has not been clearly demonstrated up to now, it has been suggested that the TPO levels are not exclusively dependent on the platelet number, and the megakaryocyte mass play an important role in regulating the serum thrombopoietin levels in human subjects (87). To clarify this controversial point, Nagasawa et al (74) analyzed the relationship between the serum TPO level, platelet count, megakaryocyte and CFU-Meg number in a female patient whose megakaryopoiesis switched from ITP to amegakaryocytic phase during chemotherapy for lymphoma. They found that the TPO level was not suppressed in response to the platelet count, but the changes in the hormone were the mirror image of the variations in the megakaryocyte mass. Mukai et al (73) examined the thrombopoietin concentration, platelet and megakaryocyte counts in a patient with amegakaryocytic thrombocytopenia during steroid treatment. Their data showed that the decrease in the TPO concentration preceded the increase in platelet counts. Similarly, Oh et al. (76) measured the serum TPO levels in a woman diagnosed with cyclical thrombocytopenia. TPO decreased slightly before platelets began to rise. Engel et al. (26) examined three patients under chemotherapy intensified treatment. Again, the thrombopoietin response preceded the platelet response by about one day. Unusual in their measurements was the quantitative change in platelet and TPO dynamics over successive cycles. More precisely, while the platelet nadirs decreased with each cycle, the TPO concentration increased concomitantly. A possible explanation for the inadequate increase in the thrombopoietin response over cycles is related to the loss in the number 
of receptors on platelets and megakaryocytes, loss due to a cumulative damage of thrombopoiesis. Zent et al. (100) examined a male patient with amegakaryocytic thrombocytopenic purpura, and determined the TPO response to intrinsic changes in platelet and megakaryocyte mass in the absence of cytotoxic drugs, radiotherapy or antiplatelet antibodies. Thrombopoietin concentration rapidly decreased concomitant with the increase in the megakaryocyte mass, and well before the next rise in platelet count. Based on these laboratory studies, some research groups ((26), (55), (74), (87), (100)) argue that the megakaryocytes have a great importance in the regulation of thrombopoietin metabolism, and consider the observed time lag between the TPO and platelet response as a consequence of this fact (26). Kosugi et al. (55) adopt this theory, and believe that it might explain the concentration of TPO in ITP versus AA. In ITP megakaryocytes are abundant in bone marrow and a great number of platelets enter the circulation. In contrast, in AA there is an insufficient number of megakaryocytes, and only few platelets are released into circulation. Therefore, one
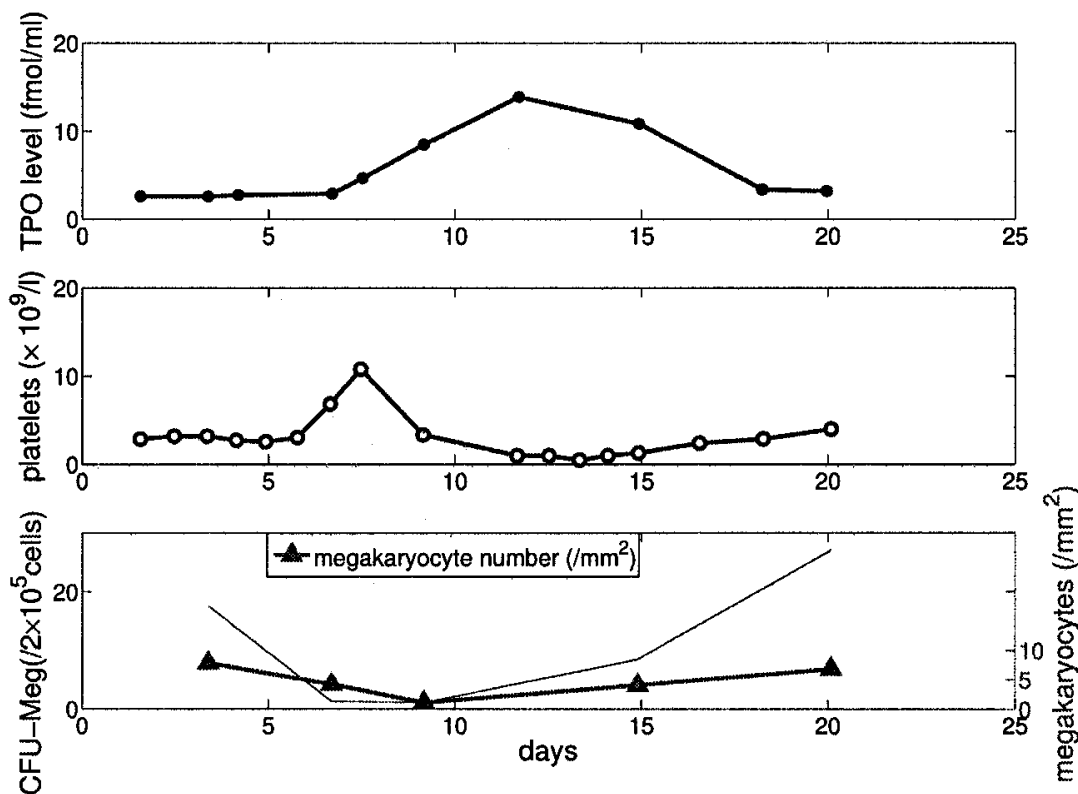

Fig. 6.4 TPO response related to the megakaryocyte number rather than platelet counts. Reproduced from Nagasawa et al. (74), Fig 1., p. 243. 
should expect higher hormone levels in patients with aplastic anaemia.

The relative role of circulating platelets and bone marrow megakaryocytes in TPO regulation is uncertain. The understanding of the molecular basis of thrombopoiesis is under intense research and the years to come will clarify the questions unanswered up to now.

\subsection{A short review}

The goal of this research project was to explain through computer simulations the mechanism of the rhythmic fluctuations in the platelet count observed in cyclical thrombocytopenia. After a presentation of the physiological framework and the clinical features of CT (Chapter 1), we analyzed the platelet data published in the literature. Since the data was unevenly sampled, it was possible to extract information about the oscillatory components through the Lomb-Scargle periodogram (Chapter 2). To provide a better understanding of CT, we employed a mathematical model of hematopoiesis. The model, introduced in Chapter 3, had four compartments and was comprised by four delay differential equations. All compartments, with one exception, were based on the previous modeling work of Colijn and Mackey (18). The mathematical analysis performed in Chapter 4 showed that the model has a unique positive steady state which corresponds to the normal levels in healthy individuals (Section 4.2). The linear stability analysis around the positive equilibrium point revealed the model dynamics in a neighborhood of the fixed point, and identified regions in the parameter space where the model displays unstability (Section 4.3). Chapter 5 was entirely devoted to the understanding of the mechanisms underlying the platelet fluctuations in CT. Our model successfully duplicated the platelet counts of CT patients and provided a qualitative fitting of the thrombopoietin levels (when data available). The fitting followed a simulated annealing algorithm with Metropolis acceptance rule and a constant thermodynamic speed schedule. In the case of some hematological disorders caused by either megakaryocyte deficiency or increased platelet destruction, the thrombopoietin levels are not accurately predicted by the circulating platelet counts. Since the understanding of the molecular basis of thrombopoiesis is not completely elucidated, we compared the TPO production in 
autoimmune CT and amegakaryocytic CT using the predictions of our model (Section 6.1). The biological interpretation of the results, the comparison with the clinical findings, and the conclusions drawn from the previous modeling effort are synthesized in the last chapter.

\subsection{Discussion and future directions}

The pathogenesis of CT is not clearly understood and remains speculative. Clinical attempts undertaken over the years to explain the aetiology of platelet fluctuations led to different conclusions. Generally, the onset of oscillations was explained in terms of immune-mediated platelet destructions or periodic failure of platelet production. To our knowledge, only two modeling works have been directed to the mathematical investigation of CT. Von Schulthness and Gessner (92) suggested that, in the case of their asymptomatic patients, the platelet control was biased close to a stability boundary (91), (92). Santillán et al. (83) formulated an age-structured model for the regulation of the platelet production, and reproduced the features of autoimmune CT by increasing the death rate of circulating platelets between twice and ten times the normal value. Moreover, they believed that autoimmune and amegakaryocytic CT have a different dynamical origin.

Our mathematical model (3.2.5) successfully duplicated the platelet counts of CT patients and provided a qualitative fitting of the thrombopoietin levels (when data available). The results of our investigation are in agreement with the clinical findings, explain the experimental observations, partially support the conclusions drawn from the previous modeling effort, and uncover new factors responsible for the fluctuating pattern in platelets. Based on the model simulations, we would suggest that autoimmune and amegakaryocytic CT share common features and display important differences. The variation in the megakaryocyte maturity, the relative growth rate of megakaryocytes, as well as the random destruction of platelets are involved in both varieties of $\mathrm{CT}$, but up to a different extent. Comparable to amegakaryocytic $\mathrm{CT}$, in autoimmune CT the rate of platelet clearance is higher, the megakaryocytes spend less time in the bone marrow, and their relative growth rate is closer to the normal value. Additionally, the model was particularly sensitive to changes in $A_{0}$ for some 
amegakaryocytic patients, implying a diminished platelet release per megakaryocyte. Sometimes it was hard to clinically differentiate between autoimmune and amegakaryocytic CT. In few reported cases $((34),(51),(81))$ the deficiency of platelets due to a fluctuating number of megakaryocytes was exacerbated by an increased platelet clearance caused by the presence of raised levels of platelet autoantibodies. Since in thrombocytopenic cases with autoimmune causes the TPO levels are not as high as it would be expected, it has been suggested that a measurement of the cytokine's concentration might help for a better diagnosis (74).

Based on the laboratory results, it was postulated that in autoimmune CT the platelet fluctuations are secondary to an elevated platelet destruction. The numerical experiments performed with our model show that increasing only $\gamma_{P}$ is not sufficient to reproduce the platelet data of autoimmune patients.

Although this thesis ends with the formulation of a possible mechanism underlying the platelet rhythmic fluctuations in cyclical thrombocytopenia, our work points to new directions for further research. Once we have identified the crucial parameters in duplicating the features of CT, we are ready to explore more systematic the model dynamics. One interesting idea would be to track the Hopf bifurcation point(s) believed to generate oscillations in the platelet compartment and to follow the branches of periodic solutions. Another topic would be related to the model improvement. Since it was speculated that the failure of megakaryocytopoiesis in amegakaryocytic CT appears at the stages of CFU-Meg ((21), (73)), a mathematical model which counts for the multiple levels of megakaryocyte transition from lineage committed cells to release of platelets could explore this possibility. 


\section{Appendix A}

\section{Detailed calculations for the steady state(s) of the model (3.2.5)}

The computations from Section 4.2 show that the steady state values are defined implicitly by the solutions of the following nonlinear system:

$$
\left\{\begin{aligned}
\beta\left(Q_{*}\right) Q_{*} & =\left\{-\left(\kappa_{N}\left(N_{*}\right)+\kappa_{P}+\kappa_{R}\left(R_{*}\right)\right)+2 e^{-\gamma_{S} \tau_{S}} \beta\left(Q_{*}\right)\right\} Q_{*} \\
\gamma_{N} N_{*} & =A_{N} \kappa_{N}\left(N_{*}\right) Q_{*} \\
\gamma_{R} R_{*} & =A_{R} \kappa_{R}\left(R_{*}\right)\left(1-e^{-\gamma_{R} \tau_{R S}}\right) Q_{*} \\
\gamma_{P} P_{*} & =\bar{A}_{P}^{*} \kappa_{P}\left(1-e^{-\gamma_{P} \tau_{P S}}\right) Q_{*}
\end{aligned}\right.
$$

Rearranging conveniently the terms in the $1^{\text {st }}$ equation we obtain:

$$
\beta\left(Q_{*}\right) Q_{*}\left(1-2 e^{-\gamma_{S} \tau_{S}}\right)=\left\{-\left(\kappa_{N}\left(N_{*}\right)+\kappa_{P}+\kappa_{R}\left(R_{*}\right)\right\} Q_{*}\right.
$$

Since

$$
\beta\left(Q_{*}\right)=k_{0} \frac{\theta_{2}{ }^{s}}{\theta_{2}{ }^{s}+Q_{*}^{s}}, \quad \kappa_{N}(N)=f_{0} \frac{\theta_{1}{ }^{n}}{\theta_{1}^{n}+N_{*}^{n}} \quad \text { and } \quad \kappa_{R}\left(R_{*}\right)=\frac{\overline{\kappa_{r}}}{1+K_{r} R_{*}^{m}}
$$


(A.0.2) is equivalent to

$$
\begin{aligned}
\frac{Q_{*}}{\theta_{2}^{s}+Q_{*}^{s}}= & \underbrace{\frac{f_{0} \theta_{1}^{n}}{k_{0} \theta_{2}^{s}\left(2 e^{-\gamma_{S} \tau_{S}}-1\right)}}_{a_{1}} \frac{Q_{*}}{\theta_{1}^{n}+N_{*}^{n}}+\underbrace{\frac{\bar{\kappa}_{r}}{k_{0} \theta_{2}^{s}\left(2 e^{-\gamma_{S} \tau_{S}}-1\right)}}_{a_{2}} Q_{*}+ \\
+ & \underbrace{\frac{\kappa_{P}}{k_{0} \theta_{2}^{s}\left(2 e^{-\gamma_{S} \tau_{S}}-1\right)}}_{a_{3}} \frac{Q_{*}}{1+K_{r} R_{*}^{m}} .
\end{aligned}
$$

Writing explicitly the differentiation rates $\kappa_{N}$ and $\kappa_{R}$, and separating $Q_{*}$ in the $2^{\text {nd }}$ and the $3^{\text {rd }}$ equations of the system (A.0.1), we obtain:

$$
\begin{aligned}
& Q_{*}=\underbrace{\frac{\gamma_{N}}{f_{0} A_{N} \theta_{1}^{n}} N_{*}\left(\theta_{1}^{n}+N_{*}^{n}\right) \text { and }}_{a_{4}} \\
& Q_{*}=\underbrace{\frac{\gamma_{R}}{A_{R} \overline{k_{r}}\left(1-e^{-\gamma_{R} T_{R S}}\right)}}_{a_{5}} R_{*}\left(1+K_{r} R_{*}^{m}\right) .
\end{aligned}
$$

At steady state the average number of platelets released per megakaryocyte is

$$
{\overline{A_{P}}}^{*}=A_{0} e^{\mu \tau_{P M} T_{*}}
$$

and the normal TPO concentration is given by

$$
T_{*}=\frac{T_{\max }}{1+K_{p} P_{*}^{r}}
$$

Substituting these quantities in the $4^{\text {th }}$ equation of (A.0.1) it follows that:

$$
Q_{*}=\underbrace{\frac{\gamma_{P}}{A_{0} K_{P}\left(1-e^{\left.-\gamma_{P} \tau_{P S}\right)}\right.}}_{a_{6}} P_{*} \exp \left(-\frac{\mu \tau_{P M} T_{\max }}{1+K_{P} P_{*}^{r}}\right) .
$$


Let $a_{7}:=\mu \tau_{P M} T_{m a x}$. The above computations show that the nonlinear system (A.0.1) is equivalent to:

$$
\begin{aligned}
\frac{Q_{*}}{\theta_{2}^{s}+Q_{*}^{s}} & =a_{1} \frac{Q_{*}}{\theta_{1}^{n}+N_{*}^{n}}+a_{2} Q_{*}+a_{3} \frac{Q_{*}}{1+K_{r} R_{*}^{m}} \\
Q_{*} & =a_{4} N_{*}\left(\theta_{1}^{n}+N_{*}^{n}\right) \\
Q_{*} & =a_{5} R_{*}\left(1+K_{r} R_{*}^{m}\right) \\
Q_{*} & =a_{6} P_{*} \exp \left(-\frac{a_{7}}{1+K_{P} P_{*}^{r}}\right)
\end{aligned}
$$

Since

$$
\frac{Q_{*}}{\theta_{1}^{n}+N_{*}^{n}}=a_{4} N_{*} \quad\left(\text { from A.0.4) } \quad \text { and } \quad \frac{Q_{*}}{1+K_{r} R_{*}^{m}}=a_{5} R_{*} \quad\right. \text { (from A.0.5) }
$$

equation (A.0.3) becomes

$$
Q_{*} \frac{1}{\theta_{2}^{s}+Q_{*}^{s}}=\underbrace{a_{1} a_{4}}_{a_{8}} N_{*}+a_{2} Q_{*}+\underbrace{a_{3} a_{5}}_{a_{9}} R_{*}
$$

which yields

$$
a_{8} N_{*}+a_{9} R_{*}=Q_{*}\left(\frac{1}{\theta_{2}^{s}+Q_{*}^{s}}-a_{2}\right)
$$

In conclusion, the investigation of the steady states of the model (3.2.5) is reduced to the study of the nonlinear system:

$$
\left\{\begin{aligned}
a_{4} N_{*}\left(\theta_{1}^{n}+N_{*}^{n}\right) & =Q_{*} \\
a_{5} R_{*}\left(1+K_{r} R_{*}^{m}\right) & =Q_{*} \\
a_{8} N_{*}+a_{9} R_{*} & =Q_{*}\left(\frac{1}{\theta_{2}^{s}+Q_{*}^{s}}-a_{2}\right) \\
a_{6} P_{*} \exp \left(-\frac{a_{7}}{1+K_{P} P_{*}^{r}}\right) & =Q_{*}
\end{aligned}\right.
$$




\section{Appendix B}

\section{Predicted thrombopoietin levels in CT patients}
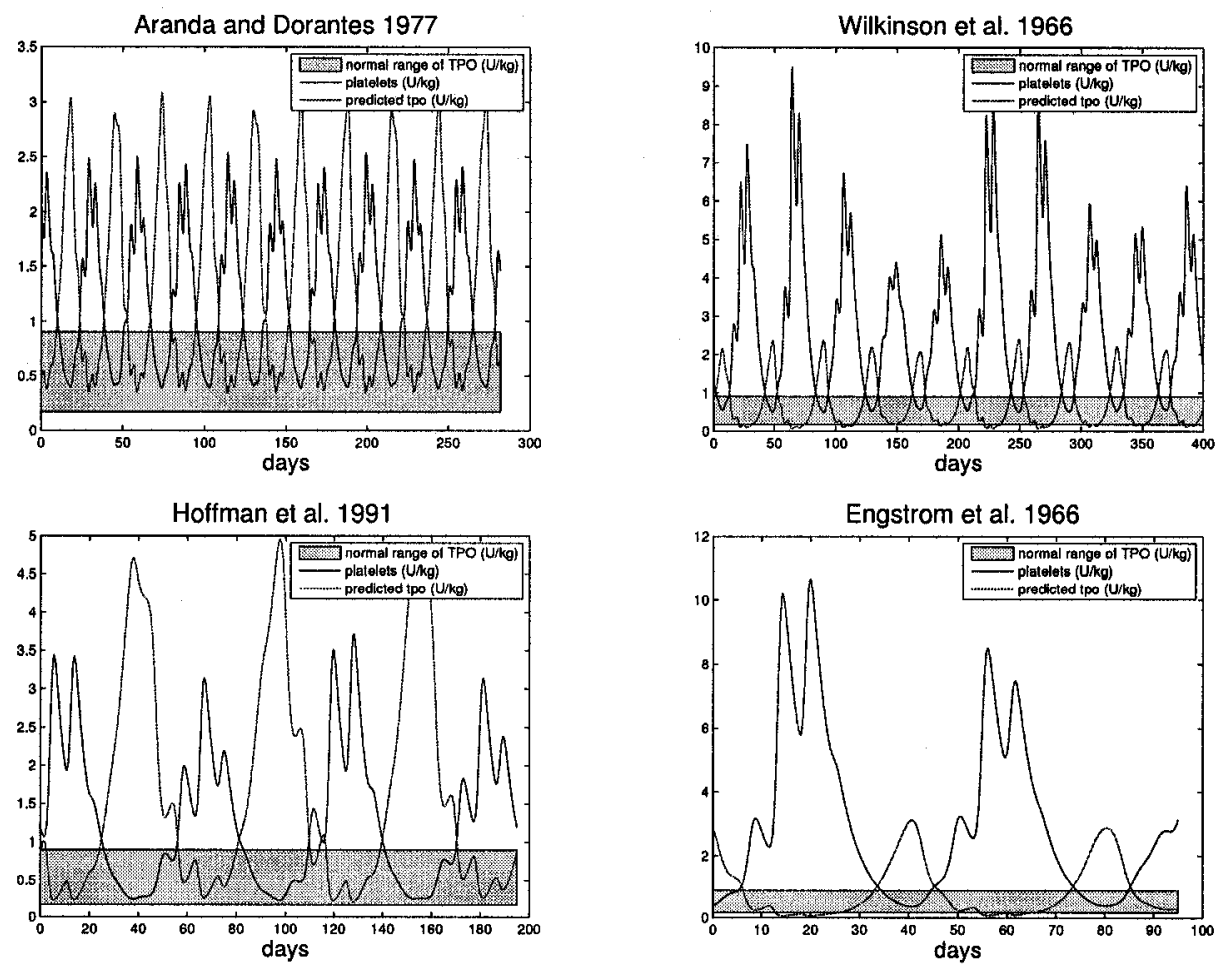

Fig. B.1 Prediction of the thrombopoietin levels for amegakaryocytic patients. 

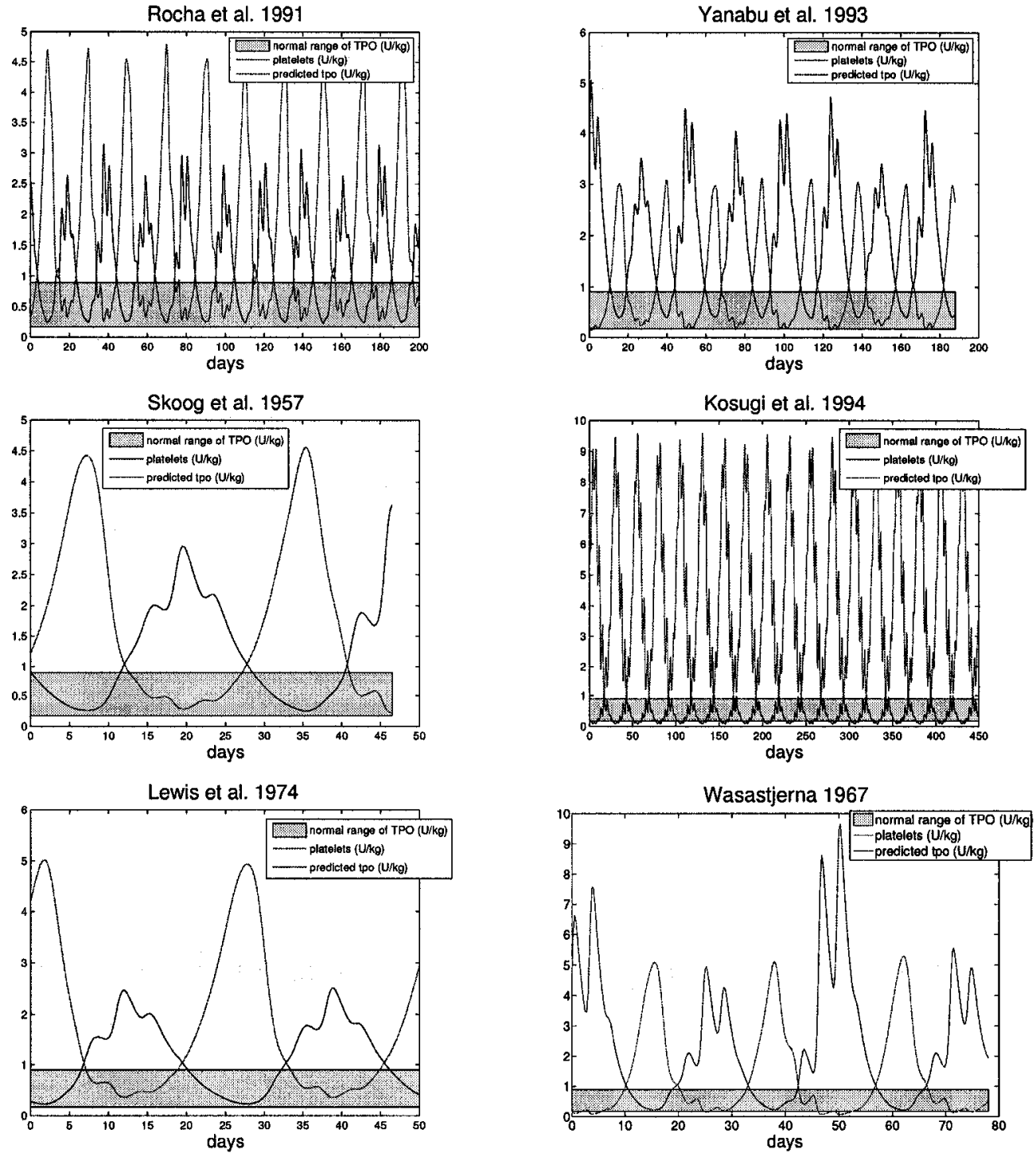

Fig. B.2 Prediction of the thrombopoietin levels for patients diagnosed with C-TPO (Levis et al.), amegakaryocytic CT (Wasastjerna), and autoimmune CT (all the others). 


\section{Appendix C}

\section{XPP code}

The mathematical model (3.2.5) is specified by four integral delay differential equations. Since the function 'int' incorporated in the software XPP performs a slow computation of the integrals, we chose to numerically approximate the average TPO concentration

$$
\bar{T}(t)=\frac{1}{\tau_{P M}} \int_{t-\tau_{P M}}^{t} T\left(t^{\prime}\right) d t^{\prime}
$$

using Newton-Cotes formulas. Below is an example which uses an 11-point NewtonCotes formula.

\# Steady state parameter values par $A N=3000, A R=5.6276$, kappaP=0.028, $A 0=0.1, \operatorname{miu}=1.7836$

par gammaS=0.07, gammaN=2.4, gammaR=0.001, gammaP=0.15

par $0 \max =13.77, K P=11.68, r 3=1.29, f 0=0.1$, kappaRbar=1.1738, r2 $=1$

par tauS $=2.8$, tauNM=3.5, tauRM $=6, \operatorname{tauRS}=120, \operatorname{tauPM}=7$, tauPS $=9.5$

par tauRsum $=126$

par $\mathrm{k} 0=8$, theta $2=0.095, \mathrm{~s}=2$, theta1 $=0.36, \mathrm{KR}=0.0382, \mathrm{r} 1=6.96$

\# Initial conditions

$Q(0)=1.1$

$\mathrm{N}(0)=6.9$

$R(0)=3.500$ 
$P(0)=2.143$

\# Equations

$\mathrm{dP} / \mathrm{dt}=-$ gammaP $* \mathrm{P}+\mathrm{A} 0 * \exp \left(\operatorname{miu} * \operatorname{tauPM} * 0 \max *\left(0.0268 /\left(1+\mathrm{KP} * \mathrm{P}^{\wedge} \mathrm{r} 3\right)+\right.\right.$

$0.0268 /(1+K P * \operatorname{delay}(\mathrm{P}$, tauPM $) \wedge \mathrm{r} 3)+0.1775 /(1+\mathrm{KP} *$

$\operatorname{delay}(P, 0.1 * \operatorname{tau} P M) \wedge r 3)+0.1775 /(1+K P * \operatorname{delay}(P, 0.9 * \operatorname{tau} P M) \wedge r 3)-$

$0.081 /(1+\mathrm{KP} * \operatorname{delay}(\mathrm{P}, 0.8 * \mathrm{tauPM}) ` \mathrm{r} 3)-0.081 /(1+\mathrm{KP} *$

$\operatorname{delay}(P, 0.2 * \operatorname{tauPM}) \wedge \mathrm{r} 3)+0.4549 /(1+\mathrm{KP} * \operatorname{delay}(\mathrm{P}, 0.7 * \operatorname{tauPM}) ` \mathrm{r} 3)+$

$0.4549 /\left(1+K P * \operatorname{del}\right.$ ay $\left.(\mathrm{P}, 0.3 * \mathrm{tauPM})^{\wedge} \mathrm{r} 3\right)-0.4351 /(1+\mathrm{KP} *$

$\left.\operatorname{delay}(P, 0.6 * \operatorname{tau} P M)^{\wedge} r 3\right)-0.4351 /(1+K P * \operatorname{delay}(P, 0.4 * \operatorname{tau} P M) ` r 3)+$

$\left.\left.0.7137 /\left(1+K P * \operatorname{delay}(P, 0.5 * \text { tauPM })^{`} r 3\right)\right)\right) * k a p p a P *$

(delay $(Q$, tauPM) $-\exp (-\operatorname{gammaP} * \operatorname{tau} P S) * \operatorname{delay}(\mathrm{Q}$, tauPM+tauPS$))$

$\mathrm{dQ} / \mathrm{dt}=-(\operatorname{kappaN}(\mathrm{N})+\operatorname{kappaR}(\mathrm{R})+\operatorname{kappaP}+\operatorname{beta}(\mathrm{Q})) * \mathrm{Q}+2 * \exp (-\operatorname{gammaS} * \operatorname{tau} S) *$

$\operatorname{delay}(Q, \operatorname{tau} S) * \operatorname{beta}(\operatorname{delay}(Q, \operatorname{tau} S))$

$\mathrm{dN} / \mathrm{dt}=-\operatorname{gammaN} * \mathrm{~N}+\mathrm{AN} * \operatorname{kappaN}(\operatorname{delay}(\mathrm{N}, \operatorname{tau} M)) * \operatorname{delay}(\mathrm{Q}, \operatorname{tauNM})$

$\mathrm{dR} / \mathrm{dt}=-\operatorname{gammaR} * \mathrm{R}+\mathrm{AR} *(\operatorname{kappaR}(\operatorname{delay}(\mathrm{R}, \operatorname{tauRM})) * \operatorname{delay}(\mathrm{Q}, \operatorname{tauRM})-$

$\exp (-\operatorname{gammaR} * \operatorname{tauRS}) * \operatorname{kappaR}(\operatorname{delay}(\mathrm{R}, \mathrm{tauRsum})) * \operatorname{del}$ ay $(\mathrm{Q}, \mathrm{tauRsum}))$

\# The negative feedback functions take the form of Hill functions

$\operatorname{beta}(Q)=k 0 * \operatorname{theta} 2 \wedge s /($ theta2^s+Q^s)

$\operatorname{kappaN}(N)=f 0 * \operatorname{theta1}^{\wedge} \mathrm{r} 2 /\left(\right.$ theta1 $\left.1^{\wedge}\{\mathrm{r} 2\}+\mathrm{N}^{\wedge}\{\mathrm{r} 2\}\right)$

$\operatorname{kappaR}(\mathrm{R})=\operatorname{kappaRbar} /\left(1+\mathrm{KR} * \mathrm{R}^{\wedge}\{\mathrm{r} 1\}\right)$

\#

(C) delay $=200$, maxstor $=10000$, noutput $=5$, bounds $=10 e+10$

Q $x$ low $=200, x h i=500$, total $=500, y l o w=0, y h i=11$

done 


\section{References}

[1] M. Adimy, F. Crauste, Modelling and Asymptotic Stability of a Growth FactorDependent Stem Cells Dynamics Model with Distributed Delays, To appear in Discrete and Continuous Dynamical Systems, Series B (2006).

[2] J. W. N. Akkerman, Thrombopoietin and Platelet Function, Seminars in Musculoskeletal Radiology (2006), 32, 295-304.

[3] E. Aranda, S. Dorantes, Garcia's disease: Cyclic thrombocytopenic purpura in a child and abnormal platelet counts in his family, Scand. J. Haematol. (1977), $18,39-46$.

[4] C. L. Balduini, C. C. Stella, V. Rosti, G. Bertolino, P. Noris, E. Ascari, Acquired cyclic thrombocytopenia-thrombocytosis with periodic defect of platelet function, Brit. J. Haematol (1993), 85, 718-722.

[5] J. Bélair, M. C. Mackey, A model for the regulation of mammalian platelet, Ann. N.Y. Acad. Sci. (1987), 504, 280-282.

[6] J. Bélair, M. C. Mackey, J. M. Mahaffy, Age-structured and two-delay models for erythropoiesis, Math. Biosci. (1995), 128, 317-346.

[7] R. Bellman, K. L. Cooke, Modern elementary differential equations, Edition 2d ed., Published Reading, Mass., Addison-Wesley Pub. Co., 1971.

[8] S. Bernard, J. Bélair, M. C. Mackey, Oscillations in Cyclical Neutropenia: new evidence based on mathematical modeling, J. Theor. Biol. (2003), 223, 283-298.

[9] S. Bernard, J. Bélair, M. C. Mackey, Bifurcations in a white blood cell production model, Comptes Rendus Biologiques (2004), 327, 201-210.

[10] E. Berreta, Y. Kuang, Geometric stability switch criteria in delay differential systems with delay dependent parameters, SIAM J. Math. Anal. (2002), 33, 5, 1144-1165. 
[11] A. Beuter, L. Glass, M. C. Mackey, M. S. Titcombe, Nonlinear Dynamics in Physiology and Medicine, Springer-Verlag, New York, 2003.

[12] E. Beutler, M. A. Lichtman, B. S. Coller, T. J. Kipps, Williams Hematology, McGraw-Hill, New-York, 1995.

[13] I. Branehog, J. Kutti, B. Ridell, B. Swolin, A. Weinfeld, The relation of thrombokinetics to bone marrow megakaryocytes in ideopatic thrombocytopenic purpura (ITP), Blood (1975), 45, 552-562.

[14] O. Brey, E. P. R. Garner, D. Wells, Cyclical Thrombocytopenia Associated with the Multiple Autoantibodies, Br. Med. J. (1969), 3, 397-398.

[15] M. Bruin, M. R. Tijssen, M. Bierings, M. de Haas, Juvenile Cyclic Amegaryocytoc Thrombocytopenia: A Novel Entity, J. Pediatr. Hematol. Oncol. (2005), 27, 3, 148-152.

[16] V. Cerný, A thermodynamical approach to the travelling salesman problem: an efficient simulation algorithm, Journal of Optimization Theory and Applications (1985), 45, 41-51.

[17] T. Cohen, D. P. Cooney, Cyclic thrombocytopenia. Case report and review of literature, Scand. J. Haematol. (1974), 12, 9-17.

[18] C. Colijn, M. C. Mackey, A mathematical model of hematopoiesis: Periodic chronic myelogenous leukemia, part I, J. Theor. Biol. (2005), 237, 117-132.

[19] C. Colijn, M. C. Mackey, A mathematical model of hematopoiesis: Cyclical neutropenia, part II, J. Theor. Biol. (2005), 237, 133-146.

[20] C. Colijn, M. C. Mackey, Bifurcation and bistability in a model of hematopoietic regulation, SIAM J. App. Dynam. Sys. (2005, submitted).

[21] K. Dan, K. Inokuchi, E. An, et al., Cell-mediated cyclic thrombocytopenia treated with azathioprine, Brit. J. Haematol. (1991), 77, 365-370.

[22] V. R. Deutsch, A. Tomer, Megakaryocyte development and platelet production, Brit. J. Haematol. (2006), 134, 453-466.

[23] O. Dickerman, S. A. van Gils, S. M. Verduyn Lunel, H.-O. Walther Delay Equations, Functional-, Complex-, and Nonlinear Analysis, Springer-Verlag, Applied mathematical sciences, vol. 110, 1995.

[24] L. E. El'sgol'ts, S. B. Norkin, Introduction to the theory and application of differential delay equations with deviating argument, Academic Press, 1973. 
[25] R. V. Emmons, D. M. Reid, R. L. Cohen, G. Meng, N. S. Young, C. E. Dunbar, N. R. Shulman, Human thrombopoietin levels are high when thrombocytopenia is due to megakaryocyte deficiency and low when due to increased platelet destruction, Blood (1996), 87, 4068-4071.

[26] C. Engel, M. Loeffler, H. Franke, S. Schmitz, Endogenous thrombopoietin serum levels during multicycle chemotherapy, Brit. J. Haematol. (1999) 105, 832-838.

[27] K. Engelborghs, T. Luzyanina, Numerical bifurcation analysis of delay differential equations using ddebiftool, ACM Trans, Math., 2002.

[28] K. Engelborghs, T. Luzyanina, G. Samaey, DDEBIFTOOL v. 2.00: a Matlab package for bifurcation analysis of delay differential equations, Technical raport, Department of Computer Science, K. U. Leuven, Leuven, Belgium, 2001.

[29] K. Engstrom, A. Lundquist, N. Soderstrom, Periodic thrombocytopenia or tidal platelet dysgenesis in a man, Scand. J. Haemat. (1966), 3, 290-292.

[30] B. Ermentrout, Simulating, Analysing, and Animating Dynamical Systems: A Guide to Xppaut for Researchers and Students (Software, Environments, Tools) 1st Edition, SIAM, Philadelphia, PA, 2001.

[31] B. Ermentrout, XPPAUT version 5.4 software, http://www.math.pitt.edu/ bard/xpp/xpp.html 2001.

[32] P. J. Fielder, A. L. Gurney, E. Stefanich, M. Marian, M. W. Moore, K. CarverMoore, F. J. de Sauvage, Regulation of thrombopoietin levels by c-mpl-mediated binding to platelets, Blood (1996), 87, 2154-2161.

[33] P. J. Fielder, P. Hass, M. Nagel, E. Stefanich, R. Widmer, G. L. Bennet, G. A. Keller, F. J. de Sauvage, D. Eaton, Human platelets as a model for the binding and degradation of thrombopoietin, Blood (1997), 89, 2782-2788.

[34] P. F. Fogarty, M. Stetler-Stevenson, A. Pereira, C. E. Dunbar, Large Granular Lymphocytic Proliferation-Associated Cyclic Thrombocytopenia, American Journal of Hematology (2005), 79, 334-336.

[35] P. Fortin, M. C. Mackey, Periodic chronic myelogenous leukemia: Spectral analysis of blood cell counts and aetiological implications, Brit. J. Haematol. (1999), 104, 336-345.

[36] W. Füreder, G. Mitterbauer, R. Thalhammer, K. Geissler, S. Panzer, M. Krebs, I. Simonitsch-Klupp, W. R. Sperr, K. Lechner, P. A. Kyrle, Clonal T cell-mediated cyclic thrombocytopenia, Brit. J. Haematol. (2002), 119, 1059-1061. 
[37] H. Furuyama, Y. Koga, K. Hamasaki, F. Kuroki, N. Itami, Y. Ishikawa, Effective treatment of cyclic thrombocytopenia with cepharanthin, Pediatrics International (1999), 41, 584-585.

[38] L. Glass, M. C. Mackey, From Clocks to Chaos: The Rhythms of Life, Princeton University Press, 1988.

[39] R. S. Go, Ideopathic cyclic thrombocytopenia, Blood Reviews (2005), 19, 53-59.

[40] N. Hayes, Roots of the transcendental equation associated with a certain difference-differential equation, J. London Math. Soc. (1950), 25, 226-232.

[41] J. K. Hale, S. M. Verduyn Lunel, Introduction to Functional Differential Equations, Springer-Verlag, 1993.

[42] L. A. Harker, L. K. Roskos, U. M. Marzec, R. A. Carter, J. K. Cherry, B. Sundell, E. N. Cheung, D. Terry, W. Sheridan, Effects of megakaryocyte growth and development factor on platelet production, platelet lifespan, and platelet function in normal human volunteers, Blood (2000), 95, 2514-2529.

[43] C. Haurie, D. C. Dale, R. Rudnicki, M. C. Mackey, Modeling complex neutrophil dynamics in the grey collie, J. Theor. Biol. (2000), 204, 505-519.

[44] C. Haurie, D. C. Dale, M.C. Mackey, Cyclical neutropenia and other periodic dynamical diseases: a review of mechanisms and mathematical models, Blood (1998), 92, 2629-2640.

[45] T. Hearn, C. Haurie, M. C. Mackey, Cyclical neutropenia and the pheripheral control of white blood cell production, J. Theor. Biol. (1998), 192, 167-181.

[46] C. Helleberg, Cyclical thrombocytopenia succesfully treated with with low dose hormonal contraception, Am. J. Hematol. (1995), 48, $62-63$.

[47] R. Hoffman, R. Briddell, K. van Besien, E. Srour, T. Guscar, N. Hudson, A. Ganser, Acquired cyclical amegakaryocytic thrombocytopenia associated with an immunoglabulin blocking the action of granulocytemacrophage colonystimulating factor, N. Engl. J. Med. (1989), 312, 97-102.

[48] J. H. Horne, S. L. Baliunas, A prescription for period analysis of unevenly sampled time series, Astrophys. J. (1986), 302, 757-763.

[49] K. Kaushansky, Thrombopoietin: a tool for understanding thrombopoiesis, J. Thromb. Haemost. (2003), 1, 1578-1592. 
[50] K. Kaushansky, The molecular mechanisms that control thrombopoiesis, J. Clin. Invest. (2005), 115, 12, 3339-3347.

[51] F. Kimura, Y. Nakamura, K. Sato, N. Wakimoto, Cyclic change of cytokines in a patient with cyclical thrombocytopenia, Brit. J. Haematol. (1996), 94, 171-174.

[52] S. Kirkpatrick, C. D. Gelatt, M. P. Vecchi, Optimization by Simulated Annealing, Science (1983), 220, 4598, 671-680.

[53] V. B. Kolmanovskii, V. R. Nosov, Stability of functional differential equations, London, Orlando, Academic Press, 1986.

[54] K. Kojima, N. Fuji, E. Omoto, S. Nose, M. Yoneyama, Y. Sigii, H. Hiramatsu, M. Chikatsume, M. Satro, S. Takata, T. Itoshima, M. Tanimoto, Cyclic thrombocytopenia and polycythemia vera, Ann. Hematol. (2003), 82, 61-63.

[55] S. Kosugi, Y. Kurata, Y. Tomiyama, T. Tahara, T. Kato, S. Tadokoro, M. Shiraga, S. Honda, Y. Kanakura, Y. Matsuzawa, Circulating thrombopoietin level in chronic immune thrombocytopenic purpura, Brit. J. Haematol. (1996), 93, 704-706.

[56] S. Kosugi, Y. Tomiyama, M. Shiraga, H. Kashiwagi, H. Nakao, Y. Kanayama, Y. Kurata, Y. Matsuzawa, Cyclic thrombocytopenia associated with IgM antiGPIIb-IIa autoantibodies, Brit. J. Haematol. (1994), 88, 809-815.

[57] Y. Kuang, Delay Differential Equations with Applications in Population Dynamics, Academic Press, San Diego, 1993.

[58] R. Kashyap, V. P. Choudhry, H. P. Pati, Danazol Therapy in Cyclic Acquired Amegakaryocytic Thrombocytopenic Purpura: A Case Report, Am. J. Hematol. (1999), 60, 225-228.

[59] J. Kuyama, H. Take, S. Matsumoto, et al., Synchronous fluctuation of interleukin- 6 and platelet count in cyclic thrombocytopenia and thrombocytopoiesis, Internal Med. (1995), 34, 636-639.

[60] D. J. Kuter, R. D. Rosenberg, The reciprocal relationship of thrombopoietin (c-Mpl ligand) to changes in the platelet mass during busulfan-induced thrombocytopenia in the rabbit, Blood (1995), 85, 2720-2730.

[61] D. J. Kuter, The physiology of platelet production, Stem Cells (1996), 14, 88-101.

[62] D. J. Kuter, S. Hunt, D. Zucker-Franklin, Thrombopoiesis and thrombopoietins, Totowa, New Jersey: Humana Press, 1997. 
[63] L. G. Lajtha, R. Oliver, C. W. Gurney, Model of a bone marrow stem cell population, Brit. J. Haematol. (1962), 8, 442-460.

[64] M. L. Lewis, Cyclical Thrombocytopenia: A Thrombopoietin deficiency? J. Clin. Path. (1974), 27, 242-246.

[65] N. R. Lomb, Least-squares frequency analysis of unequally spaced data, Astrophys. Space Sci, 39, 447-462.

[66] M. C. Mackey, A unified hypothesis for the origin of aplastic anemia and periodic haematopoiesis, Blood (1978), 51, 941-956.

[67] M. C. Mackey, Periodic auto-immune hemolytic anemia: an induced dynamical disease, Bull. Math. Biol. (1979) 41, 829-834.

[68] M. C. Mackey, Cell kinetic status of hematopoietic stem cells, Cell Prolif. (2001), $34,71-83$.

[69] J. M. Mahaffy, J. Bélair, M. C. Mackey, Hematopoietic model with moving boundary condition and state dependent delay: applications in erythropoiesis, J. Theor. Biol. (1998), 190, 135-146.

[70] J. E. Menitove, J. Pereira, R. Hoffman, T. Anderson, W. Fried, R. H. Aster, Cyclical Thrombocytopenia of Apparent Autoimmune Etiology, Blood (1989), 73, 1561-1569.

[71] A. Morley, A platelet cycle in normal individuals, Aust. Ann. Med. (1969), 18, 127-129.

[72] G. S. Minot, Pupura hemorragica with lymphocytosis: an acute type and an interminent menstrual type, Am. J. Med. Sci (1936), 192, 445-456.

[73] H. Y. Mukai, H. Kojima, K. Todokoro, T. Tahara, T. Kato, Y. Hasegawa, T. Kobayashi, H. Ninomiya, T. Nagasawa, T. Abe, Serum thrombopoietin (TPO) levels in patients with amegakaryocytic thrombocytopenia are much higher than those with immune thrombocytopenic purpura, Thrombosis and Hemostasis (1996), 76, 675-678.

[74] T. Nagasawa, Y. Hasegawa, M. Kamoshita, et al., Serum thrombopoietin level is mainly regulated by megakaryocyte mass rather than platelet mass in human subjects, Brit. J. Haematol. (1998), 101, 242-244.

[75] R. M. Neal, Slice Sampling, The Annals of Statistics (2003), 31, 3, 705-767. 
[76] H. Oh, H. Nakamura, A. Yokota, T. Asai, Y. Saito, Serum thrombopoietin levels in cyclic thrombocytopenia, Blood (1996), 87, 4918.

[77] S. R. Patel, J. H. Hartwig, J. E. Italiano Jr., The biogenesis of platelets from megakaryocyte proplatelets, The Journal of Clinical Investigation (2005), 115, $12,3348-3354$.

[78] W. H. Press, B. P. Flannery, W. T. Vetterling, S. A. Teukolsky, Numerical recipes in C, 2nd edition, Cambridge University Press, 1993.

[79] S. Pavord, M. Sivakumaran, P. Furber, V. Mitchell, Cyclical thrombocyopenia as a rare manifestation of myelodysplastic syndrome, Clin. Lab. Haem. (1996), $18,221-223$.

[80] L. Pujo-Menjouet, S. Bernard, M. C. Mackey, Long period oscillations in a G0 model of hematopoietic stem cells, SIAM J. Appl. Dynam. Sys. (2005), 4(2), 312-332.

[81] L. Rice, J. L. Nichol, R. McMillan, L. K. Roskos, M. Bacile, Cyclic Immune Thrombocytopenia Responsive to Thrombopoietic Factor Therapy, Am. J. Hematol. (2001), 68, 210-214.

[82] R. Rocha, L. Horstman, Y. S. Ahn, R. Mylvaganam, W. J. Harrington, Danazol therapy for cyclic thrombocytopenia, Am. J. Hematol. (1991), 36, 140-143.

[83] M. Santillán, J. Bélair, J. M. Mahaffy, M. C. Mackey, Regulation of platelet production: The normal response to perturbation and cyclical platelet disease, J. Theor. Biol. (2000), 206, 585-603.

[84] P. Salamon, P. Sibani, R. Frost, Facts, Conjectures, and Improvemnets for Simulated Annealing, SIAM, 2002.

[85] J. D. Scargle, Studies in astronomical time series analysis, II. Statistical aspects of spectral anlysis of unevenly spaced data, Astrophys. J. (1982), 263, 835-853.

[86] T. Shirota, H. Yamamoto, H. Fujimoto, Y. Harada, K. Okada, H. Uchida, H. Roppongi, T. Hayashi, Cyclic Thrombocytopenia in a Patient Treated With Cyclosporine for Refractory Idiopathic Thrombocytopenic Purpura, Am. J. Hematol. (1997), 56, 272-276.

[87] R. Shivdasani, M. Rosenblatt, D. Zucker-Franklin, C. Jackson, P. Hunt, C. Saris, S. Orkin, Transcription factor NF-E2 is required for platelet formation independent of the actions of thrombopoietin/MGDF in megakaryocyte development, Cell (1995), 81, 695-704. 
[88] W. Skoog, J. S. Lawrence, W. S. Adams, A Methabolic Study of a Patient with Idiopatic Cyclical Thrombocytopenic Purpura, Blood (1957), 12, 844-856.

[89] R. Stoffel, A. Wiestner, R. C. Skoda, Thrombopoietin in thrombocytopenic mice: evidence against regulation at the mRNA level and for a direct regulatory role of platelets, Blood (1996), 87, 567-573.

[90] G. Stépán, Retarded Dynamical Systems: stability and characteristic functions, Longman Scientific \& Technical, 1989.

[91] J. Swinburne, M. C. Mackey, Cyclical thrombocytopenia: Characterisation by spectral analysis and a review, J. Theor. Med. (2000), 2, 81-91.

[92] G. K. von Schulthness, U. Gessner, Oscillating platelet counts in healthy individuals: Experimental investigation and quantitative evaluation of thrombocytopenic feedback control, Scand. J. Haematol. (1986), 36, 473-479.

[93] B. Telek , A. Kiss, K. Pecze, P. Ujhelyi, K. Rak, Cyclic idiopathic pure acquired amegakaryocytic thrombocytopenic purpura: a patient treated with cyclosporin A, Brit. J. Haematol. (1989), 73, 128-129.

[94] A. Tomer, A. D. Schreiber, R. McMillan, D. B. Cines, S. A. Burnstein, A. R. Thiessen, L. A. Harker, Menstrual cyclic thrombocytopenia, Brit. J. Haematol. (1989), 71, 519-524.

[95] P. Wahlberg, D. Nyman, P. Ekelund, S.A. Carlsson, H. Granlund, Cyclical thrombocytopenia with remission during lynestrenol treatment in a woman, Ann. Clin. Res. (1977), 9, 356-358.

[96] C. Wasastjerna, Cyclical Thrombocytopenia of acute type, Scand. J. Haemat. (1967), 4, 380-384.

[97] S. J. Wong, J. A. Thomas, Cytoplasmic, nuclear, and platelet autoantibodies in human granulocytic ehrlichiosis patients, J. Clin. Microbiol. (1998), 36, 19591963.

[98] T. Wilkinson, B. Firkin, Ideopathic cyclical acute thrombocytopenic purpura, Med. J. Aust. (1966), 1, 217-219.

[99] M. Yanabu, S. Nomura, T. Kukuroi, et al., Periodic production of antiplatelet autoantibody directed against GPIIIa in cyclic thrombocytopenia, Acta Haematol. (1993), 89, 155-159. 
[100] C. S. Zent, J. Ratajczak, M. Z. Ratajczak, J. Anastasi, P. C. Hoffman, A. M. Gewirtz, Relationship between megakaryocyte mass and serum thrombopoietin levels as revealed by a case of cyclic amegakaryocytic thrombocytopenic purpura, Brit. J. Haematol. (1999), 105, 452-458. 